\title{
Synthesis of Nanoparticulate Zirconia by Mechanochemical Processing
}

\author{
Aaron Dodd B.Eng.
}

School of Mechanical and Materials Engineering University of Western Australia 35 Stirling Highway, Crawley, WA 6907 Australia

This thesis is presented for the degree of Doctor of Philosophy of the University of Western Australia

October, 2002 


\section{Abstract}

This thesis presents the results of an investigation into the synthesis of ultrafine zirconia powders by mechanochemical processing. The purpose of this investigation was to identify different reaction mechanisms and to establish the effects of process variables on the properties of the final zirconia powders, specifically the average particle size and phase content.

All of the reaction systems that were investigated in this study involved reaction of anhydrous chloride precursors with an exchange reagent. However, despite this fundamental similarity, the systems were found to exhibit significantly different reaction kinetics. Depending on the exchange reagent used and the presence of inert diluent, chemical reaction of the precursors either occurred gradually during milling, by mechanically activated combustion, or only during post-milling heat-treatment.

Reaction of the $\mathrm{ZrCl}_{4}+2 \mathrm{MgO}$ system occurred gradually during milling and resulted in the formation of ultrafine $\mathrm{ZrO}_{2}$ particles embedded within a matrix of $\mathrm{MgCl}_{2}$. This allowed ultrafine $\mathrm{ZrO}_{2}$ powders to be synthesised by either milling the precursors until complete reaction was achieved or by milling for a short duration and then driving the reaction to completion by low temperature heat treatment.

The average particle size of the $\mathrm{ZrO}_{2}$ product formed by reaction of $\mathrm{ZrCl}_{4}+2 \mathrm{MgO}$ was found to decrease with milling time down to a lower limit. In contrast, the addition of $\mathrm{MgCl}_{2}$ diluent to the reactant mixture was found not to have any significant effect on the average particle size. The effect of milling duration and dilution is consistent with previous studies that suggest the average particle size is determined by the effective reaction volume.

Reactant mixtures that used $\mathrm{Li}_{2} \mathrm{O}$ as the oxide exchange did not undergo chemical reaction during milling. Milling merely resulted in amorphisation of the $\mathrm{ZrCl}_{4}$ and overall microstructural refinement. Chemical reaction, with the consequent formation of $\mathrm{ZrO}_{2}$ and $\mathrm{LiCl}$, only occurred during post-milling heat-treatment. The mechanism of 
chemical reaction during heat-treatment was found to depend on the applied heating rate and also on the presence of $\mathrm{LiCl}$ diluent in the reactant mixture.

The addition of $\mathrm{LiCl}$ diluent to the $\mathrm{Li}_{2} \mathrm{O}$ reaction systems was found to decrease the average crystallite size and increase the proportion of single crystal particles in the final $\mathrm{ZrO}_{2}$ product. For undiluted reactant mixtures, the average particle and crystallite size was found to be independent of the milling duration. In contrast, for diluted reactant mixtures, the average particle and crystallite size decreased with milling time down to a lower limit. The effect of milling duration and dilution is attributable to the high enthalpy change associated with formation of the reaction products, which allowed particle growth to occur during reaction.

The decrease in average particle size with dilution was accompanied by an increase in the tetragonal volume fraction, which is consistent with the view that the stability of the tetragonal phase in nanoparticulate $\mathrm{ZrO}_{2}$ is due to the lower specific surface energy of the tetragonal phase compared to the monoclinic.

Reaction of the $\mathrm{ZrCl}_{4}+4 \mathrm{LiOH}$ system occurred in a combustive manner following approximately 1 minute of milling and resulted in the formation of highly agglomerated $\mathrm{ZrO}_{2}$ particles. However, dilution of the reactant mixture with $4 \mathrm{LiCl}$ suppressed combustive reaction and reaction during milling resulted in the formation of a hydrated gels with an amorphous structure. This allowed $\mathrm{ZrO}_{2}$ particles to be synthesised either by milling until complete reaction or by combining a short milling time with subsequent low temperature heat treatment.

The average particle size and degree of agglomeration of the $\mathrm{ZrO}_{2}$ formed by the diluted $\mathrm{LiOH}$ reaction system was found to show a strong dependence on the milling duration. The average particle and crystallite size initially decreased with milling as a consequence of increased microstructural refinement of the reactants. Further milling beyond this initial stage resulted in aggregation of the particles before the attainment of a steady-state particle size due to the competing processes of particle aggregation and fracture. 


\section{Acknowledgements}

First of all, I would like to express my sincere thanks to Professor Paul McCormick for giving me the opportunity to undertake this work. The guidance and support that he provided during the course of my candidature is also greatly appreciated.

A great debt of gratitude is owed to my family for their support and encouragement. Without their generosity I would not have been able to complete this work. Thank you Ian, Fiona, Sarah and Jennifer.

I would like to thank the staff and post-graduate students of the School of Mechanical and Materials Engineering and SRCAMMP who provided much needed help and made my $\mathrm{PhD}$ candidature a worthwhile experience. In particular, thanks are due to Sean Galvin, Dr. Wenge Liu, Dr. Takuya Tsuzuki, Geraldine Tan, Sue Irving, Lara Cukrov, Kellie Pethick, James Hos, Dr. Teng San Ong, and Nigel Laxton.

Many thanks are due to the staff of the Centre for Microscopy and Microanalysis at the University of Western Australia. I would particularly like to thank Dr. Andrew Johnson and Steve Parry for their time and patience.

In conclusion, I would like to acknowledge the financial support provided by the School of Mechanical and Materials Engineering in the form of an Ad Hoc Postgraduate Scholarship and a departmental travel grant. 


\section{Table of Contents}

$\begin{array}{lr}\text { Abstract } & 1 \\ \text { Acknowledgements } & 3 \\ \text { Table Contents } & 4 \\ \text { List of Figures } & 8\end{array}$

Chapter 1 - Introduction

$\begin{array}{ll}\text { (1.1) Problem of Agglomeration } & 14\end{array}$

(1.2) Techniques for Synthesising Ultrafine Powders 15

(1.3) Study Overview and Objectives 16

Chapter 2 - Mechanochemical Processing

$\begin{array}{ll}\text { (2.1) Introduction } & 18\end{array}$

(2.1.1) Process Description $\quad 18$

(2.1.2) Milling Systems 19

(2.1.3) Powder Contamination 20

(2.2) Reaction Kinetics 21

(2.2.1) Introduction $\quad 21$

(2.2.2) Gradual Reaction $\quad 21$

(2.2.3) Combustive Reaction $\quad 21$

(2.2.4) Mechanically Activated Reaction 25

(2.3) Ultrafine Powder Synthesis 26

(2.3.1) Introduction $\quad 26$

(2.3.2) Reduction Reaction Systems 27

(2.3.3) Exchange Reaction Systems $\quad 29$

(2.3.4) Mechanical Grinding Systems $\quad 32$ 
(2.3.5) Agglomeration 33

(2.3.6) Particle and Crystallite Size 34

(2.3.7) Particle Morphology 35

$\begin{array}{ll}\text { Chapter 3-Zirconia } & 38\end{array}$

(3.1) Introduction $\quad 38$

(3.1.1) Engineering Applications $\quad 38$

(3.1.2) Mineral Sources and Refining $\quad 39$

$\begin{array}{ll}\text { (3.2) Crystallography } & 40\end{array}$

(3.3) Stabilised Zirconia Systems

(3.3.1) Partially Stabilised Zirconia $\quad 42$

(3.3.2) Tetragonal Zirconia Polycrystal 43

(3.4) Particle Size Stabilisation 43

(3.4.1) Kinetic Factors 45

(3.4.2) Surface Energy Effects $\quad 45$

Chapter 4 - Experimental Techniques $\quad 48$

(4.1) Powder Synthesis $\quad 48$

(4.1.1) Reagent Storage and Handling 48

(4.1.2) Mechanochemical Processing $\quad 48$

(4.1.3) Heat Treatment 49

(4.1.4) Washing and Drying $\quad 49$

(4.2) Characterisation Techniques 49

(4.2.1) X-Ray Diffraction 49

(4.2.2) Thermal Analysis 51

(4.2.3) Electron Microscopy 51

(4.2.4) Specific Surface Area Measurement 52

(4.2.5) Chemical Analysis $\quad 52$ 
(5.1) Introduction $\quad 53$

(5.1.1) Overview of Reaction Systems 53

(5.1.2) Powder Contamination $\quad 54$

(5.2) Magnesium Oxide Reactions $\quad 54$

(5.2.1) Reaction Behaviour $\quad 54$

(5.2.2) Agglomeration Behaviour $\quad 59$

(5.2.3) Effect of Dilution and Milling Duration 61

(5.2.4) Effect of Heat Treatment 63

(5.3) Lithium Oxide Reactions $\quad 66$

(5.3.1) $\mathrm{ZrO}_{2}$ Synthesis $\quad 66$

(5.3.2) Synthesis of Stabilised Zirconia 71

(5.3.3) Effect of Dilution and Milling Duration $\quad 74$

(5.3.4) Effect of Heat Treatment 78

(5.4) Lithium Hydroxide Reactions $\quad 80$

(5.4.1) $\mathrm{ZrO}_{2}$ Synthesis $\quad 80$

(5.4.2) Synthesis of Stabilised Zirconia $\quad 84$

(5.4.3) Effect of Milling Duration $\quad 88$

$\begin{array}{ll}\text { Chapter 6 - Discussion } & 95\end{array}$

$\begin{array}{ll}\text { (6.1) Introduction } & 95\end{array}$

(6.2) Reaction Mechanisms 95

(6.2.1) Adiabatic Reaction Temperature 96

(6.2.2) Magnesium Oxide Reaction 98

(6.2.3) Lithium Oxide Reactions 98

(6.2.4) Lithium Hydroxide Reactions 100 
$\begin{array}{ll}\text { (6.4) Particle and Crystallite Size } & 104\end{array}$

(6.4.1) Magnesium Oxide Reactions 104

(6.4.2) Lithium Oxide Reactions 105

$\begin{array}{ll}\text { (6.4.3) Lithium Hydroxide Reactions } & 107\end{array}$

$\begin{array}{ll}\text { (6.5) Particle Morphology } & 108\end{array}$

(6.6) Phase Formation and Stability 109

(6.5.1) Magnesium Oxide Reactions 109

(6.5.2) Lithium Oxide Reactions $\quad 110$

(6.5.3) Lithium Hydroxide Reactions $\quad 112$

Chapter 7 - Conclusions and Suggestions for Further Work 113

$\begin{array}{ll}\text { (7.1) Summary and Conclusions } & 113\end{array}$

$\begin{array}{ll}\text { (7.2) Suggestions for Further Work } & 115\end{array}$

$\begin{array}{lr}\text { References } & 117\end{array}$

$\begin{array}{lr}\text { Publications During PhD Candidature } & 129\end{array}$ 


\section{List of Figures}

Page

Figure 2.1 Vial temperature as a function of time during milling of a 22 combustive reaction system.

Figure 2.2 Schematic illustration showing the variation in the critical 22 ignition temperature for (a) gradual reaction and (b) combustion.

Figure 2.3 Schematic illustration of a growing crystal demonstrating the 37 disappearance of fast growing faces.

Figure 3.1 Schematic illustration of the low pressure polymorphs of pure $\mathrm{ZrO}_{2}$. Closed circles represent $\mathrm{Zr}^{4+}$ ions and open circles represent $\mathrm{O}^{2-}$ ions.

Figure 3.2 Equilibrium phase diagram for the $\mathrm{MgO}-\mathrm{ZrO}_{2}$ system.

Figure 3.3 Equilibrium phase diagram for the $\mathrm{Y}_{2} \mathrm{O}_{3}-\mathrm{ZrO}_{2}$ system.

Figure 5.1 XRD pattern of $\mathrm{ZrCl}_{4}+2 \mathrm{MgO}$ following (a) milling for 6 hours,

(b) washing, and (c) calcination at $500^{\circ} \mathrm{C}$ for 1 hour.

Figure 5.2 Simultaneous TG-DTA traces of milled and washed $\mathrm{ZrCl}_{4}+$ 56 $2 \mathrm{MgO}$.

Figure 5.3 $\mathrm{XRD}$ pattern of $\mathrm{ZrCl}_{4}+2 \mathrm{MgO}$ following (a) milling for 6 hours,

(b) heat-treatment at $500^{\circ} \mathrm{C}$ for 1 hour under a sealed argon atmosphere, and (c) washing.

Figure 5.4 Simultaneous TG-DTA traces of milled, heat-treated and washed 58 $\mathrm{ZrCl}_{4}+2 \mathrm{MgO}$. 
Figure 5.5 Bright field TEM images of milled $\mathrm{ZrCl}_{4}+2 \mathrm{MgO}$ following (a) $\quad 60$ washing, (b) heat-treatment and washing, (c) washing and methanol rinsing, and (d) heat-treatment, washing, and methanol rinsing.

Figure 5.6 XRD patterns of $\mathrm{ZrO}_{2}$ synthesised from $\mathrm{ZrCl}_{4}+2 \mathrm{MgO}$ using 61 milling times ranging from 10 minutes to 3 hours.

Figure 5.7 $\mathrm{XRD}$ and BET crystallite size as functions of milling time for $\mathrm{ZrCl}_{4}+2 \mathrm{MgO}$.

Figure 5.8 Variation in BET and XRD crystallite size as functions of 63 diluent content.

Figure 5.9 BET surface area of washed $\mathrm{ZrO}_{2}$ powder as a function of post64 milling heat-treatment temperature and duration.

Figure 5.10 $\mathrm{XRD}$ crystallite size of washed $\mathrm{ZrO}_{2}$ powder as a function of 64 post-milling heat-treatment temperature and duration.

Figure 5.11 Bright field TEM images of washed $\mathrm{ZrO}_{2}$ prepared from milled 65 $\mathrm{ZrCl}_{4}+2 \mathrm{MgO}$ using a post-milling heat-treatment of $720^{\circ} \mathrm{C}$ for (a) 30 minutes, (b) 1 hour, and (c) 4 hours.

Figure 5.12 XRD patterns of $\mathrm{ZrCl}_{4}+2 \mathrm{Li}_{2} \mathrm{O}+\mathrm{xLiCl}$ reactant mixtures 67 following milling for 6 hours.

Figure 5.13 DTA traces of milled $\mathrm{ZrCl}_{4}+2 \mathrm{Li}_{2} \mathrm{O}$ reactant mixtures using 68 heating rates of 20 and $80^{\circ} \mathrm{C} /$ minute. 
Figure 5.14 XRD patterns of milled $\mathrm{ZrCl}_{4}+2 \mathrm{Li}_{2} \mathrm{O}$ following heat-treatment 69 at various temperatures in the DTA using a heating rate of $20^{\circ} \mathrm{C} /$ minute.

Figure 5.15 XRD pattern of $\mathrm{ZrCl}_{4}+2 \mathrm{Li}_{2} \mathrm{O}$ following (a) milling for 6 hours, 69 (b) heat-treatment at $400^{\circ} \mathrm{C}$ using a rapid heating ramp and a hold of 1 hour, and (c) subsequent washing.

Figure 5.16 DTA traces of milled $\mathrm{ZrCl}_{4}+2 \mathrm{Li}_{2} \mathrm{O}+\mathrm{xLiCl}$ reactant mixtures 70 using a heating rate of $80^{\circ} \mathrm{C} /$ minute.

Figure 5.17 XRD patterns of washed $\mathrm{ZrO}_{2}$ synthesised from $\mathrm{ZrCl}_{4}+2 \mathrm{Li}_{2} \mathrm{O}+$ 71 $\mathrm{xLiCl}$ reactant mixtures.

Figure 5.18 DTA traces of milled reactant mixtures for the synthesis of $\mathrm{ZrO}_{2}$, 73 Y-TZP, and Mg-PSZ.

Figure 5.19 XRD patterns showing the $(101)_{\mathrm{t}}$ diffraction peak of $\mathrm{ZrO}_{2}, \mathrm{Y}$ 73 TZP, and Mg-PSZ powders that were synthesised from reactant mixtures containing $6 \mathrm{LiCl}$ diluent.

Figure 5.20 XRD and BET crystallite size as functions of milling time for 74 $\mathrm{ZrO}_{2}$ synthesised from $\mathrm{ZrCl}_{4}+2 \mathrm{Li}_{2} \mathrm{O}+6 \mathrm{LiCl}$.

Figure 5.21 Bright field TEM images of washed $\mathrm{ZrO}_{2}$ powders synthesised 75 from $\mathrm{ZrCl}_{4}+2 \mathrm{Li}_{2} \mathrm{O}+6 \mathrm{LiCl}$ using milling times (a) 30 minutes and (b) 6 hours. 
Figure 5.22 BET particle size $(\bullet)$, monoclinic crystallite size $(\diamond)$, tetragonal 76 crystallite size (ם), and tetragonal volume fraction (ם) as functions of diluent content for (a) $\mathrm{ZrO}_{2}$, (b) $\mathrm{Mg}$-PSZ, and (c) $\mathrm{Y}$ TZP.

Figure 5.23 Bright field TEM images of washed $\mathrm{ZrO}_{2}$ powders synthesised from reactant mixtures containing (a) no diluent and (b) $4 \mathrm{LiCl}$.

Figure 5.24 Specific surface area as a function of heat-treatment duration for 78 washed $\mathrm{ZrO}_{2}, \mathrm{Y}$-TZP, and Mg-PSZ powders.

Figure 5.25 Monoclinic crystallite size as a function of heat-treatment 79 duration for washed $\mathrm{ZrO}_{2}, \mathrm{Y}-\mathrm{TZP}$, and Mg-PSZ powders.

Figure 5.26 Bright field TEM images of washed $\mathrm{ZrO}_{2}$ powders synthesised 80 using post-reaction heat-treatment of $700^{\circ} \mathrm{C}$ for (a) 30 minutes, (b) 1 hour, (c) 2 hours, and (d) 4 hours.

Figure 5.27 Vial temperature as a function of time during milling of $\mathrm{ZrCl}_{4}+$ 81 $4 \mathrm{LiOH}$.

Figure 5.28 XRD pattern of $\mathrm{ZrCl}_{4}+4 \mathrm{LiOH}$ following (a) milling for 1 81 minute and (b) washing.

Figure 5.29 XRD pattern of $\mathrm{ZrCl}_{4}+4 \mathrm{LiOH}+4 \mathrm{LiCl}$ following (a) milling for 82 6 hours, (b) washing, and (c) calcination at $600^{\circ} \mathrm{C}$ for 1 hour.

Figure 5.30 TG-DTA traces of milled $\mathrm{ZrCl}_{4}+4 \mathrm{LiOH}+4 \mathrm{LiCl}$. 
Figure 5.31 XRD pattern of $\mathrm{MgCl}_{2}+2 \mathrm{LiOH}$ following (a) milling for 6 hours, (b) methanol washing, and (c) calcination at $400^{\circ} \mathrm{C}$ for 1 hour.

Figure 5.32 TG-DTA traces of $\mathrm{MgCl}_{2}+2 \mathrm{LiOH}$ that was milled for 6 hours and washed.

Figure 5.33 XRD pattern of $\mathrm{YCl} 3+3 \mathrm{LiOH}$ following (a) milling for 6 hours, (b) washing, and (c) calcination at $600 \mathrm{oC}$ for 1 hour.

Figure 5.34 TG-DTA traces of $\mathrm{YCl}_{3}+3 \mathrm{LiOH}$ that was milled for 6 hours and washed.

Figure 5.35 XRD patterns of washed and calcined $\mathrm{ZrO}_{2}, \mathrm{Mg}-\mathrm{PSZ}$, and $\mathrm{Y}-$ TZP powders synthesised from reactant mixtures containing 4LiCl diluent.

Figure 5.36 XRD patterns of washed $\mathrm{ZrO}_{2}$ synthesised from $\mathrm{ZrCl}_{4}+4 \mathrm{LiOH}$ using milling times ranging from 1 minute to 24 hours.

Figure 5.37 Crystallite size and phase content as functions of milling time 90 for $\mathrm{ZrO}_{2}$ synthesised from $\mathrm{ZrCl}_{4}+4 \mathrm{LiOH}$

Figure 5.38 Bright field TEM images of washed $\mathrm{ZrO}_{2}$ synthesised using 90 milling times of (a) 1 minute and (b) 24 hours.

Figure 5.39 Expanded view of the crystallisation exotherms of $\mathrm{ZrCl} 4+$ 92 $4 \mathrm{LiOH}+4 \mathrm{LiCl}$ reactant mixtures that were milled for times ranging from 10 minutes to 6 hours. 
Figure 5.40 XRD patterns of $\mathrm{ZrO}_{2}$ powders synthesised from $\mathrm{ZrCl}_{4}+4 \mathrm{LiOH}$ $+4 \mathrm{LiCl}$ using milling times ranging from 10 minutes to 6 hours.

Figure 5.41 Crystallite size and phase content as functions of milling time for washed $\mathrm{ZrO}_{2}$ synthesised from $\mathrm{ZrCl}_{4}+4 \mathrm{LiOH}+4 \mathrm{LiCl}$.

Figure 5.42 Bright field TEM images of washed $\mathrm{ZrO}_{2}$ synthesised from $\mathrm{ZrCl}_{4}+4 \mathrm{LiOH}+4 \mathrm{LiCl}$ using milling times of (a) 10 minutes, (b) 30 minutes, and (c) 3 hours.

Figure 6.1 Adiabatic reaction temperature as a function of diluent content for each of the three basic $\mathrm{ZrO}_{2}$ reaction systems.

Figure 6.2 Schematic illustration showing the mechanism of hard 103 agglomerate formation in water washed $\mathrm{Zr}(\mathrm{OH})_{4}$ as proposed by Lee and Readey. Adapted from [6].

Figure 6.3 Schematic illustration showing the inhibition of hard 104 agglomerate formation in methanol rinsed $\mathrm{Zr}(\mathrm{OH})_{4}$ as proposed by Lee and Readey. Adapted from [6].

Figure 6.4 Cumulative volume fraction as a function of particle diameter for $\mathrm{ZrO}_{2}$ powder synthesised from $\mathrm{ZrCl}_{4}+2 \mathrm{Li}_{2} \mathrm{O}+4 \mathrm{LiCl}$. 


\section{Chapter 1}

\section{Introduction}

Powders characterised by an average particle size of less than $100 \mathrm{~nm}$ are currently the subject of considerable interest for use in a wide variety of technological applications due to their unusual chemical and physical properties [1]. Experimental studies have demonstrated that ultrafine powders exhibit substantially enhanced chemical, mechanical, optical, and magnetic properties, which has raised the prospect for significant advances in many fields of engineering.

Ultrafine powders are primarily of interest for use as precursors in the synthesis and processing of advanced engineering materials. Compared to conventional coarse grained powders, ultrafine powders exhibit enhanced sinterability [2] and can also be used as precursors for manufacturing sub-micron and nanocrystalline ceramics [3].

The enhanced sinterability of ultrafine powders allows fully dense materials to be manufactured at significantly lower temperatures. Aside from the obvious economic benefit associated with lower energy requirements, the use of lower sintering temperatures can also successfully alleviate commonly encountered processing difficulties, such as decomposition reactions and deleterious interfacial reactions [4].

Nanocrystalline materials, which are typically manufactured by the careful consolidation of ultrafine powders, are expected to show significantly improved mechanical properties. In particular, reducing the grain size of ceramic materials to the nanocrystalline regime may enhance grain-boundary sliding and diffusion related creep phenomena thereby allowing significant plastic deformation at comparatively low temperatures $[3,5]$.

\section{(1.1) Problem of Agglomeration}

The successful exploitation of ultrafine powder particles for their enhanced processing behaviour has in general been limited by their strong tendency to agglomerate into larger secondary particles during manufacture. Unless the agglomerates are sufficiently 
weak, such that they breakdown to their primary particles during processing, the secondary particle size will determine the overall sinter activity of the powder. As a consequence, higher sintering temperatures will be required to achieve fully dense materials [6].

Powder agglomerates are commonly classified as being either soft or hard depending on the nature of the interparticle bonding. Soft agglomerates are the result of van der Waals forces and are generally easily redispersed. Hard agglomerates, however, are the result of chemical bonding between particles and often cannot readily be broken down. The strength of these agglomerates depend on the surface properties of the particles in the powder and these properties are sensitively dependent on the powder synthesis procedures.

\section{(1.2) Techniques for Synthesising Ultrafine Powders}

Techniques for the synthesis of ultrafine powders can readily be classified according to the manner in which the final nanocrystalline particle size is achieved. Broadly speaking, formation of nanocrystalline particles can be achieved either through the breakdown of a coarse grained precursor or by the controlled nucleation and growth of particles within another phase.

The most commonly used technique for the synthesis of fine powders through the breakdown of coarse grained precursors is mechanical milling, which simply uses mechanical impacts of grinding media to induce particle fracture [7]. Although this process is technically simple and finds widespread use in mineral processing, mechanical milling is of limited use for the manufacture of high quality fine powders due to high energy requirements and the inevitable contamination of the powder charge by abrasion of the grinding media.

Chemical precipitation from aqueous solution has found widespread use in industry for the manufacture of conventional coarse grained oxide powders and has also been widely investigated for the synthesis of nanocrystalline powders [8]. However, nanocrystalline powders prepared by chemical precipitation are often characterised by high levels of hard agglomeration. The precipitated species is usually a precursor rather than an oxide 
and calcination is therefore required to obtain the final powder. Due to the high surface area of the powder, even low temperature calcination allows sufficient diffusion for the formation of hard agglomerates that cannot easily be broken down during subsequent processing steps.

Gas condensation techniques form nanocrystalline materials by evaporating atoms of the desired material into a high-vacuum chamber and then rapidly cooling them in either an inert or reactive gas. The precursor material, typically metal, is vaporised using resistance, electron beam, laser or electric arc at a temperature beyond the melting point of the material, until a sufficient rate of atomisation is achieved. Subsequent cooling results in the coalescence of the evaporated atoms into nanocrystalline powder particles [9].

Vapour phase techniques are capable of producing very high quality powders with small crystallite sizes and minimal hard agglomeration. However, in practice, the successful exploitation of vapour phase techniques is limited by the trade-off between production rate and powder quality. As there is no matrix phase present to inhibit particle contact, the use of higher production rates typically leads to an increase in average particle size and the formation of hard agglomerates.

\section{(1.3) Study Objectives}

Current techniques for the synthesis of nanoparticulate materials are limited in their ability to economically manufacture high quality powders with controlled particle size and minimal hard agglomeration. As a result there is significant interest in the development of alternative synthesis techniques.

The mechanochemical synthesis technique is expected to allow substantial control over the properties of the final washed powder. Furthermore, the direct formation of fully dispersed ultrafine particles, separated by an intervening salt matrix, suggests that agglomerate formation can more readily be avoided than is possible with other synthesis methods. 
Compared to synthesis techniques such as inert gas condensation, mechanochemical processing offers significant advantages in economic terms. The equipment required for mechanochemical processing is technically simple and readily available. Furthermore the process can readily be scaled for high production rates without loss of product quality.

This thesis presents the results of an investigation into the synthesis of ultrafine zirconia powders by mechanochemical processing. The purpose of this investigation was to identify different reaction mechanisms and also to establish the effects of process variables on the properties of the final zirconia powders, specifically the average particle size, agglomeration, and phase content. 


\section{Chapter 2}

\section{Mechanochemical Processing}

\section{(2.1) Introduction}

The kinetics of solid state chemical reactions are typically limited by the rate at which the reactant species are able to diffuse across phase boundaries and through intervening product layers. As a result, conventional solid-state synthesis techniques generally require the application of high temperatures to ensure that diffusion rates are maintained at a level sufficiently high such that chemical reaction occurs within a reasonable period of time [10].

The necessity for high temperatures in conventional solid-state processing generally limits the range and characteristics of materials that can successfully be synthesised. The temperatures required for rapid solid-state diffusion are often well in excess of that required for decomposition of the desired product phase or transformation of metastable phases to their equilibrium structure. Furthermore, high processing temperatures inevitably lead to sintering and grain growth, which typically results in the formation of coarse-grained reaction products [11].

The current interest in metastable and nanocrystalline materials has prompted considerable effort towards the development of alternative processing techniques that either reduce the required processing temperatures or eliminate the need for applied heating altogether. One technique of particular interest is mechanochemical processing, which simply entails high-energy milling of a reactant powder charge [12]. This has the effect of activating chemical reaction either during the milling process itself or during subsequent low temperature heat-treatment.

\section{(2.1.1) Process Description}

Mechanochemical processing refers to a range of techniques, which can be conveniently classified as mechanical milling, mechanical alloying, or reactive milling. Although all of these techniques are based high-energy mechanical processing, they are distinguished 
from each other by the nature of the reactant powder charge and the structural changes that occur during processing. Mechanical milling involves high-energy milling of a macroscopically homogeneous material. Mechanical alloying refers to the formation of alloys by milling elemental precursors. Reaction milling uses high-energy mechanical processing to induce chemical reactions [13].

Mechanical milling can induce a wide variety of structural transformations [13,14]. However, the technique is primarily of technological interest as a simple method of manufacturing powders of nanocrystalline and amorphous materials. The intense plastic deformation arising from the milling process results in the formation of a dislocation cell structure that eventually develops into randomly oriented nanocrystalline grains. For some systems, the increased volume fraction of grain boundaries and disorder arising from milling increases the free energy of the ordered compound, thus resulting in amorphisation [14].

The underlying mechanism of mechanical alloying and reaction milling is repeated deformation, fracture and welding of the powder charge during collisions of the grinding media. Fracture of particles exposes fresh reacting surfaces and welding generates interfaces between reactant phases across which short-range diffusion can occur, thus allowing alloying or chemical reactions to occur without kinetic constraint. Diffusion rates are also enhanced by the high concentration of lattice defects, which provide "short circuit" diffusion paths $[12,13]$.

\section{(2.1.2) Milling Systems}

The most commonly used mill in experimental studies of mechanochemical processing is the vibratory Spex $8000 \mathrm{mixer} / \mathrm{mill}$. In this mill, the reactant powder charge and grinding are contained within a cylindrical vial that undergoes rapid vibratory motion in a "figure-of-eight" trajectory. The Spex mill is highly energetic, which allows the use of short milling times. However, this type of mill is restricted in the amount of material that can be processed, which typically is less than $20 \mathrm{~g}$.

Another type of mill commonly used in studies of mechanochemical processing is the planetary mill, which allows a few hundred grams of the powder to be processed. As 
implied by the name, the milling container is rotated about two separate parallel axes in a manner analogous to the rotation of a planet around the sun. The milling action of a planetary mill is similar to that of a conventional horizontal tumbling mill. However, the velocity of the grinding media is not limited by centrifugal forces. Compared to the Spex mill, the linear velocity of the grinding balls is higher but the impact frequency is lower. As a result, planetary mills are of lower energy and require longer milling times.

Attritor mills consist of a stationary container filled with grinding balls that are stirred by impellers attached to a drive shaft. The velocity of the grinding media in attritor mills is significantly lower than that in planetary or Spex type mills and consequently the energy available for mechanochemical processing is lower. However, unlike planetary and Spex type mills, attritors are readily amenable to scale-up, which allows economical mass production of powders through mechanochemical processing [15].

\section{(2.1.3) Powder Contamination}

A major issue of concern with mechanochemical processing is contamination of the powder charge since the milling action inevitably results in abrasion of the grinding media and container. The degree and type of contamination is a function of several variables, which include the composition of the powder charge, the milling system employed, and the collision energy. However, in general, the extent of contamination from the grinding media can be limited by minimising the milling duration and ensuring that the hardness of the grinding media and container is greater than that of the powder being milled [16].

One approach that has been taken for avoiding contamination of the powder charge is to use the same material for the media and container as at least one of the components of the powder charge. However, this approach is of limited applicability given the restricted range of materials that are suitable for the construction of grinding media. Furthermore, even though contamination by foreign materials is avoided by this method, the stoichiometry of the final powder will inevitably be different from that of the starting powder charge [16]. 


\section{(2.3) Reaction Kinetics}

\section{(2.3.1) Introduction}

Experimental studies of mechanochemical reaction systems have identified three distinct reaction mechanisms. Depending on the chemical nature of the reactants, the presence of inert diluents, and the milling conditions, reaction can either occur gradually during milling, by mechanically activated combustion, or during subsequent low temperature heat treatment [16].

\section{(2.3.2) Gradual Reaction}

Chemical reaction during milling either occurs gradually or suddenly by mechanically activated combustion. Whether the reaction mechanism of a given system is gradual or combustive has been found to depend on the magnitude of the enthalpy change and the milling conditions. If the enthalpy change associated with the formation of the product phases is low then the heat generated will be insufficient to significantly influence the reaction kinetics and reaction will proceed in a gradual manner. Similarly, if the milling conditions are mild enough then impacts of the grinding media will not have sufficient energy to induce self propagating reaction [17].

Gradual mechanochemical reaction systems have generally been found to exhibit sigmoidal reaction kinetics. The reaction rate initially increases with milling due to increasing activation and microstructural refinement of the reactants. The reaction rate then reaches a maximum at an intermediate milling time before decreasing as the reaction approaches completion due to dilution of the reactants by the product phases. The rate at which a gradual reaction system approaches completion is determined by the milling conditions, specifically the collision frequency and energy [18].

\section{(2.3.3) Combustive Reaction}

High-energy mechanical processing can result in the ignition of a self-propagating combustion reaction following a critical milling duration if the reaction enthalpy is high and the milling conditions are sufficiently severe [18]. 


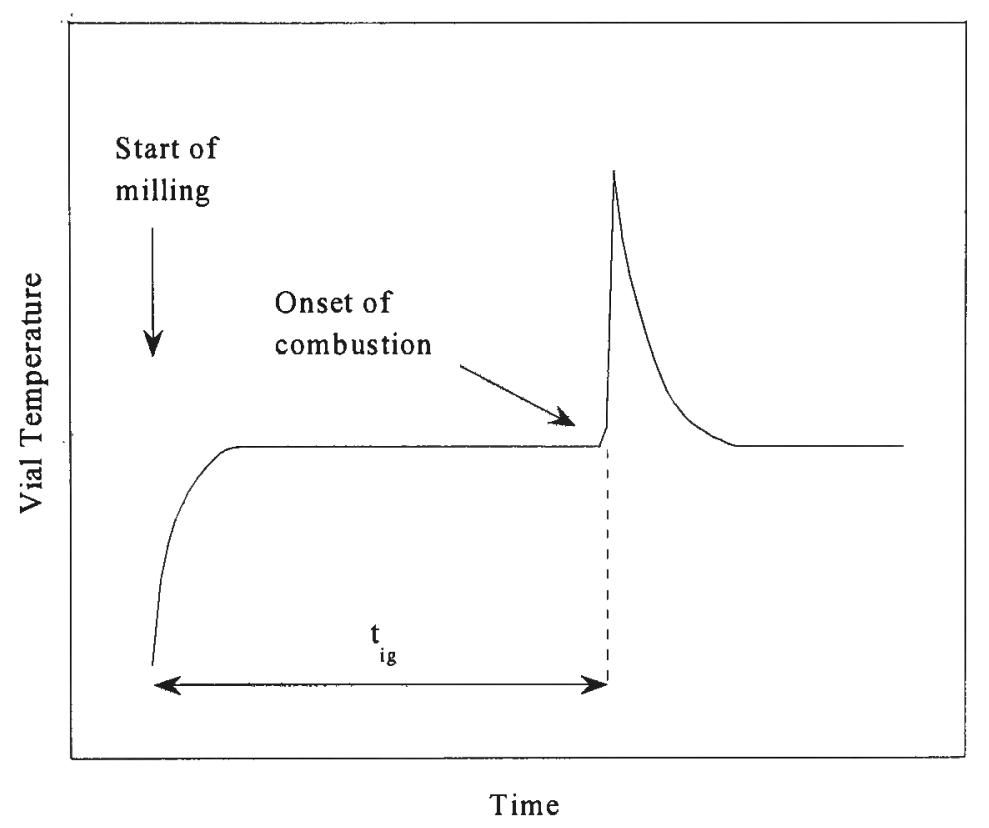

Figure 2.1 : Vial temperature as a function of time during milling of a combustive reaction system. Adapted from [19].

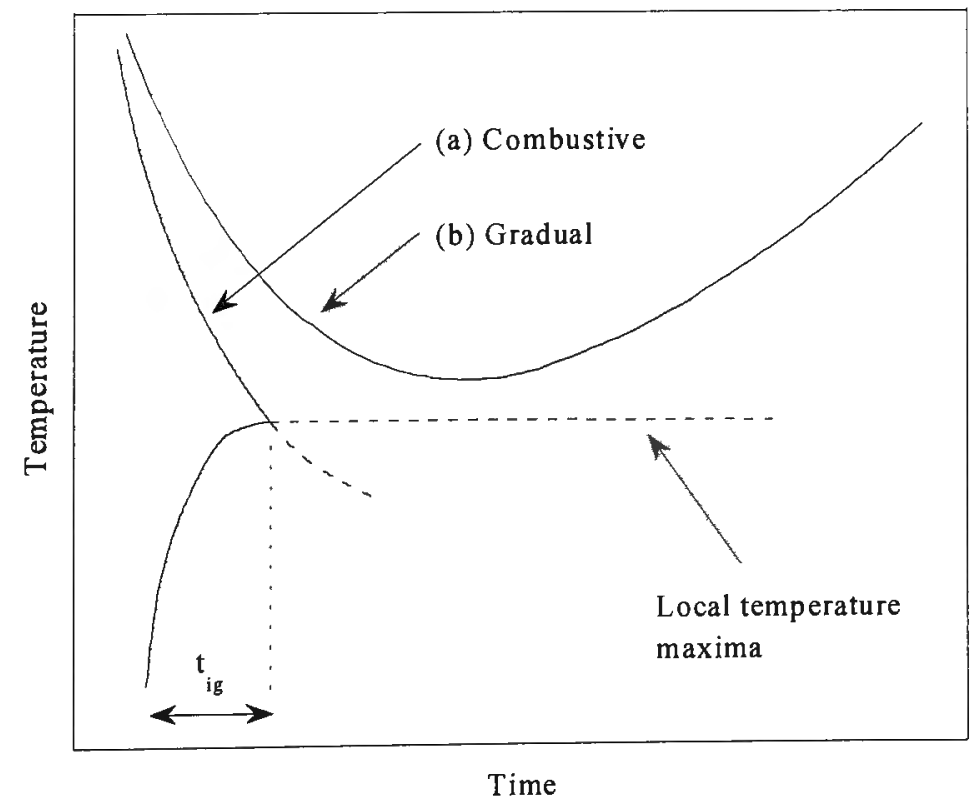

Figure 2.2 : Schematic illustration showing the variation in the critical ignition temperature for (a) gradual reaction and (b) combustion. Adapted from [19]. 
Combustion during milling is detected experimentally by the temperature spike associated with the sudden release of heat. This is illustrated in Figure 2.1, which shows a representative example of the variation in temperature of a milling vial during processing of a highly exothermic reactant mixture. In the initial stages of milling, the temperature gradually increases up to a steady-state value as a result of heat generated by collisions of the grinding media. Following the critical milling time, the vial temperature suddenly increases due to the heat generated by combustion of the reactants. The temperature then slowly decays to the previous steady-state value.

Schaffer and McCormick [19] proposed the concept of an ignition temperature as an explanation for the duration of milling required to initiate combustive reaction. In the initial stages of milling, the temperature required to ignite a self-propagating reaction is higher than the highest local temperature reached during collisions of the grinding media. However, the ignition temperature decreases with continued milling as a consequence of mechanical activation and microstructural refinement. Combustive reaction finally occurs when the ignition temperature is reduced to the highest temperature reached during collisions.

This model is illustrated schematically in Figure 2.2, which shows the variation with milling time of the required ignition temperature and the temperature reached by the powder during collisions of the grinding media. The ignition temperature initially decreases with milling time as a result of mechanical activation and microstructural refinement of the reactant powder charge. After passing through a minimum, the ignition temperature thereafter increases due to the formation of product phases that dilute the reactants. The temperature reached by the powder during collisions of the grinding media increases with milling time up to steady-state value.

The concept of an ignition temperature has successfully been used to explain the behaviour of a variety of reaction systems. For example, the expected decrease in ignition temperature with milling time has been observed experimentally for $\mathrm{ZnO}-\mathrm{Mg}$ [20] and CuO-Fe [21] reaction couples. However, studies of Mo-Si [22] and $\mathrm{V}_{2} \mathrm{O}_{5}-\mathrm{Mg}$ [23] reaction systems have shown that the model does not have universal applicability. For these two systems, it has been shown that ignition temperature does not change significantly with milling time. Takacs et al. [22] suggested that for the Mo-Si system, 
the critical milling time is the length of time required for the reactants to reach a state where thermal propagation of the reaction is possible.

Certain reduction reaction systems have been observed to exhibit what has been termed "interrupted combustion" where room temperature ageing of a pre-milled powder significantly accelerates the onset of combustion following the subsequent resumption of milling. For example, the $\mathrm{ZnO}-\mathrm{Ti}$ reaction couple was found to react in a gradual manner during milling. However, a sample that was milled for 6 hours and then aged for 12 hours underwent combustion immediately on the continuation of milling [19]. Similar behaviour has also been observed for the mechanical alloying of $\mathrm{Ni}$ and $\mathrm{Al}$ to form the NiAl intermetallic [25].

It has been suggested that diffusion of the reactant species during ageing is responsible for the interrupted combustion phenomenon. After pre-milling, the powder charge is a nanocrystalline composite of reactant phases with a high density of lattice defects, which is expected to provide favourable conditions for diffusion. Further evidence that the ageing process is the result of diffusion has also been provided by the absence of interrupted combustion in samples that have been aged at cryogenic temperature [19].

Combustion during mechanochemical processing of highly exothermic reactant mixtures can be delayed or even completely suppressed through the addition of solid or liquid diluent. For example, Schaffer and McCormick [25] found that the reduction of $\mathrm{CuO}$ with $\mathrm{Ca}$ occurred combustively within 7 minutes of milling. However, dilution of the reactant mixture with anhydrous toluene inhibited combustion and reaction then occurred in gradual manner. Similar results have also been observed for reaction systems that employ solid-state diluents.

The addition of inert diluent to an exothermic reactant mixture inhibits combustive reaction primarily by acting as an additional sink for the heat generated by chemical reaction during collision events. This has the effect of lowering the local reaction temperature. Without a sufficiently high increase in temperature the reaction is unable to undergo thermal propagation. 
Theoretical calculations of the adiabatic reaction temperature indicate that mechanochemical combustion reactions can result in significant temperature rises. For example, calculations for various metal oxide reduction reactions suggest that temperatures in the range of $3000-5000 \mathrm{~K}$ can be reached [12]. These temperature estimates represent an upper theoretical limit, as truly adiabatic conditions cannot be obtained in practice. Nevertheless experimental observations have shown that the temperatures reached in some reaction systems are sufficient to cause melting and vaporisation of the powder charge [17].

\section{(2.3.4) Mechanically Activated Reaction}

For certain reaction systems, milling does not induce chemical reaction of the precursors directly. However, as a consequence of the microstructural refinement and accumulation of lattice defects arising from the milling action, chemical reaction during subsequent thermal processing is able to occur at reduced temperatures. In these particular cases, inhibition of chemical reaction during milling arises from either unfavourable thermodynamics at the milling temperature or kinetic inhibition.

The mechanically activated reduction of $\mathrm{TiO}_{2}$ with graphite represents an example of a reaction system that does not react during milling as a consequence of the positive free energy change associated with the formation of the $\mathrm{TiC}$ and $\mathrm{CO}_{(\mathrm{g})}$ product phases at low temperatures. Ren et al. [26] found milling of $\mathrm{TiO}_{2}+3 \mathrm{C}$ merely resulted in amorphisation of the graphite reactant and overall microstructural refinement. As a consequence of milling, the temperature required for complete reaction during subsequent heat-treatment was reduced by approximately $500^{\circ} \mathrm{C}$. Similar behaviour has also been observed for mechanically activated synthesis of $\mathrm{SiC}$ by reaction of $\mathrm{SiO}_{2}+3 \mathrm{C}$ [26].

The absence of chemical reaction during milling has also been observed in a variety of systems for which the free energy change is negative. For example, reaction of various metal chlorides $\left(\mathrm{FeCl}_{3}, \mathrm{ZrCl}_{4}\right.$, and $\left.\mathrm{AlCl}_{3}\right)$ with $\mathrm{CaO}$ only occurs during post-milling heat-treatment even though reaction is thermodynamically favourable at low temperatures [27-29]. For these systems it can reasonably be supposed that there is an energy barrier to reaction that was not overcome during milling. 
The phenomenon of mechanically activated reaction has been applied to combustion synthesis in a process termed MASHS (mechanically activated self propagating high temperature synthesis). In this process, powder precursors are subjected to a brief period of comparatively mild mechanical milling and are then heated to ignite a combustion reaction. This has been used to synthesise a variety of intermetallic compounds without the use of long milling times, thus minimising contamination from the grinding media. In addition, it has been shown that mechanical activation enables weakly exothermic reactant mixture to undergo self sustaining reaction [30].

\section{(2.6) Ultrafine Powder Synthesis}

\section{(2.6.1) Introduction}

Mechanochemical processing has recently been used for the synthesis of ultrafine powders. In this process, high-energy mechanical processing of chemical precursors is used to manufacture a composite powder consisting of fully dispersed ultrafine powder particles embedded within a salt matrix. The ultrafine powder is then recovered by selective dissolution of the salt matrix through washing with an appropriate solvent.

Although numerous processes exist for the synthesis of nanoparticulate materials, the mechanochemical synthesis technique is of particular interest since it is applicable to a wide variety of materials and is capable of producing powders with minimal hard agglomeration. In contrast to other synthesis techniques where particles are able to encounter each other during processing, mechanochemical processing effectively encapsulates each individual powder particle in by-product salt, thus separating the particles and thereby minimising the potential for hard agglomerate formation.

Within the basic framework of the mechanochemical synthesis technique, formation of the composite structure consisting of ultrafine particles dispersed within a salt matrix can occur in various ways. In the majority of systems that have been investigated to date, the composite structure is developed during milling as a consequence of a chemical displacement reaction between a halide or oxide precursor and either a reductant or exchange reagent. However, several systems have also been investigated where the milling process is merely used for microstructural refinement of a reactant 
mixture and formation of the final composite structure occurs during subsequent low temperature heat-treatment.

The following sections present an overview of various mechanochemical reaction systems that have been developed for the synthesis of ultrafine powders. The overview is divided into three parts according to the type reaction the system utilises.

\section{(2.6.2) Reduction Reaction Systems}

Reaction systems based on reduction reactions have successfully been used for the synthesis of a wide range of materials, including elemental metals [31-34], amorphous alloys [35], magnetic intermetallics [36], carbide and nitride hard metals [37], and oxides [38]. As shown in Table 2.1, the majority of these systems involve reduction of metal chloride or oxide precursors with a reactive metal. However, reaction systems have also been developed for synthesising metal oxide powders by the reduction of alkali metallates with sulfur [38].

Ding et al. [31-34] investigated the synthesis of ultrafine powders of various transition metals by reduction of anhydrous chloride precursors with alkali and alkaline earth metals. These reaction systems are characterised a large enthalpy change, which indicates a strong tendency towards combustive reaction. The high temperatures associated with combustive reaction can lead to melting and vaporisation of the reaction products, which typically results in the formation of powders with a wide particles size distribution and extensive hard agglomeration. The successful exploitation of these systems therefore necessitates the addition of inert diluent and the use of mild milling conditions to ensure that chemical reaction occurs in a gradual manner.

In their study of the $\mathrm{CuCl}_{2}+2 \mathrm{Na}$ reaction system, Ding et al. [34] conducted an investigation into the effect of dilution and milling conditions on the reaction mechanism. Decreasing the ball size from 12.6 to $3.2 \mathrm{~mm}$ was found to delay the onset of combustion from 12 to $140 \mathrm{~min}$. Dilution with $100 \mathrm{wt} \%$ of $\mathrm{NaCl}$ completely inhibited combustion. 
The use of mechanochemical reduction reactions for the synthesis of ultrafine metal powders has subsequently been extended to allow the production of intermetallics and metallic alloys. For example, Liu and McCormick [36] used the co-reduction of samarium and cobalt oxides with calcium metal to manufacture ultrafine powders of $\mathrm{Sm}_{2} \mathrm{Co}_{17}$ and $\mathrm{SmCo}_{5}$ magnetic intermetallics. Similarly, Wu et al. [35] prepared amorphous $\mathrm{Cu}_{40} \mathrm{Zr}_{60}$ nanoparticles by the co-reduction of $\mathrm{CuCl}$ and $\mathrm{Zr}\left(\mathrm{SO}_{4}\right)_{2}$ with $\mathrm{Mg}$ metal.

\begin{tabular}{|c|c|c|c|c|c|}
\hline \multirow[t]{2}{*}{ Powder } & \multirow[t]{2}{*}{ Reactants } & \multirow[t]{2}{*}{ Dilution } & \multirow{2}{*}{$\begin{array}{l}\text { Heat } \\
\text { Treatment }\end{array}$} & Particle & \multirow[t]{2}{*}{ Ref. } \\
\hline & & & & Size $(n m)$ & \\
\hline \multirow[t]{3}{*}{$\mathrm{Fe}$} & $\mathrm{FeCl}_{3}+3 \mathrm{Na}$ & $50 \% \mathrm{NaCl}$ & No & $15-20 \mathrm{~nm}$ & 32 \\
\hline & $2 \mathrm{FeCl}_{3}+3 \mathrm{Ca}$ & No & No & $30-35 \mathrm{~nm}$ & 32 \\
\hline & $\mathrm{FeCl}_{3}+\mathrm{Al}$ & $100 \% \mathrm{NaCl}$ & No & $50-60 \mathrm{~nm}$ & 32 \\
\hline $\mathrm{Co}$ & $\mathrm{CoCl}_{2}+2 \mathrm{Na}$ & $50 \% \mathrm{NaCl}$ & No & $20-20 \mathrm{~nm}$ & 33 \\
\hline $\mathrm{Ni}$ & $\mathrm{NiCl}_{2}+2 \mathrm{Na}$ & $50 \% \mathrm{NaCl}$ & No & $10-20 \mathrm{~nm}$ & 31,33 \\
\hline $\mathrm{Cu}$ & $\mathrm{CuCl}_{2}+2 \mathrm{Na}$ & $50 \% \mathrm{NaCl}$ & No & $20-50 \mathrm{~nm}$ & 34 \\
\hline \multirow[t]{2}{*}{$\mathrm{Cu}_{40} \mathrm{Zr}_{60}$} & $5 \mathrm{CuCl}+12 \mathrm{Zr}\left(\mathrm{SO}_{4}\right)_{2}+$ & $15 \% \mathrm{NaCl}$ & No & $40 \mathrm{~nm}$ & 35 \\
\hline & $4 \mathrm{Mg}$ & & & & \\
\hline $\mathrm{Sm}_{2} \mathrm{Co}_{17}$ & $\mathrm{Sm}_{2} \mathrm{O}_{3}+17 \mathrm{CoO}+20 \mathrm{Ca}$ & $200 \% \mathrm{CaO}$ & $800^{\circ} \mathrm{C}$ & $10-250 \mathrm{~nm}$ & 36 \\
\hline \multirow[t]{2}{*}{$\mathrm{TiC}$} & $\mathrm{TiO}_{2}+2 \mathrm{Mg}+\mathrm{C}$ & No & $1200^{\circ} \mathrm{C}$ & $16-20 \mathrm{~nm}$ & 37 \\
\hline & $\mathrm{FeTiO}_{3}+3 \mathrm{Mg}+\mathrm{C}$ & No & $1200^{\circ} \mathrm{C}$ & $16-20 \mathrm{~nm}$ & 37 \\
\hline \multirow[t]{2}{*}{ TiN } & $2 \mathrm{TiO}_{2}+4 \mathrm{Mg}+\mathrm{N}_{2}$ & No & $1200^{\circ} \mathrm{C}$ & $16-20 \mathrm{~nm}$ & 37 \\
\hline & $2 \mathrm{FeTiO}_{3}+6 \mathrm{Mg}+\mathrm{N}_{2}$ & No & $1200^{\circ} \mathrm{C}$ & $16-20 \mathrm{~nm}$ & 37 \\
\hline $\mathrm{Cr}_{2} \mathrm{O}_{3}$ & $\mathrm{Na}_{2} \mathrm{Cr}_{2} \mathrm{O}_{7}+\mathrm{S}$ & $\mathrm{Na}_{2} \mathrm{SO}_{4}$ & $520^{\circ} \mathrm{C}$ & $10-80 \mathrm{~nm}$ & 38 \\
\hline
\end{tabular}

Table 2.1 : Summary of reduction reaction systems used for the mechanochemical synthesis of ultrafine powders.

As was observed for reaction systems based on the reduction of metal chlorides, Liu and McCormick [36] also found that the highly exothermic nature of the co-reduction of $\mathrm{Sm}_{2} \mathrm{O}_{3}$ and $\mathrm{CoO}$ required the addition of inert diluent to inhibit combustive reaction. However, it was also noted that the presence of a certain amount of diluent was necessary for mechanochemical reaction to occur at all. Without any diluent, the high 
volume fraction of ductile $\mathrm{Ca}$ metal in the reactant mixture resulted in severe cold welding, which prevented reaction.

Welham and Llewellyn [37] investigated the synthesis of $\mathrm{TiC}$ and $\mathrm{TiC}$ by the reduction of rutile $\left(\mathrm{TiO}_{2}\right)$ and ilmenite $\left(\mathrm{FeTiO}_{3}\right)$ powders by magnesium metal in the presence of either graphite or nitrogen. The by-product phases formed during milling were readily removed by a simple acid leaching process, which allowed recovery of the carbide or nitride product. Examination of the final washed powder by TEM and XRD showed that the process resulted in the formation of single crystal particles with an average particle size smaller than approximately $20 \mathrm{~nm}$.

As discussed in the following section, mechanochemical reaction systems for the synthesis of oxide powders are typically based on reaction of a metal salt precursor with an oxide exchange reagent. However, Tsuzuki and McCormick [38] have shown that suitably designed reduction reactions can also be used for the synthesis of ultrafine oxide powders. In their study, ultrafine powder particles of $\mathrm{Cr}_{2} \mathrm{O}_{3}$ were prepared by the reduction of $\mathrm{Na}_{2} \mathrm{Cr}_{2} \mathrm{O}_{7}$ with $\mathrm{S}$.

\section{(2.6.3) Exchange Reaction Systems}

Mechanochemical reaction systems that have been developed for the synthesis of compounds are generally based on reaction of either an oxide or chloride precursor with an exchange reagent. Although this basic reaction scheme is applicable to the synthesis of a wide range of materials, as demonstrated by previous work on solid-state metathesis reactions [39], only a limited variety has been synthesised to date by mechanochemical processing. As shown in Table 2.2, the majority of compounds that have been prepared to date are various oxides and sulfides.

Ding et al. investigated mechanically activated reaction of anhydrous chloride precursors with $\mathrm{CaO}$ for the synthesis of ultrafine $\mathrm{Fe}_{2} \mathrm{O}_{3}$ [27], $\mathrm{Al}_{2} \mathrm{O}_{3}$ [28], and $\mathrm{ZrO}_{2}$ [29] powders. For these systems, milling of the reactant mixtures resulted in amorphisation of the chloride precursor and overall microstructural refinement to the nanocrystalline regime. Chemical reaction, with the consequent formation of ultrafine oxide particles and $\mathrm{CaCl}_{2}$, was only found to occur during subsequent low-temperature heat-treatment. 
In addition to examining the mechanochemical reaction of $2 \mathrm{FeCl}_{3}+3 \mathrm{CaO}$, Ding et al. [27] also studied the $2 \mathrm{FeCl}_{3}+3 \mathrm{Ca}(\mathrm{OH})_{2}$ reaction system. Reaction was found to occur in a gradual manner during milling rather than only during subsequent heat-treatment, which clearly demonstrates the significant impact that incipient water can exert on the kinetics of mechanochemical reactions.

\begin{tabular}{|c|c|c|c|c|c|}
\hline Powder & Reactants & Dilution & $\begin{array}{l}\text { Heat } \\
\text { Treatment }\end{array}$ & $\begin{array}{l}\text { Particle } \\
\text { Size }(\mathrm{nm})\end{array}$ & Ref. \\
\hline $\mathrm{Al}_{2} \mathrm{O}_{3}$ & $2 \mathrm{AlCl}_{3}+3 \mathrm{CaO}$ & No & $350^{\circ} \mathrm{C}$ & $10-20 \mathrm{~nm}$ & 28 \\
\hline $\mathrm{ZrO}_{2}$ & $\mathrm{ZrCl}_{4}+2 \mathrm{CaO}$ & No & $300^{\circ} \mathrm{C}$ & $5-10 \mathrm{~nm}$ & 29 \\
\hline \multirow[t]{2}{*}{$\mathrm{Fe}_{2} \mathrm{O}_{3}$} & $2 \mathrm{FeCl}_{3}+3 \mathrm{CaO}$ & $5 \mathrm{CaCl}_{2}$ & $150^{\circ} \mathrm{C}$ & $10-30 \mathrm{~nm}$ & 27 \\
\hline & $2 \mathrm{FeCl}_{3}+3 \mathrm{Ca}(\mathrm{OH})_{2}$ & No & $200^{\circ} \mathrm{C}$ & $20-50 \mathrm{~nm}$ & 27 \\
\hline $\mathrm{MnFe}_{2} \mathrm{O}_{4}$ & $2 \mathrm{FeCl}_{3}+4 \mathrm{MnO}$ & No & $600^{\circ} \mathrm{C}$ & $9.5-40 \mathrm{~nm}$ & 40 \\
\hline \multirow[t]{2}{*}{$\mathrm{Gd}_{2} \mathrm{O}_{3}$} & $2 \mathrm{GdCl}_{3}+3 \mathrm{CaO}$ & No & $700^{\circ} \mathrm{C}$ & $100 \mathrm{~nm}$ & 41 \\
\hline & $\mathrm{GdCl}_{3}+3 \mathrm{NaOH}$ & $3 \mathrm{NaCl}$ & $500^{\circ} \mathrm{C}$ & $20 \mathrm{~nm}$ & 42 \\
\hline \multirow[t]{2}{*}{$\mathrm{BaFe}_{12} \mathrm{O}_{19}$} & 1.2 $2 \mathrm{BaCl}_{2}+12 \mathrm{FeCl}_{3}+$ & $200 \% \mathrm{NaCl}$ & $800^{\circ} \mathrm{C}$ & $10-20 \mathrm{~nm}$ & 43 \\
\hline & $38.4 \mathrm{NaOH}$ & & & & \\
\hline $\mathrm{ZnO}$ & $\mathrm{ZnCl}_{2}+\mathrm{Na}_{2} \mathrm{CO}_{3}$ & $8.6 \mathrm{NaCl}$ & $400^{\circ} \mathrm{C}$ & $10-30 \mathrm{~nm}$ & 44 \\
\hline \multirow[t]{2}{*}{$\mathrm{SnO}_{2}$} & $\mathrm{SnCl}_{2}+\mathrm{Ca}(\mathrm{OH})_{2}$ & $\mathrm{CaCl}_{2}$ & $400^{\circ} \mathrm{C}$ & $1-60 \mathrm{~nm}$ & 45 \\
\hline & $\mathrm{SnCl}_{2}+\mathrm{Na}_{2} \mathrm{CO}_{3}$ & $\mathrm{NaCl}$ & $700^{\circ} \mathrm{C}$ & $5-40 \mathrm{~nm}$ & 46 \\
\hline \multirow[t]{2}{*}{$\mathrm{CeO}_{2}$} & $\mathrm{CeCl}_{3}+3 \mathrm{NaOH}$ & $3 \mathrm{NaCl}$ & $500^{\circ} \mathrm{C}$ & $10 \mathrm{~nm}$ & 47 \\
\hline & $2 \mathrm{CeCl}_{3}+3 \mathrm{CaO}$ & No & $400^{\circ} \mathrm{C}$ & $19 \mathrm{~nm}$ & 48 \\
\hline $\mathrm{ZnS}$ & $\mathrm{ZnCl}_{2}+\mathrm{CaS}$ & $3.6 \mathrm{CaCl}_{2}$ & No & $7-9 \mathrm{~nm}$ & 49 \\
\hline $\mathrm{CdS}$ & $\mathrm{CdCl}_{2}+\mathrm{Na}_{2} \mathrm{~S}$ & $\mathrm{NaCl}$ & No & $4-8 \mathrm{~nm}$ & 50 \\
\hline $\mathrm{Ce}_{2} \mathrm{~S}_{3}$ & $2 \mathrm{CeCl}_{3}+3 \mathrm{CaS}$ & $11 \mathrm{CaCl}_{2}$ & No & $20 \mathrm{~nm}$ & 51 \\
\hline $\mathrm{GaN}$ & $\mathrm{Ga}_{2} \mathrm{O}_{3}+\mathrm{Mg}_{3} \mathrm{~N}_{2}$ & & & & 52 \\
\hline
\end{tabular}

Table 2.2 : Summary of exchange reaction systems used for the mechanochemical synthesis of ultrafine powders. 
Tsuzuki et al. [41] investigated the synthesis of rare earth oxides by mechanochemical reaction of chloride precursors with $\mathrm{CaO}$. The synthesis of rare earth oxides presents a problem since the reaction given by equation (2.1):

$$
2 \mathrm{ROCl}+\mathrm{MO} \rightarrow \mathrm{R}_{2} \mathrm{O}_{3}+\mathrm{MCl}_{2}
$$

is characterised by a positive free energy change for the lightweight lanthanides (where $\mathrm{R}$ is a rare earth and $\mathrm{M}$ is an alkaline earth metal). As a result the formation of the oxychloride phase is thermodynamically favoured over the oxide. The reaction is thermodynamically favourable only for $\mathrm{Gd}$ and the heavier lanthanides. However, despite this, the $2 \mathrm{GdCl}_{3}+3 \mathrm{CaO}$ reaction system was found to be unsuitable because formation of the desired $\mathrm{Gd}_{2} \mathrm{O}_{3}$ product only occurred during post-milling heattreatment at high temperature, which resulted in particle growth.

The thermodynamic limitations associated with exchange reactions based on alkaline earth metal oxides prompted the development of alternative reaction systems that used exchange reagents based on alkali metals. For example, Tsuzuki et al. [42] investigated the synthesis of $\mathrm{Gd}_{2} \mathrm{O}_{3}$ by reaction of $\mathrm{GdCl}_{3}+3 \mathrm{NaOH}$. For this system, reaction occurred gradually during milling and resulted in the formation of $\mathrm{Gd}(\mathrm{OH})_{3}$, which decomposed to oxide during subsequent calcination.

$\mathrm{NaOH}$ has also been used as an oxide exchange reagent in reaction systems for the synthesis of $\mathrm{BaFe}_{12} \mathrm{O}_{19}$ [43] and $\mathrm{CeO}_{2}$ [47] from chloride precursors. Both of these reaction systems are highly exothermic. As a result, dilution with $\mathrm{NaCl}$ was found to be necessary to inhibit combustive reaction and thereby allow the formation of ultrafine powder particles with a narrow size distribution.

Muroi et al. [40] used an exchange reaction between $\mathrm{MnO}$ and $\mathrm{FeCl}_{3}$ to manufacture an ultrafine powder of $\mathrm{MnFe}_{2} \mathrm{O}_{4}$. The initial reactant mixture contained an excess of the exchange reagent, which reacted with $\mathrm{Fe}_{2} \mathrm{O}_{3}$ product to form the final $\mathrm{MnFe}_{2} \mathrm{O}_{4}$ product phase. This basic reaction system outline appears to be applicable for the synthesis of a wide variety of other complex oxide phases. However, no other similar reaction systems have yet been reported in the literature. 
Aside from the oxide compounds discussed above, mechanochemical exchange reactions have also been investigated for the synthesis of sulfide compounds. Tsuzuki et al. [49-52] used exchange reactions between anhydrous chloride precursors and either $\mathrm{CaS}$ or $\mathrm{Na}_{2} \mathrm{~S}$ to synthesise ultrafine powders of $\mathrm{ZnS}, \mathrm{CdS}$, and $\mathrm{Ce}_{2} \mathrm{~S}_{3}$. All of three of these systems were found to undergo reaction during milling in a gradual manner.

\section{(2.6.4) Mechanical Grinding Systems}

In addition to the systems based on chemical displacement reactions, systems that simply utilise the microstructural refinement arising from the milling action have also been investigated. These systems simply involve milling a suitable precursor with a diluent phase. The milling action results in microstructural refinement of the precursor and disperses it within the diluent phase. Such systems are distinguished from simple mechanical grinding in that the diluent acts to prevent the formation of particle agglomerates.

Liu et al. [53]: developed a process for the synthesis of ultrafine $\alpha-\mathrm{Fe}_{2} \mathrm{O}_{3}$ powders by dispersing a precipitated hydroxide precursor in $\mathrm{NaCl}$ through high-energy ball milling. The milling process converted the amorphous hydroxide precursor into fully dispersed particles of crystalline $\alpha-\mathrm{Fe}_{2} \mathrm{O}_{3}$ with an average diameter of approximately $10 \mathrm{~nm}$. A similar process has been investigated by Shi et al $[54,55]$ for the synthesis of $\mathrm{CoFe}_{2} \mathrm{O}_{4}$ and $\mathrm{NiFe}_{2} \mathrm{O}_{4}$ spinel ferrites. Powders of the same composition that were prepared by direct calcination of the as-precipitated precursor were characterised by an agglomerate size that was significantly larger than the primary crystallite size.

Fah et al. [56] investigated the synthesis of ultrafine $\mathrm{ZnO}$ powders by high energy milling of a hydrated zinc nitrate hydroxide precursor with $\mathrm{NaCl}$. Milling resulted in the formation of dispersed $\mathrm{ZnO}$ particles with an average particle size of approximately 20 nm. Interestingly, decomposition of the precursor was only found to occur in the presence of the diluent even though the $\mathrm{NaCl}$ remained chemically inert during mechanochemical processing.

The systems investigated by Liu [53], Shi [54,55], and Fah [56] all used precursor phases that were already nanometric prior to the milling process. Although some 
microstructural refinement did occur during milling, the main effect was one of dispersal throughout the salt matrix. In contrast to these systems, Welham [57] used mechanical grinding of coarse-grained $\mathrm{Ge}(<200 \mu \mathrm{m})$ with $\mathrm{MgO}$ to synthesise ultrafine Ge powders. Following milling, Ge particles with an average crystallite size of approximately $40 \mathrm{~nm}$ were recovered by removing the $\mathrm{MgO}$ matrix phase through acid leaching.

\section{(2.6.5) Agglomeration}

Experimental studies of mechanochemical reaction systems have shown it is only possible to form powders consisting of dispersed single crystal particles where the particle volume fraction in the reaction product is sufficiently low. It is known from percolation theory of particulate composites that the long-range connectivity of particles increases dramatically with their volume fraction at the percolation threshold [58]. Therefore, if the volume fraction of particles in the reaction product is above the percolation threshold then an interconnected network will be formed rather than distinct single crystal particles.

The volume fraction of particles that corresponds to the percolation threshold is dependent on a wide variety of factors. As a result, it is not possible to define a single value for the percolation threshold that has universal applicability. However, Tsuzuki and McCormick have suggested a particle volume fraction of approximately $20 \%$ as a guideline [45], which is approximately equal to the value for site percolation of monodisperse particles with random lattice positions [58].

Mechanochemical reaction of the $\mathrm{SnCl}_{2}+\mathrm{Ca}(\mathrm{OH})_{2}$ system is representative example that clearly demonstrates the necessity of a sufficiently low particle volume fraction in the reaction product [45]. Without the addition of inert $\mathrm{CaCl}_{2}$ diluent, the volume fraction was $29 \%$ and the resultant $\mathrm{SnO}_{2}$ powder consisted of relatively large aggregates of smaller crystallites. Reducing the volume fraction of $\mathrm{SnO}_{2}$ to $9 \%$ by the addition of sufficient $\mathrm{CaCl}_{2}$ diluent resulted in the formation of dispersed particles. Similar results have also been observed in other reaction systems [27]. 


\section{(2.6.6) Particle and Crystallite Size}

The mechanochemical synthesis technique has been shown to offer considerable scope for controlling the average particle and crystallite size of the resultant powder. Any parameter that affects the crystallite size developed during milling will have a corresponding effect on the average particle size of the final washed powder. In addition, post-milling heat treatment of sufficient temperature and duration can be used to coarsen the average particle size through diffusional mass flow and interparticle sintering.

For systems that do not react during milling, it has been suggested that the crystallite size of the as-milled reactants should determine the average particle size of the resultant powder. Consistent with this expectation, Ding et al. [29] found that the average $\mathrm{ZrO}_{2}$ particle size formed during heat-treatment of milled $\mathrm{ZrCl}_{4}+2 \mathrm{CaO}$ could be related the crystallite size of the $\mathrm{CaO}$ reactant. Although not yet confirmed by a systematic experimental study, this relation indicates that varying the milling duration and intensity could be used to control the average particle size of the final washed powder.

Previous studies of mechanical grinding and alloying systems have shown that the crystallite size of the powder charge decreases with milling time down to steady-state value [14]. It could therefore reasonably be expected that the average particle size of a powder formed by mechanically activated reaction would exhibit a similar variation with milling time. The relationship between the scale of the as-milled microstructure and the final powder being given by the volumetric change associated with formation of the final product phases.

For systems that react gradually during milling, it has been found that the variation in particle size with milling time depends on the mechanical properties of the product phase. Highly ductile materials, such as elemental metals, tend to undergo growth with extended milling through interparticle welding during collision events [31]. In contrast, hard and brittle powders show minimal tendency towards aggregation during extended milling [46]. 
The effect of milling intensity has been examined in a few reaction systems by varying the size of the milling balls. Tsuzuki and McCormick [50] found that the average crystallite size of the CdS product formed by reaction of $\mathrm{CdCl}_{2}+\mathrm{Na}_{2} \mathrm{~S}$ could be reduced by decreasing the ball size. Milling of the reactants with a ball size of $12.6 \mathrm{~mm}$ resulted in an average crystallite size of $8 \mathrm{~nm}$. Reducing the ball size to $4.8 \mathrm{~mm}$ gave a powder characterised by an average crystallite size $4.3 \mathrm{~nm}$. In contrast to this behaviour, Cukrov et al. [46] found that varying the ball size yielded no significant variation in the particle size of $\mathrm{SnO}_{2}$ particles that were synthesised from the $\mathrm{SnCl}_{2}+\mathrm{Na}_{2} \mathrm{CO}_{3}$ system.

Tsuzuki and McCormick [47] found that the average particle size of $\mathrm{CeO}_{2}$ prepared by reaction of $\mathrm{CeCl}_{3}+3 \mathrm{NaOH}$ could be controlled over a wide range by varying the postmilling heat-treatment temperature. Raising the heat-treatment temperature from 500 to $800^{\circ} \mathrm{C}$ increased the average particle and crystallite size from 10 to approximately 30 $\mathrm{nm}$. Examination of the powders by TEM showed that the increase in particle size over this temperature range was accompanied by an increase in the size distribution, which suggests that growth occurred by Ostwald ripening. Particle growth through sintering of individual crystallites only occurred at temperatures above the melting point of the $\mathrm{NaCl}$ reaction by-product $\left(801^{\circ} \mathrm{C}\right)$.

\section{(2.6.6) Particle Morphology}

Anisotropic grain growth of materials in molten salt has previously found application for the synthesis of powders with specific particle morphologies [59-69]. As shown in Table 2.3, a wide variety of materials have successfully been synthesised by this technique. In this process, powder precursors are mixed together with a suitable salt and then heat-treated at a temperature above the melting point of the salt. The powder is then recovered by removing the salt with a suitable washing process. During heattreatment, the molten salt acts as a flux that increases diffusion rates, thus accelerating the kinetics of grain growth.

As discussed in previous sections, the mechanochemical synthesis technique results in the formation of a nanocrystalline composite consisting of ultrafine powder particles embedded within a salt matrix. This allows ready application of the molten salt processing technique for the synthesis of ultrafine powders with anisotropic particle 
shapes. The reacted powder is simply subjected to a heat treatment at a temperature above the melting point of the salt by-product.

In the absence of any restraint, the shape of a growing crystal is determined by the relative rate at which the various crystal faces undergo growth. The fastest growing faces eventually disappear leaving the crystal bounded by the slowest growing faces [68]. This is illustrated in Figure 2.3, which shows the growth behaviour of a hypothetical 2-dimensional particle where the crystal faces marked ' $A$ ' grow faster than those marked ' $B$ '. The faster growing ' $A$ ' faces eventually grow out, which leaves the final crystal bounded by the slow growing ' $\mathrm{B}$ ' faces.

\begin{tabular}{llllll}
\hline Material & Flux & $\begin{array}{l}\text { Temperature } \\
\left({ }^{\circ} \mathrm{C}\right)\end{array}$ & $\begin{array}{l}\text { Duration } \\
(\mathrm{hrs})\end{array}$ & $\begin{array}{l}\text { Particle } \\
\text { Morphology }\end{array}$ & Reference \\
\hline $3 \mathrm{Al}_{2} \mathrm{O}_{3} .2 \mathrm{SiO}_{2}$ & $\mathrm{~K}_{2} \mathrm{SO}_{4}$ & 1100 & 3 & Whiskers & 59 \\
$\mathrm{Bi}_{4} \mathrm{Ti}_{3} \mathrm{O}_{12}$ & $\mathrm{LiCl}-\mathrm{KCl}$ & $800-900$ & $1-5$ & Plates & 60 \\
$\mathrm{Ca}_{10}\left(\mathrm{PO}_{4}\right)_{6}(\mathrm{OH})_{2}$ & $\mathrm{~K}_{2} \mathrm{SO}_{4}$ & $1080-1200$ & $0.5-3$ & Whiskers & 61 \\
$\mathrm{CaSiO}_{3}$ & $\mathrm{NaCl}-\mathrm{KCl}$ & $850-1000$ & $1-48$ & Whiskers & 62 \\
$\mathrm{CaWO}_{4}$ & $\mathrm{KCl}$ & 900 & 5 & Whiskers & 63 \\
$\mathrm{Al}_{2} \mathrm{O}_{3}$ & $\mathrm{AlF}$ & $1000-1200$ & 3 & Plates & 64 \\
$\mathrm{SrFe}_{12} \mathrm{O}_{19}$ & $\mathrm{NaCl}-\mathrm{KCl}$ & $800-1100$ & $0.5-3$ & Plates & 65 \\
$\mathrm{BaFe}_{12} \mathrm{O}_{19}$ & $\mathrm{NaCl}-\mathrm{KCl}$ & $800-1100$ & $0.5-3$ & Plates & 65 \\
$\mathrm{Sr}_{3} \mathrm{Ti}_{2} \mathrm{O}_{7}$ & $\mathrm{NaCl}-\mathrm{KCl}$ & 1200 & 8 & Plates & 66 \\
$\mathrm{~K}_{2} \mathrm{Ti}_{4} \mathrm{O}_{9}$ & $\mathrm{~K}_{2} \mathrm{MoO}$ & 1150 & 4 & Whiskers & 67 \\
\hline
\end{tabular}

Table 2.3 : Summary of various molten salt reactions for the synthesis of powders with anisotropic particle shapes.

Crystal faces that have high lattice densities typically form the habit faces of a growing particle [68]. However, for a given material, the relative growth rates of the crystal faces can be altered by a variety of extrinsic factors. For example, impurities in the solution can often retard the growth of a crystal in certain directions [69] and the use of a different solvent or flux can sometimes result in a change of crystal habit $[61,68]$. In addition, the degree of supersaturation of a solution or melt can also exert a significant 
influence since the difference in the relative growth rates of the crystal faces decreases with higher levels of supersaturation [61].

An example of the effect of impurities on the growth habit of crystals is provided by Kawamura et al. [69] who found that the addition of trivalent cations to $\mathrm{SnO}_{2}$ in a $\mathrm{SnO}_{2}$ $\mathrm{Cu}_{2} \mathrm{O}$ flux system changed the crystal habit from prismatic to acicular needles. The work of Tas [61] on the synthesis of calcium hydroxyapatite whiskers $\left(\mathrm{Ca}_{10}\left(\mathrm{PO}_{4}\right)_{6}(\mathrm{OH})_{2}\right)$ by molten salt processing clearly demonstrated the important role played by the choice of flux. The use of $\mathrm{K}_{2} \mathrm{SO}_{4}$ resulted in the formation of short whiskers over the temperature range of 1080 to $1200^{\circ} \mathrm{C}$. However, the use of $\mathrm{KCl}, \mathrm{KBr}$, $\mathrm{CaCl}_{2}$, or $\mathrm{Na}_{2} \mathrm{SO}_{4}$ merely resulted in the formation of large single crystal particles.

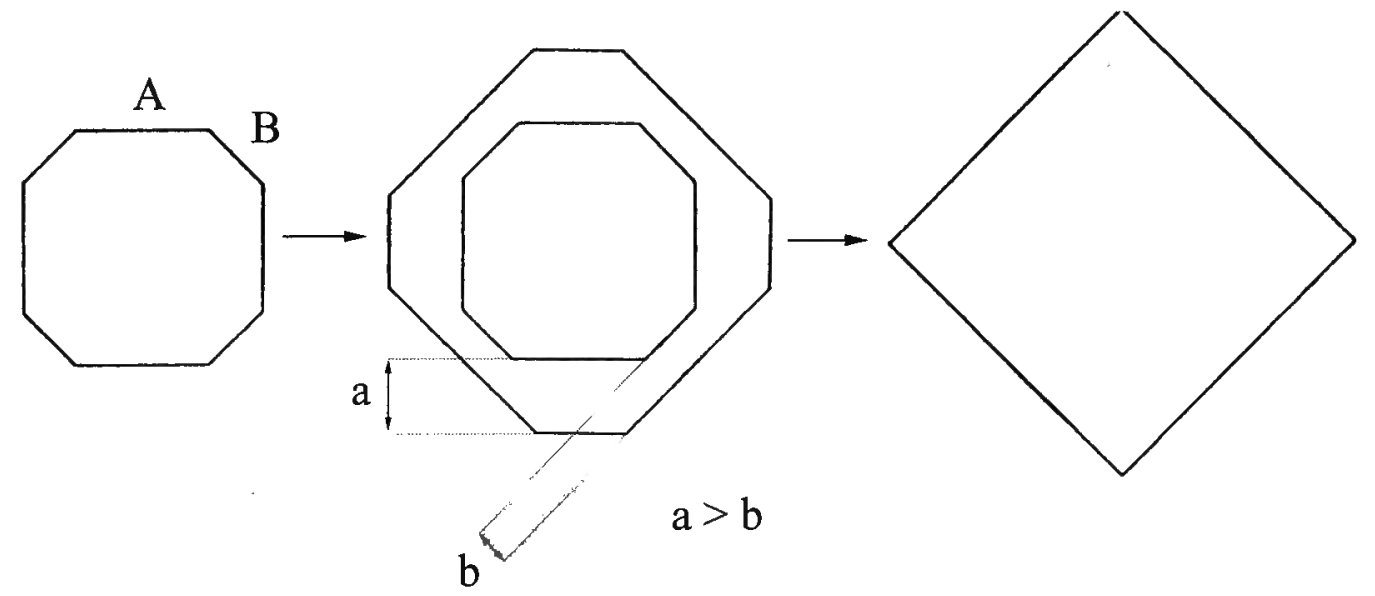

Figure 2.3 : Schematic illustration of a growing crystal demonstrating the disappearance of fast growing crystal faces. Adapted from [68]. 


\section{Chapter 3}

\section{Zirconia}

\section{(3.1) Introduction}

Materials based on zirconia $\left(\mathrm{ZrO}_{2}\right)$ are amongst the earliest studied class of oxide ceramics and have recently developed into an important class of advanced engineering materials. Zirconia was first identified by the German chemist Martin Heinrich Klaproth in 1789 as a reaction product obtained by heating gemstones with an alkali [70]. Since then, materials based on zirconia have found use in a wide variety of technological applications due to its unique combination of physical and chemical properties.

\section{(3.1.1) Engineering Applications}

Zirconia ceramics are primarily employed in traditional applications that take advantage of its intrinsic physical properties, such as its white colour, hardness, and refractory nature. These applications include use as paint pigments, abrasives, and foundry sands. However, recognition that the mechanical and electrical properties of zirconia can effectively be controlled through alloying and thermal treatments has led to the development of a wide range of advanced engineering applications [71].

Interest in zirconia as an advanced engineering material is primarily due to the phenomenon of transformation toughening, which allows the manufacture of ceramic materials with superior mechanical properties. In transformation toughened zirconia, metastable tetragonal precipitates within a cubic matrix transform to the monoclinic structure when the matrix constraint is relieved by the advancement of a crack through the material. The stress field associated with the transformation opposes further propagation of the crack, thus enhancing the toughness. Toughened zirconia ceramics find application as cutting tools, dies, and as automotive components [72].

Zirconia ceramics can be rendered electrically conductive at high temperatures through the addition of suitable dopants. The presence of dopant cations with a lower valence than the $\mathrm{Zr}^{4+}$ ion results in the formation of oxygen vacancies, which allows high levels 
of ionic mobility. Ionically conducting zirconia finds application as a solid state electrolyte in oxygen sensors, fuel cells, and furnace elements [73].

Zirconia has attracted considerable interest for applications as a catalyst and also as a support material for catalysts. This is largely a consequence of its high thermal stability and the amphoteric nature of its surface hydroxyl groups. Zirconia has been shown to catalyze a wide variety of reactions, including hydrogenation of olefins [74], isomerisation of olefins [75], and the dehydration of alcohols [76]. Reactions such as Fischer-Tropsch synthesis [77], methanol synthesis [78] and hydrodesulfurisation [79] have been reported to proceed with higher rates and selectivity with catalysts that use zirconia as a support.

\section{(3.1.2) Mineral Sources and Refining}

Zirconia is primarily derived from two main mineral sources, zircon $\left(\mathrm{ZrSiO}_{4}\right)$ and baddeleyite (impure monoclinic $\mathrm{ZrO}_{2}$ ). Of these two, zircon is of the greatest commercial significance. Chemical techniques for the decomposition of zircon use elevated processing temperatures and aggressive reagents. The two most commonly employed chemical processing methods are chlorination and alkali fusion. The chlorination process involves reaction of an intimate mixture of zircon and carbon in the presence of chlorine gas.

$$
\mathrm{ZrSiO}_{4}+4 \mathrm{C}+4 \mathrm{Cl}_{2(\mathrm{~g})} \rightarrow \mathrm{ZrCl}_{4}+\mathrm{SiCl}_{4}+4 \mathrm{CO}_{(\mathrm{g})}
$$

The $\mathrm{ZrCl}_{4}$ product phase is recovered by selective condensation and thereby separated from the major impurities. Subsequent hydrolysis results in the formation of $\mathrm{ZrOCl}_{2}$, which can then be used for the preparation of $\mathrm{ZrO}_{2}$ powders through chemical precipitation. Alkali fusion uses solid-state reaction with $\mathrm{NaOH}$ to decompose $\mathrm{ZrSiO}_{4}$ into $\mathrm{Na}_{2} \mathrm{ZrO}_{3}$ and $\mathrm{Na}_{2} \mathrm{SiO}_{3}$.

$$
\mathrm{ZrSiO}_{4}+4 \mathrm{NaOH} \rightarrow \mathrm{Na}_{2} \mathrm{ZrO}_{3}+\mathrm{Na}_{2} \mathrm{SiO}_{3}+2 \mathrm{H}_{2} \mathrm{O}_{(\mathrm{g})}
$$

Subsequent leaching with water removes the $\mathrm{Na}_{2} \mathrm{ZrO}_{3}$ by-product and hydrolyses the $\mathrm{Na}_{2} \mathrm{ZrO}_{3}$ to form hydrated $\mathrm{ZrO}_{2}$, which can then be calcined to yield an impure oxide. 
The manufacture of zirconia ceramics for advanced engineering applications typically imposes stringent constraints on the purity and morphology of the precursor powder. As a result, zirconia extracted from $\mathrm{ZrSiO}_{4}$ ore is invariably subjected to further chemical processing. This usually entails conversion to a soluble precursor, such as $\mathrm{ZrCl}_{4}$ or $\mathrm{ZrOCl}_{2}$, which can then used for the manufacture of high quality zirconia powders through chemical precipitation [8].

\section{(3.2) Crystallography}

Under atmospheric pressure, pure $\mathrm{ZrO}_{2}$ exists as one of three related crystal structures depending on the temperature. At low temperatures, the equilibrium structure is monoclinic (space group P21/c). On heating, the monoclinic structure transforms martensitically to tetragonal $(\mathrm{P} 42 / \mathrm{nmc})$ at approximately $1170^{\circ} \mathrm{C}$. At $2370^{\circ} \mathrm{C}$ the tetragonal structure transforms to cubic $(\mathrm{Fm} 3 \mathrm{~m})$, which remains stable until melting at $2680^{\circ} \mathrm{C}[71,80]$.

The cubic structure of $\mathrm{ZrO}_{2}$ consists of a face centered cubic array of $\mathrm{Zr}^{4+}$ ions with $\mathrm{O}^{2-}$ ions occupying the tetrahedral interstices. Each $\mathrm{Zr}^{4+}$ ion is in eightfold coordination with the $\mathrm{O}^{2-}$ ions. As illustrated in Figure 3.1, the monoclinic and tetragonal structures can simply be regarded as distortions of this cubic structure. In the monoclinic structure, each $\mathrm{Zr}^{4+}$ ion is in sevenfold coordination with the $\mathrm{O}^{2-}$ ions. The tetragonal structure is essentially an intermediate between the monoclinic and cubic forms with each $\mathrm{Zr}^{4+}$ ion being surrounded by eight $\mathrm{O}^{2-}$ ions.

At room temperature, pure $\mathrm{ZrO}_{2}$ undergoes two phase transformations with increasing pressure. At approximately $3.5 \mathrm{GPa}$, monoclinic $\mathrm{ZrO}_{2}$ undergoes a phase transformation to a structure that has been described by different authors as either tetragonal or centrosymmetric orthorhombic. Above $15 \mathrm{GPa}$, the equilibrium phase has the orthorhombic cotunnite structure [80]. 


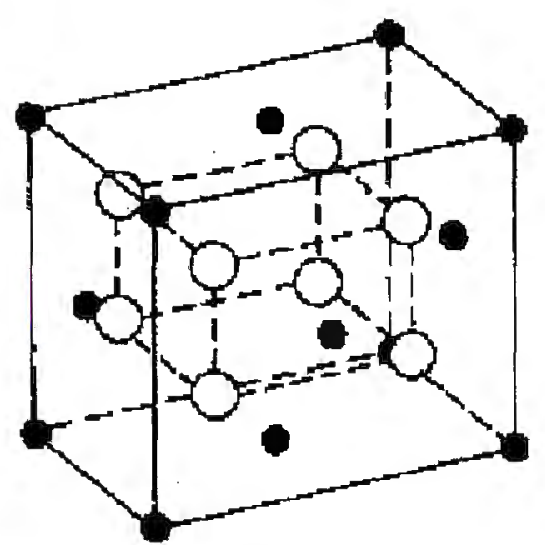

(a) Cubic

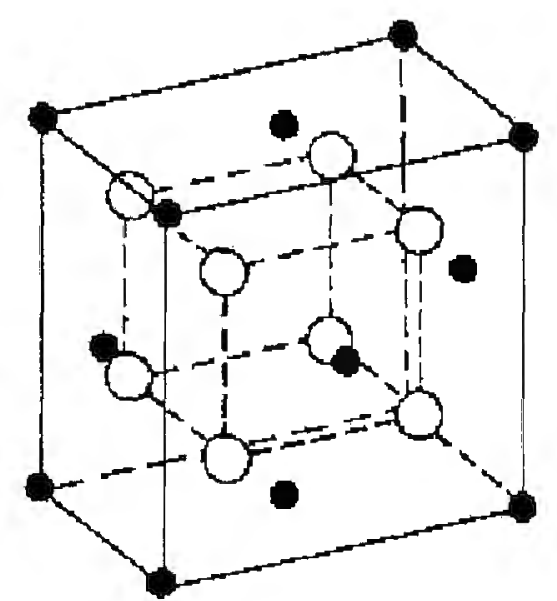

(b) Tetragonal

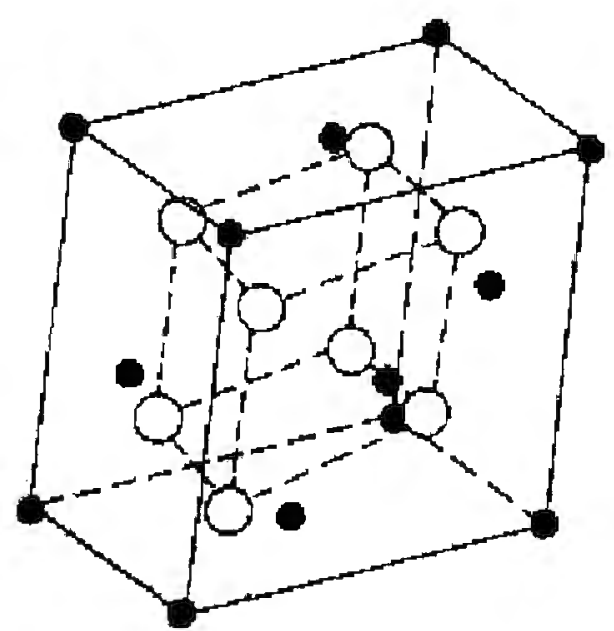

(c) Monoclinic

Figure 3.1 : Schematic illustration of the low pressure polymorphs of pure $\mathrm{ZrO}_{2}$. Closed circles are $\mathrm{Zr}^{4+}$ ions and open circles are $\mathrm{O}^{2-}$ ions [71]. 


\section{(3.3) Stabilised Zirconia Systems}

The chemical inertness and refractory nature of $\mathrm{ZrO}_{2}$ renders the material attractive for high temperature applications. However, the occurrence of the martensitic tetragonalmonoclinic phase transformation during thermal cycling inevitably results in cracking due to the associated volumetric strains. Practical application of $\mathrm{ZrO}_{2}$ therefore typically requires that the high temperature crystal polymorphs be stabilised through alloying with alkaline earth or rare earth oxides [71].

It has been suggested that the formation of oxygen vacancies resulting from doping with aliovalent oxides is the cause of cubic phase stabilisation. This is consistent with the observation that non-stoichiometry of oxygen ions stabilises the cubic phase to approximately $1525^{\circ} \mathrm{C}$, which is well below the transformation temperature of $2377^{\circ} \mathrm{C}$ for the stoichiometric compound. In the monoclinic form the zirconium ions are in sevenfold coordination whereas they are in eightfold coordination for the tetragonal and cubic structures. High concentrations of oxygen vacancies allow the zirconium atoms to have less than 8 neighbours within the framework of the higher symmetry structures [80].

Stabilised zirconia ceramics are commonly classified according to their phase content, which depends primarily on the dopant concentration. A sufficiently high concentration of stabiliser yields fully stabilised (FS) zirconia ceramics that consist solely of the cubic phase. Transformation toughened zirconia ceramics, which contain both cubic and tetragonal phases, are referred to as partially stabilised (PSZ). Single-phase ceramics based on tetragonal solid solutions are designated as tetragonal zirconia polycrystals (TZP) [72].

\section{(3.3.1) Partially Stabilised Zirconia}

The microstructure of PSZ consists of cubic grains with a fine dispersion of metastable tetragonal precipitates. PSZ ceramics are manufactured from suitable compositions by a three stage process consisting of high temperature sintering, quenching, and subsequent ageing. Sintering of the ceramic is performed at temperatures within the cubic phase field, thus forming a homogeneous cubic matrix. Rapid cooling through the cubic plus 
tetragonal phase field results in precipitation fine tetragonal particles. Subsequent ageing is then used to grow the tetragonal precipitates, thus developing the microstructure required for transformation toughening.

The most commonly used system for manufacturing PSZ ceramics is $\mathrm{MgO}-\mathrm{ZrO}_{2}$. As shown in Figure 3.2, the $\mathrm{MgO}-\mathrm{ZrO}_{2}$ phase diagram contains a large cubic phase field, which in conjunction with the slow nature of the eutectoid transformation enables the preparation of zirconia ceramics with a fully cubic structure. Common Mg-PSZ compositions contain $8-10 \mathrm{~mol} \% \mathrm{MgO}$ and are sintered within the cubic phase field at temperatures between 1700 and $1800^{\circ} \mathrm{C}$ [71].

\section{(3.3.2) Tetragonal Zirconia Polycrystal}

TZP ceramics are characterised by a microstructure that consists of fine tetragonal grains. These materials are usually sintered at temperatures that either lie within the tetragonal phase field or just within the tetragonal plus cubic phase field. Provided that a sufficiently small grain size is maintained, a single phase tetragonal structure is retained on cooling to room temperature.

As shown in Figure 3.3, approximately $2.5 \mathrm{~mol} \%$ of $\mathrm{Y}_{2} \mathrm{O}_{3}$ can be taken into solid solution. Typical Y-TZP ceramics therefore usually contain less than $3 \mathrm{~mol} \% \mathrm{Y}_{2} \mathrm{O}_{3}$. Higher concentrations of $\mathrm{Y}_{2} \mathrm{O}_{3}$ stabiliser result in formation of the cubic phase, which reduces the amount of tetragonal available for transformation toughening and therefore degrades the mechanical properties [71].

\section{(3.4) Particle Size Stabilisation}

In coarse grained $\mathrm{ZrO}_{2}$, the tetragonal phase is only stable at high temperatures and cannot be quenched to room temperature. However, low temperature synthesis of nanoparticulate $\mathrm{ZrO}_{2}$ is often found to result in formation of powders with the tetragonal structure rather than the expected monoclinic structure [81-84]. This phenomenon is of significant interest as the technological applications of $\mathrm{ZrO}_{2}$ depend strongly on an ability to control the phase transformation behaviour. 


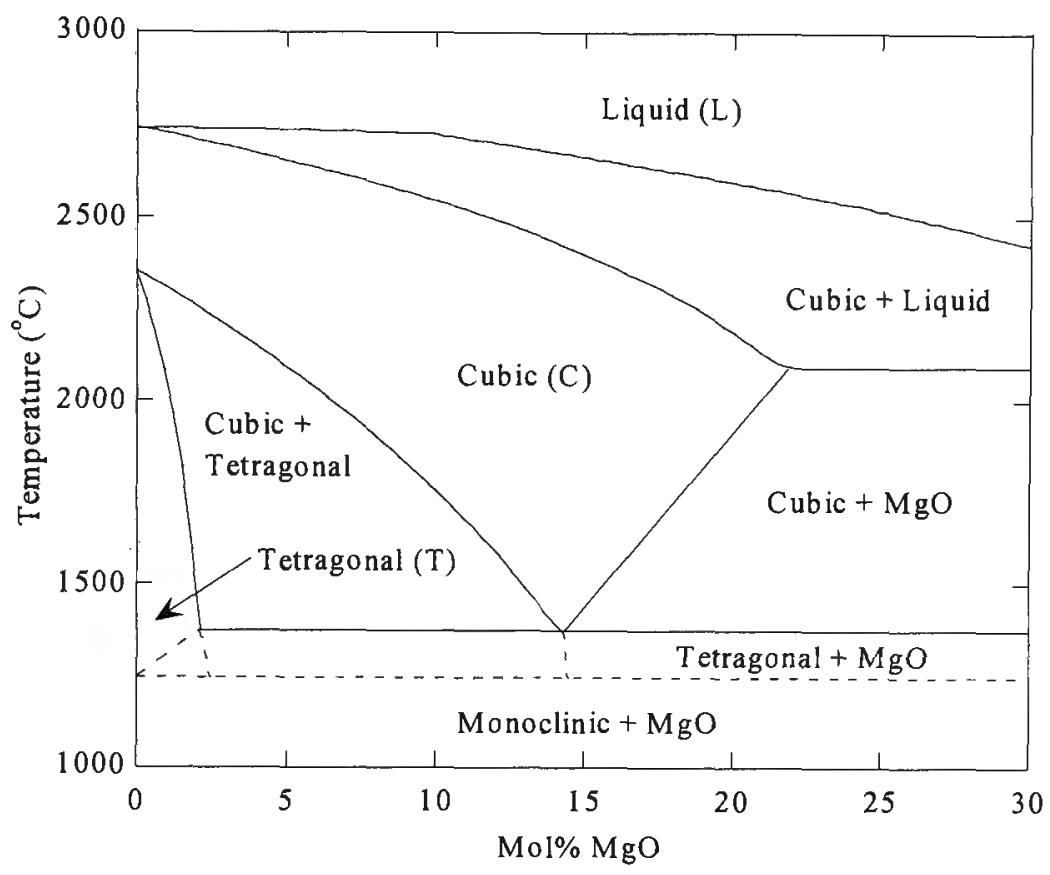

Figure 3.2 : Equilibrium phase diagram for the $\mathrm{MgO}-\mathrm{ZrO}_{2}$ system. Adapted from [71].

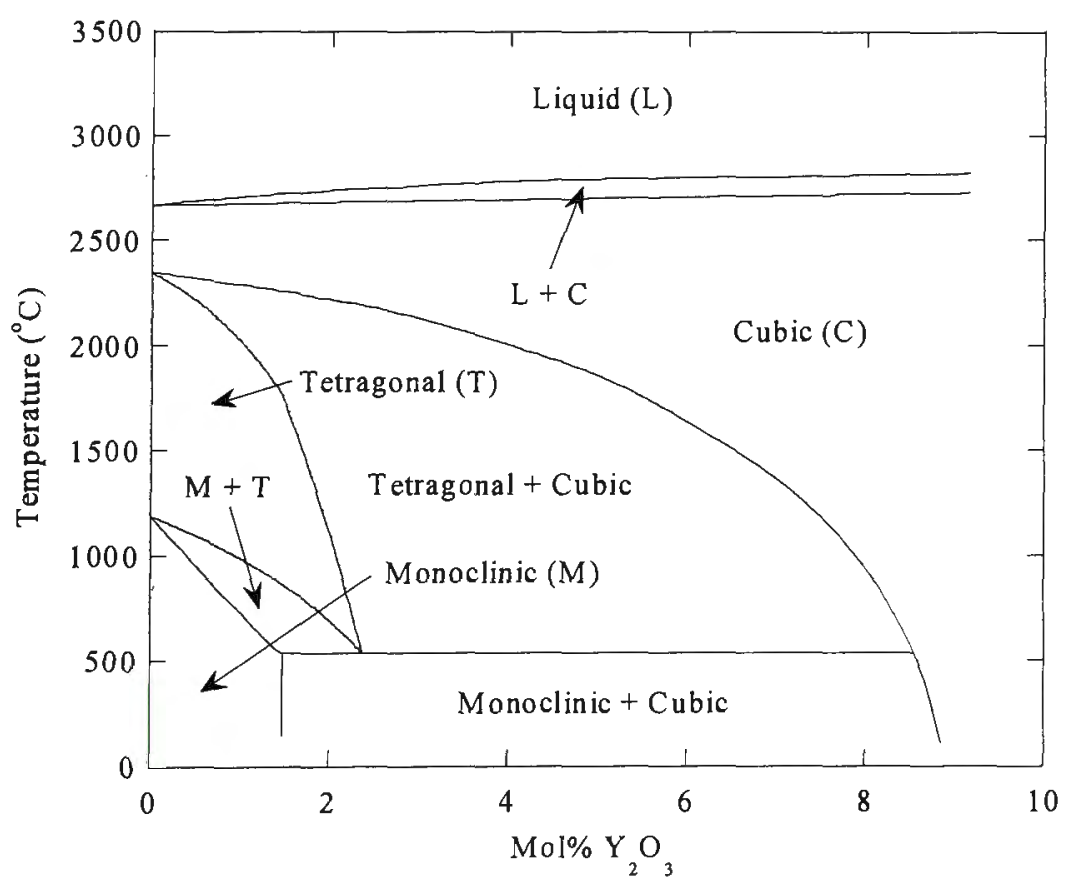

Figure 3.3 : Equilibrium phase diagram for the $\mathrm{Y}_{2} \mathrm{O}_{3}-\mathrm{ZrO}_{2}$ system. Adapted from [71]. 
Formation of the tetragonal phase has been attributed to either the non-equilibrium nature of the synthesis techniques or to an enhancement in the thermodynamic stability of the tetragonal phase in nanoparticulate $\mathrm{ZrO}_{2}$. The former explanation asserts that the tetragonal phase is metastable and that its formation is kinetically favoured over the equilibrium monoclinic structure for certain synthesis conditions [84]. The latter explanation holds that a reduction in the particle size of $\mathrm{ZrO}_{2}$ reduces the tetragonal to monoclinic transformation temperature, which renders the tetragonal phase stable at room temperature for a sufficiently small particle size [81-84].

\section{(3.4.1) Kinetic Factors}

Techniques for the synthesis of nanoparticulate $\mathrm{ZrO}_{2}$ typically employ low processing temperatures. As a result, it is unlikely that sufficient diffusion of oxygen can occur to fully saturate the structure. It has therefore been suggested that the tetragonal phase is actually an oxygen deficient form, which is stabilised by oxygen vacancies [81]. Supporting this view, Collins and Bowman [85] found that pyrolysis of a zirconium alkoxide under oxygen yielded the monoclinic phase whereas pyrolysis under a nitrogen atmosphere retained the tetragonal structure.

The most commonly used synthesis technique for manufacturing nanoparticulate $\mathrm{ZrO}_{2}$ is chemical precipitation. This technique typically results in the formation of an amorphous precursor which requires subsequent calcination to form the final $\mathrm{ZrO}_{2}$ product phase. A study by Livage et al. [86] found that the interatomic $\mathrm{Zr}-\mathrm{Zr}$ and $\mathrm{Zr}-\mathrm{O}$ distances in an amorphous $\mathrm{ZrO}_{2}$ gel were similar to those in the final tetragonal structure, which formed on subsequent calcination. This similarity in short-range order implies that the energy barrier between the amorphous and tetragonal structures is less than that for the monoclinic. As a result, it could therefore reasonably be expected that amorphous $\mathrm{ZrO}_{2}$ gels will tend to crystallise with the tetragonal structure.

\section{(3.4.2) Surface Energy Effects}

The stability of the tetragonal phase in nanoparticulate $\mathrm{ZrO}_{2}$ at temperatures below the equilibrium monoclinic-tetragonal transformation temperature has been attributed to the lower specific surface energy of the tetragonal phase compared to the monoclinic. As a 
consequence of this difference in surface energy there should exist a critical particle radius below which the tetragonal structure is stable for a given temperature [81-84]. This can be derived by considering the free energy of an unconstrained spherical particle, which is given by the sum of the volumetric and surface free energy terms as shown by equation (3.3):

$$
\mathrm{G}=\frac{4}{3} \pi \mathrm{r}^{3} \mathrm{G}_{\mathrm{v}}+4 \pi \mathrm{r}^{2} \gamma
$$

where $r$ is the particle radius, $G_{v}$ is the free energy per unit volume of an "infinite" crystal, and $\gamma$ is specific surface energy of the crystal. The difference in free energy between the tetragonal and monoclinic polymorphs can therefore be given by equation (3.4):

$$
\Delta \mathrm{G}=\frac{4}{3} \pi \mathrm{r}^{3} \Delta \mathrm{G}_{\mathrm{v}}+4 \pi \mathrm{r}^{2} \Delta \gamma
$$

where $\Delta G_{v}$ is the free energy difference for the phase transformation per unit volume of an "infinite" crystal. It can be seen from equation (3.2) that if the tetragonal phase has a lower surface energy compared to the monoclinic then there exists a critical particle radius for which $\Delta \mathrm{G}=0$. Substituting for volume free energy term gives the relation between the critical particle radius and the effective transformation temperature, as shown by equation (3.5):

$$
r_{c}=\frac{-3 \Delta \gamma}{\Delta H\left(1-T / T_{b}\right)}
$$

where $\Delta \mathrm{H}$ is the heat of transformation per unit volume of an infinite crystal and $T_{b}$ is the transformation temperature of an infinite crystallite. Supporting evidence for the relation given by equation (3.5) has been provided by Garvie and Goss [83]. In their study, they found quantitative agreement between theoretical calculations and experimentally derived measurements. 
The surface energy differential between the monoclinic and tetragonal polymorphs has been used by several authors as an explanation for the stability of the tetragonal phase in nanoparticulate $\mathrm{ZrO}_{2}$ and its transformation behaviour. For example, Xie et al. [87] found that exposure of tetragonal $\mathrm{ZrO}_{2}$ to water resulted in extensive transformation to the monoclinic form without significant loss of specific surface area. This behaviour was explained as being due to a decrease in the surface energy differential as a result of water adsorption. As supporting evidence, it was noted that the heat adsorption for $\mathrm{CO}_{2}$ on monoclinic $\mathrm{ZrO}_{2}$ is higher than on the tetragonal phase [88].

Chraska et al. [89] investigated the structure of $\mathrm{ZrO}_{2}$ nanoparticles that were prepared by plasma-spraying of an organometallic precursor. Examination of the particles by transmission electron microscopy revealed that the smaller particles had the tetragonal structure whilst the larger particles were monoclinic. On annealing, the particles coarsened and the tetragonal particles underwent transformation to the monoclinic structure when the particle size approached $18 \mathrm{~nm}$. These results were interpreted as being consistent with the concept of surface energy stabilisation. 


\section{Chapter 4}

\section{Experimental Techniques}

\section{(4.1) Powder Synthesis}

\section{(4.1.1) Reagent Storage and Handling}

All of the chemical reagents that were used in the present study are characterised by airsensitivity. Consequently, all reagents were stored and handled within a high-purity argon filled glovebox. The atmosphere of the glovebox typically contained no more than $10 \mathrm{ppm}$ of either oxygen or water vapour.

\section{(4.1.2) Mechanochemical Processing}

Reactant mixtures were milled using a vibratory Spex 8000 mixer/mill. All millings used a $10 \mathrm{~g}$ powder charge with twenty $9.5 \mathrm{~mm}$ stainless steel balls as the grinding media. To ensure an inert atmosphere during mechanochemical processing, the grinding media and reactants were loaded into the milling vial whilst within the glovebox. Milling times ranged from 1 minute up to 24 hours.

The milling vial used for mechanochemical processing of the reactant mixtures was manufactured from hardened steel and was characterised by internal dimensions of $40 \mathrm{~mm}$ diameter and $60 \mathrm{~mm}$ length. The vial was sealed with a Viton (fluorosilicone) Oring and a locking collar.

In order to detect any combustion events during milling, the temperature of the vial was continuously monitored using a flexible K-type thermocouple attached to the outer surface. The temperature readings were logged as a function of time using a chart recorder. 


\section{(4.1.3) Heat-Treatment}

Milled reactant mixtures that used either $\mathrm{MgO}$ or $\mathrm{Li}_{2} \mathrm{O}$ as the exchange reagent were heat-treated using a tube furnace with a digital temperature controller. To prevent undesired interactions with the atmosphere, the powders were contained within a sealed heat-treatment tube that was loaded within the argon filled glovebox. In an effort to ensure uniform conditions between experiments, the controlling thermocouple was consistently located in the same place on the exterior of the tube for each heat treatment.

Reactant mixtures that used $\mathrm{LiOH}$ as the exchange reagent were either heat-treated under a flowing argon atmosphere or simply in air depending on whether the precursors had undergone complete reaction during milling. A sealed argon atmosphere could not be used in either case due to the evolution of water vapour. However, in cases where complete reaction occurred during milling an inert atmosphere was not required, as the $\mathrm{LiCl}$ reaction by-product does not react with the oxygen in air.

\section{(4.1.4) Washing and Drying}

In order to remove the salt by-product the reacted powders were repeatedly washed with deionised water in an ultrasonic bath. Following each wash, the powder was recovered from the solvent by centrifugation and decantation. Prior to drying the washed powders were rinsed with methanol in an effort to inhibit the subsequent formation of hard agglomerates. Rinsed powders were dried for several hours at approximately $80^{\circ} \mathrm{C}$ in air.

\section{(4.2) Characterisation Techniques}

\section{(4.2.1) X-Ray Diffraction}

X-ray diffraction (XRD) measurements were performed using a Siemens D5000 diffractometer with monochromatic $\mathrm{Cu}-\mathrm{K}_{\alpha}$ radiation. The XRD data was collected by computer using the Siemens "Diffrac" software package. The "EVA" program (version 2.2.0.0) was used for phase identification and also to strip the $\mathrm{Cu}-\mathrm{K}_{\alpha 2}$ diffraction lines in preparation for subsequent quantitative analysis of the peak broadening and integral 
peak intensities. Peak widths and integral peak intensities were measured by fitting Gaussian peak profiles to the measured patterns through use of the "WinFit" computer program [90].

Unwashed powder samples were expected to show air sensitivity due to the hygroscopic nature of the chemicals involved and were therefore analysed under a sealed argon atmosphere using an airtight sample holder. Washed powders were analysed in conventional open-air sample holders. For all measurements, a glass slide was used to flatten the powder into the sample holder, thus ensuring correct alignment of the powder surface inside the diffractometer.

Quantitative phase analysis of the washed zirconia powders was performed by measuring the integral intensities of the (111) and (111) monoclinic and (101) tetragonal diffraction peaks [91]. This allowed calculation of the monoclinic volume fraction, $\mathrm{v}_{\mathrm{m}}$, through equation (4.1):

$$
\mathrm{v}_{\mathrm{m}}=\frac{\mathrm{PX}_{\mathrm{m}}}{1+(P-1) X_{\mathrm{m}}}
$$

where $X_{\mathrm{m}}$ is the integrated intensity ratio, which is given by equation (4.2):

$$
X_{m}=\frac{I_{m}(\overline{1} 11)+I_{m}(111)}{I_{m}(\overline{1} 11)+I_{m}(111)+I_{t}(101)}
$$

The constant $\mathrm{P}$ in equation (4.1) essentially describes the deviation from linearity between the actual volume fraction of the monoclinic phase and the diffracted $\mathrm{x}$-ray intensity. This depends on the unit cell parameter and is therefore affected by composition [93]. In the present study, three different compositions were analysed, specially pure $\mathrm{ZrO}_{2}, \mathrm{Mg}-\mathrm{PSZ}$ (10mol.\%), and Y-TZP (3mol.\%). For each case, appropriate values for the constant were taken from those published by Toraya [92].

Crystallite sizes were determined from the peak broadening using the Scherrer equation [93]. 


$$
\mathrm{D}=\frac{0.9 \lambda}{\beta \cos \theta}
$$

where $D$ is the crystallite size, $\lambda$ is the wavelength, $\beta$ is the corrected peak width, and $\theta$ is the diffraction angle. The corrected peak breadth was determined from the measured peak breadth $\left(\mathrm{B}_{\mathrm{m}}\right)$ by subtracting out the instrumental broadening $\left(\mathrm{B}_{\mathrm{i}}\right)$ using equation (4.5):

$$
\beta=\sqrt{\mathrm{B}_{\mathrm{m}}^{2}-\mathrm{B}_{\mathrm{i}}^{2}}
$$

The value for the instrumental broadening was determined by measuring the peak profiles for a coarse grained $\mathrm{ZrO}_{2}$ sample that had been annealed at $1500^{\circ} \mathrm{C}$ for 2 hours.

\section{(4.2.2) Thermal Analysis}

Simultaneous thermogravimetric-differential thermal analysis (TG-DTA) of the asmilled powders was performed using a Rigaku Thermoflex system. In cases where airsensitivity of the sample was expected, the DTA crucible was loaded within the argon filled glovebox and the measurement performed under a flowing argon atmosphere. Non-sensitive samples were loaded and analysed in air. The majority of measurements were taken using a heating ramp rate of $20^{\circ} \mathrm{C}$ /minute. For all samples, the acquired data was normalised to a constant mass.

\section{(4.2.3) Electron Microscopy}

The particle size and morphology of the washed powders was examined by transmission electron microscopy (TEM) using a Philips 430 microscope with a beam energy of 300 $\mathrm{keV}$. Samples for TEM were prepared by ultrasonically dispersing the washed and dried powder in ethanol and then evaporating a drop of the dispersion on a carbon coated specimen grid. 


\section{(4.2.4) Specific Surface Area Measurement}

The specific surface area of the washed powders was measured by five-point BET gas adsorption using a Micromeritics Gemini 2360 instrument. Prior to analysis, the washed powders were vacuum degassed at $200^{\circ} \mathrm{C}$ for a minimum of 1 hour. The specific surface area was used to derive estimates of the average particle size by assuming that the powders consisted of separate spherical particles. The average particle size could therefore be related to the specific surface area by equation 4.5 :

$$
S=\frac{6}{\rho D}
$$

where $\mathrm{S}$ is the specific surface area, $\rho$ is the particle density, and $\mathrm{D}$ is the average particle diameter.

\section{(4.2.5) Chemical Analysis}

Washed and dried powder samples were analysed for contamination with iron from the grinding media and residual by-product salt using an ARL 3520 inductively coupled plasma atomic emission spectrometer (ICP-AES). All chemical analysis measurements were performed by the Chemistry Centre of Western Australia. 


\section{Chapter 5}

\section{Experimental Results}

\section{(5.1) Introduction}

\section{(5.1.1) Overview of Reaction Systems}

Table 5.1 shows a summary of the three basic mechanochemical reaction systems that were investigated in this study for the synthesis of $\mathrm{ZrO}_{2}$ powders. As shown, all three systems involve reaction of anhydrous $\mathrm{ZrCl}_{4}$ with an oxide exchange reagent and are characterised by a negative Gibbs free energy change, which indicates a strong driving force for chemical reaction. These three reaction systems were investigated with regard to the effect of milling time, dilution, and post-milling heat-treatment.

\begin{tabular}{lcc}
\hline Reaction Equation & $\Delta \mathrm{G}(\mathrm{kJ} /$ mole $)$ & $\Delta \mathrm{H}(\mathrm{kJ} /$ mole $)$ \\
\hline $\mathrm{ZrCl}_{4}+2 \mathrm{MgO} \rightarrow \mathrm{ZrO}_{2}+2 \mathrm{MgCl}_{2}$ & -196 & -198 \\
$\mathrm{ZrCl}_{4}+2 \mathrm{Li}_{2} \mathrm{O} \rightarrow \mathrm{ZrO}_{2}+4 \mathrm{LiCl}$ & -564 & -551 \\
$\mathrm{ZrCl}_{4}+4 \mathrm{LiOH} \rightarrow \mathrm{ZrO}_{2}+4 \mathrm{LiCl}+2 \mathrm{H}_{2} \mathrm{O}_{(\mathrm{g})}$ & -411 & -295 \\
\hline
\end{tabular}

Table 5.1 : Summary of mechanochemical reaction systems that were investigated for the synthesis of $\mathrm{ZrO}_{2}$ powders. $\Delta \mathrm{G}$ and $\Delta \mathrm{H}$ values were calculated for reaction at $100^{\circ} \mathrm{C}$.

Reaction systems for the synthesis of $\mathrm{MgO}$ and $\mathrm{Y}_{2} \mathrm{O}_{3}$ were also investigated. As shown in Table 5.2, these systems were essentially similar to those used for the manufacture of $\mathrm{ZrO}_{2}$ in that they involve reaction of anhydrous chloride precursors with an oxide exchange reagent and are thermodynamically favourable. These four systems were studied with the objective of combining them with $\mathrm{ZrO}_{2}$ synthesis reactions for the preparation of stabilised zirconia powders, specifically Mg-PSZ and Y-TZP.

No attempt was made to synthesise $\mathrm{Y}_{2} \mathrm{O}_{3}$ or $\mathrm{Y}$-TZP with $\mathrm{MgO}$ as the oxide exchange reagent since the reaction $2 \mathrm{YOCl}+\mathrm{MgO} \rightarrow \mathrm{Y}_{2} \mathrm{O}_{3}+\mathrm{MgCl}_{2}$ is characterised by a positive free energy change. 


\section{(5.1.2) Powder Contamination}

As was noted previously in Chapter 4, the reactant mixtures were milled using stainless steel grinding media, which inevitably led to contamination of the milled powders with iron. As would be expected, the degree of contamination was found to increase with the duration of milling. In addition, the final washed powders also contained small quantities of residual salt that were not completely removed by the washing process. Concentrations of residual salt were typically less than approximately $0.05 \mathrm{wt} . \%$.

\begin{tabular}{lcc}
\hline Reaction Equation & $\Delta \mathrm{G}(\mathrm{kJ} / \mathrm{mole})$ & $\Delta \mathrm{H}(\mathrm{kJ} / \mathrm{mole})$ \\
\hline $\mathrm{MgCl}_{2}+\mathrm{Li}_{2} \mathrm{O} \rightarrow \mathrm{MgO}+2 \mathrm{LiCl}$ & -184 & -179 \\
$2 \mathrm{YCl}_{3}+3 \mathrm{Li}_{2} \mathrm{O} \rightarrow \mathrm{Y}_{2} \mathrm{O}_{3}+6 \mathrm{LiCl}$ & -584 & -556 \\
$\mathrm{MgCl}_{2}+2 \mathrm{LiOH} \rightarrow \mathrm{MgO}+2 \mathrm{LiCl}+\mathrm{H}_{2} \mathrm{O}_{(\mathrm{g})}$ & -107 & -49 \\
$2 \mathrm{YCl}_{3}+6 \mathrm{LiOH} \rightarrow \mathrm{Y}_{2} \mathrm{O}_{3}+6 \mathrm{LiCl}+3 \mathrm{H}_{2} \mathrm{O}_{(\mathrm{g})}$ & -359 & -171 \\
\hline
\end{tabular}

Table 5.2 : Summary of mechanochemical reaction systems that were investigated for the synthesis of $\mathrm{MgO}$ and $\mathrm{Y}_{2} \mathrm{O}_{3}$ powders. $\Delta \mathrm{G}$ and $\Delta \mathrm{H}$ values were calculated for reaction at $100^{\circ} \mathrm{C}$.

The degree of iron contamination was found to be most severe for undiluted LiOH reactant mixtures that were subjected to continued milling after the combustion event. Contamination increased near linearly from negligible levels immediately following combustion to almost $1 \mathrm{wt} . \%$ of iron by 24 hours of milling. Levels of iron contamination were significantly less for diluted $\mathrm{LiOH}$ systems and those based on $\mathrm{Li}_{2} \mathrm{O}$ and $\mathrm{MgO}$, which typically did not exceed $0.1 \mathrm{wt} . \%$.

\section{(5.2) Magnesium Oxide Reactions}

\section{(5.2.1) Reaction Behaviour}

Measurement of the vial temperature during milling of $\mathrm{ZrCl}_{4}+2 \mathrm{MgO}$ revealed only a gradual increase up to a steady-state value of approximately $39^{\circ} \mathrm{C}$ as a result of the heat generated by collisions of the grinding media. No sudden temperature spike 
characteristic of combustive reaction was observed, which indicates that chemical reaction of the precursors occurred in a gradual manner. XRD analysis showed that the reaction was essentially complete following 6 hours of milling and yielded a nanocrystalline powder consisting of $\mathrm{MgCl}_{2}$ and ultrafine particles of tetragonal $\mathrm{ZrO}_{2}$. The as-milled $\mathrm{ZrO}_{2}$ product phase was highly reactive and as a result underwent amorphisation during subsequent washing with deionised water. Calcination of the washed powder resulted in the crystallisation of tetragonal $\mathrm{ZrO}_{2}$.

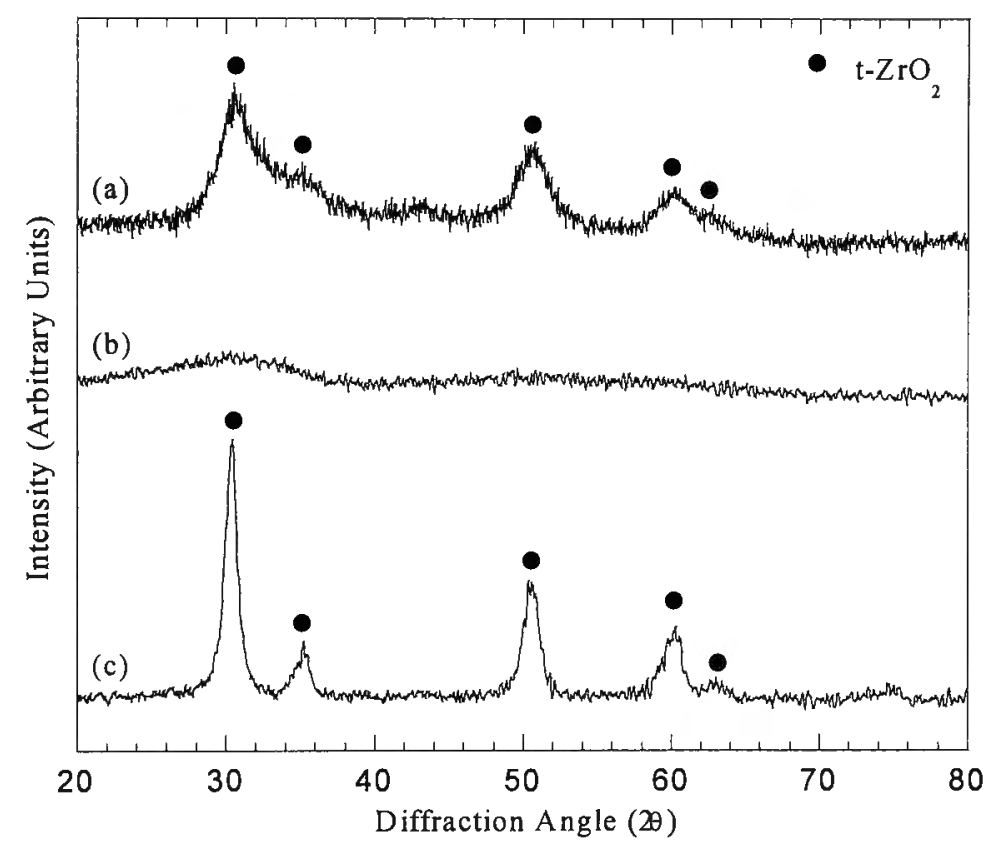

Figure 5.1 : XRD pattern of $\mathrm{ZrCl}_{4}+2 \mathrm{MgO}$ following (a) milling for 6 hours, (b) washing, and (c) calcination at $500^{\circ} \mathrm{C}$ for 1 hour

Illustrating this behaviour, Figure 5.1 shows the $\mathrm{XRD}$ pattern of $\mathrm{ZrCl}_{4}+2 \mathrm{MgO}$ following (a) milling for 6 hours, (b) washing, and (c) calcination at $500^{\circ} \mathrm{C}$ for 1 hour. The pattern of the as-milled powder consists of broad diffraction peaks of low intensity corresponding to tetragonal $\mathrm{ZrO}_{2}$. No peaks corresponding to the $\mathrm{MgCl}_{2}$ by-product phase are evident. Following washing no diffraction peaks of any crystalline phase remained, indicating the occurrence of amorphisation of the tetragonal $\mathrm{ZrO}_{2}$ product and the successful removal of the salt by-product. Calcination of the washed powder resulted in the reappearance of diffraction peaks corresponding to tetragonal $\mathrm{ZrO}_{2}$. Quantitative analysis of the peak broadening with the Scherrer equation gave an average crystallite size of approximately $10.8 \mathrm{~nm}$. 
Although the mechanically induced disorder of the as-milled powder prevented accurate quantitative analysis of the peak broadening, it nevertheless appears that the crystallite size of the as-milled $\mathrm{ZrO}_{2}$ reaction product was significantly smaller than that of the final calcined powder. This indicates that the amorphisation reaction during washing was accompanied by coarsening of the $\mathrm{ZrO}_{2}$ particles.

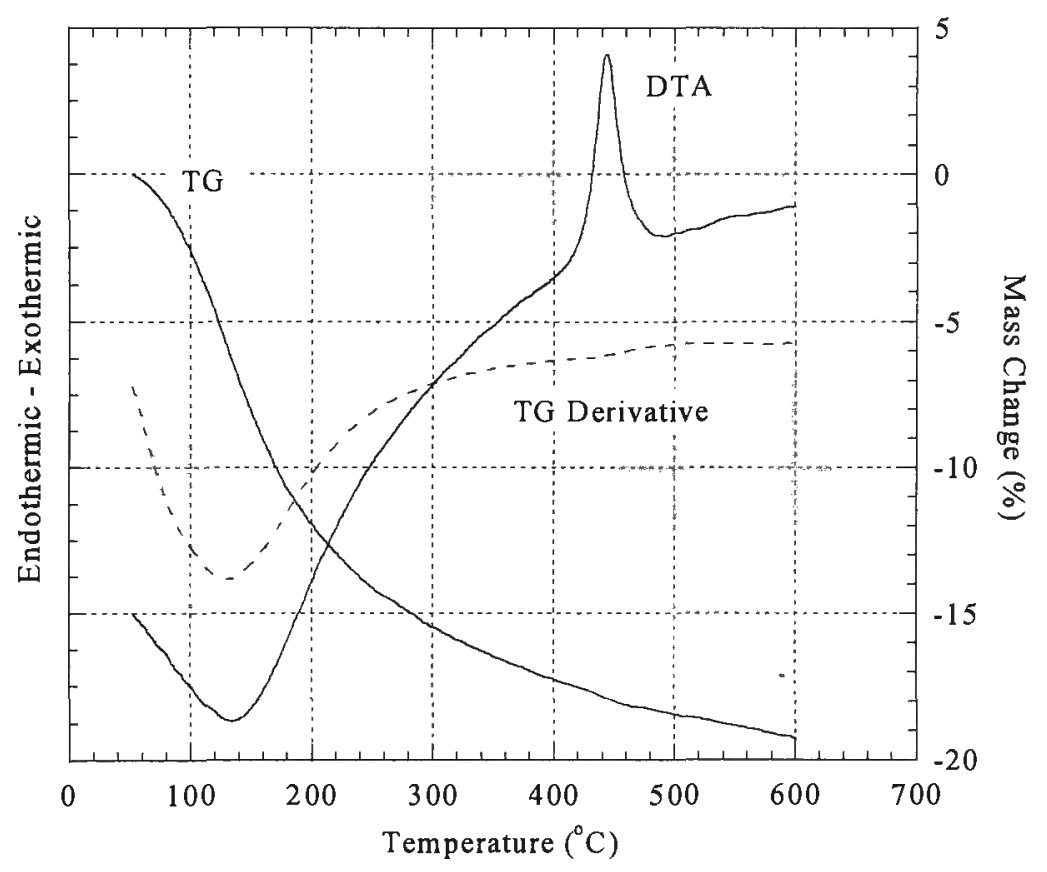

Figure 5.2 : Simultaneous TG-DTA traces of milled and washed $\mathrm{ZrCl}_{4}+2 \mathrm{MgO}$.

No diffraction peaks corresponding to the $\mathrm{MgCl}_{2}$ by-product phase were apparent in the XRD pattern of the as-milled powder. This is primarily attributable to peak overlap since all of the major $\mathrm{MgCl}_{2}$ diffraction peaks are in close proximity to those of tetragonal $\mathrm{ZrO}_{2}$. It is also likely that $\mathrm{MgCl}_{2}$ phase was highly disordered as a result of the milling action, thus reducing the intensity of any diffraction peaks. However, despite these factors, the formation of $\mathrm{MgCl}_{2}$ during milling can nevertheless be inferred from ICP-AES analysis of the wash water solution, which showed the ratio of $\mathrm{Mg}$ to $\mathrm{Cl}$ ion content to be consistent with the expected aqueous $\mathrm{MgCl}_{2}$ solution.

The occurrence of an amorphisation reaction during washing was confirmed by thermal analysis of the as-milled and washed powders. The DTA trace of the as-milled powder revealed only an endothermic event corresponding to melting of the $\mathrm{MgCl}_{2}$ by-product phase. However, simultaneous TG-DTA of the washed powder revealed thermal 
behaviour similar to that exhibited by amorphous $\mathrm{Zr}(\mathrm{OH})_{4}$ gels prepared by aqueous chemical precipitation. As shown in Figure 5.2, the DTA trace consists of a broad endothermic peak at low temperature followed by an exothermic peak at approximately $420^{\circ} \mathrm{C}$, which corresponds to crystallisation of the tetragonal $\mathrm{ZrO}_{2}$ phase from the amorphous precursor.

The endothermic peak in the DTA trace appears to correlate with a maximum in the rate of mass loss and can therefore most likely be attributed to the loss of physisorbed and chemically bound water molecules that were acquired during the washing procedure. The TG trace revealed a continuous mass loss over the entire heating ramp that totalled approximately $19.2 \%$ by $600^{\circ} \mathrm{C}$, which corresponds to approximately 1.63 moles of $\mathrm{H}_{2} \mathrm{O}$ per mole of $\mathrm{ZrO}_{2}$.

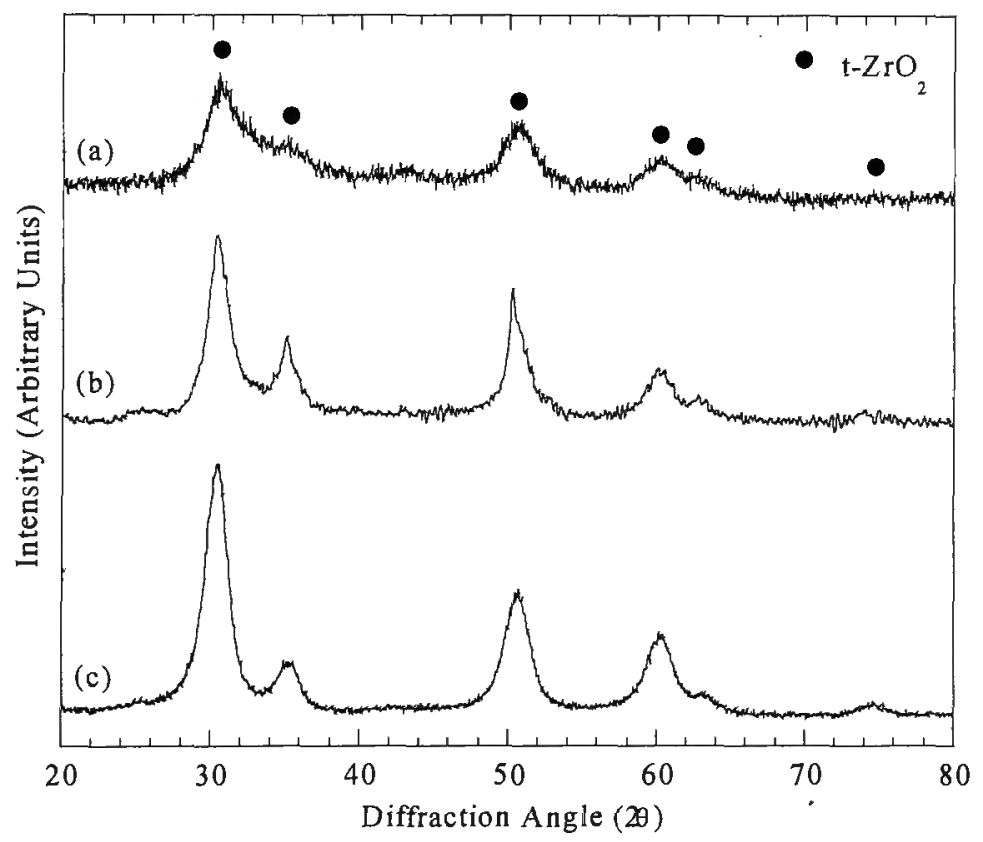

Figure 5.3 : XRD pattern of $\mathrm{ZrCl}_{4}+2 \mathrm{MgO}$ following (a) milling for 6 hours, (b) heattreatment at $500^{\circ} \mathrm{C}$ for 1 hour under a sealed argon atmosphere, and (c) washing.

Low temperature heat treatment of the as-milled powder was found to substantially decrease the reactivity of the ultrafine $\mathrm{ZrO}_{2}$ particles towards the wash water. This is illustrated in Figure 5.3, which shows the XRD pattern of $\mathrm{ZrCl}_{4}+2 \mathrm{MgO}$ following (a) milling for 6 hours, (b) heat-treatment at $500^{\circ} \mathrm{C}$ for 1 hour under a sealed argon atmosphere, and (c) washing. Heat-treatment of the as-milled powder resulted in 
significant improvement of the peak definition, which indicates that recovery of mechanically induced disorder occurred. Subsequent washing of this heat-treated powder yielded an ultrafine powder of crystalline tetragonal $\mathrm{ZrO}_{2}$ without need of subsequent calcination. Quantitative analysis of the peak broadening with the Scherrer equation gave an average crystallite size of approximately $5.4 \mathrm{~nm}$.

In contrast to the behaviour observed in Figure 5.1, washing of the heat-treated powder did not result in any significant alteration to the width of the $\mathrm{ZrO}_{2}$ diffraction peaks. As a result, it can reasonably be concluded that the crystallite size derived by quantitative analysis of the washed powder is representative of that formed mechanochemically during milling.

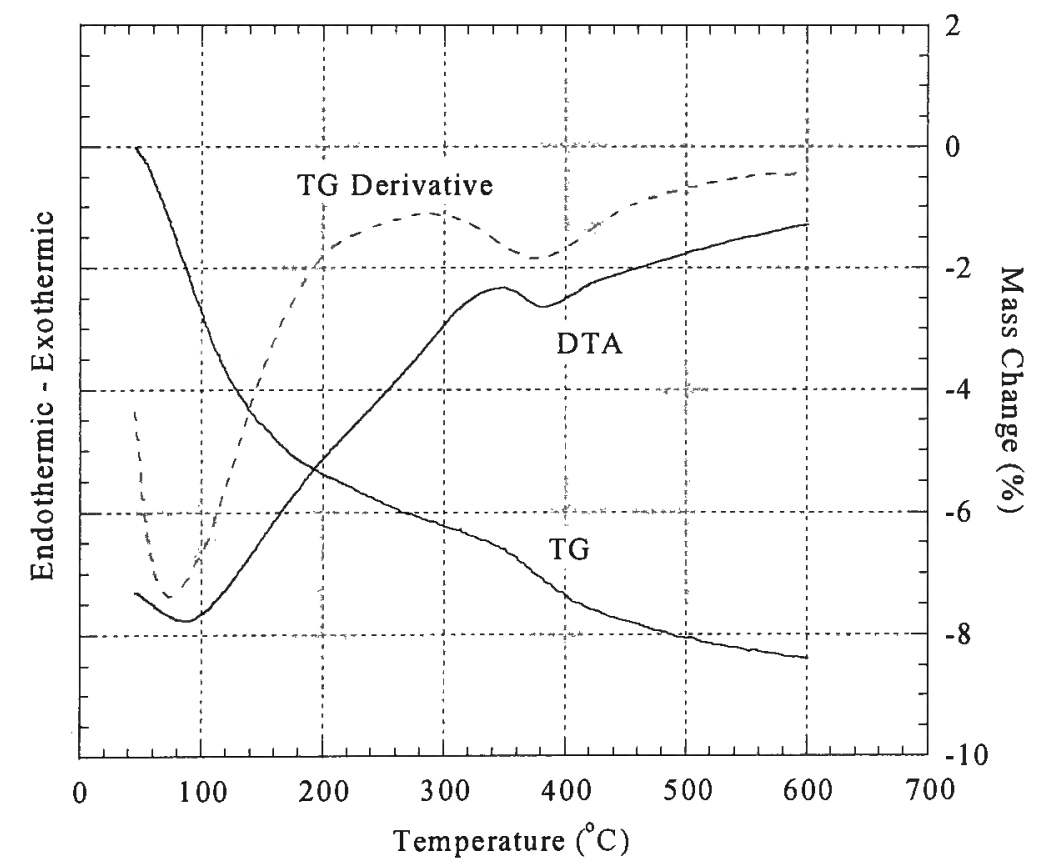

Figure 5.4 : Simultaneous TG-DTA traces of milled, heat-treated and washed $\mathrm{ZrCl}_{4}+$ $2 \mathrm{MgO}$.

As shown by TG-DTA in Figure 5.4, heat-treatment of the as-milled powder resulted in a significant change to the thermal behaviour of the final washed powder. Consistent with the crystallinity detected by XRD of the washed powder, no exothermic peak corresponding to crystallisation is evident. The DTA trace contains only two endothermic peaks, both of which appear to be associated with local maxima in the rate of mass loss. These two endotherms are attributable to the loss of physisorbed and 
chemisorbed water, respectively. The TG trace revealed a continuous mass loss over the entire heating ramp that reached approximately $8.8 \%$ by $600^{\circ} \mathrm{C}$, which corresponds to approximately 0.66 moles of $\mathrm{H}_{2} \mathrm{O}$ per mole of $\mathrm{ZrO}_{2}$.

\section{(5.2.2) Agglomeration Behaviour}

Post-milling heat treatment was found to have substantial impact on the formation of agglomerates in the final powder. The dried gel formed by water washing of the asmilled powder was in the form of hard irregular granules that required intensive grinding to be: reduced to a fine-grained powder whereas powders that were prepared with a post-milling heat-treatment were found to be soft and readily friable.

As shown by TEM in Figure 5.5 (a), powder prepared by washing of the as-milled powder is comprised of irregularly shaped agglomerates of high density with no readily apparent individual crystallites. In contrast, powder prepared with a post-milling heat treatment of $500^{\circ} \mathrm{C}$ for 1 hour, shown in Figure 5.5 (b), consists predominantly of distinct particles.

\begin{tabular}{llll}
\hline Post-Milling Treatment & $\begin{array}{l}\mathrm{S} \\
\left(\mathrm{m}^{2} / \mathrm{g}\right)\end{array}$ & $\begin{array}{l}\mathrm{D}_{\mathrm{BET}} \\
(\mathrm{nm})\end{array}$ & $\begin{array}{l}\mathrm{D}_{\mathrm{XRD}} \\
(\mathrm{nm})\end{array}$ \\
\hline Washed and calcined & 154.0 & 6.49 & 10.8 \\
Heat-treated and washed & 184.1 & 5.43 & 5.4 \\
Washed, methanol rinsed, and calcined & 173.9 & 5.75 & 10.0 \\
Heat-treated, washed, and methanol rinsed & 189.4 & 5.27 & 5.3 \\
\hline
\end{tabular}

Table 5.3 : XRD and BET crystallite sizes of dried $\mathrm{ZrO}_{2}$ powders prepared from asmilled $\mathrm{ZrCl}_{4}+2 \mathrm{MgO}$ using various post-milling treatments.

Rinsing of the washed powders with methanol prior to drying and calcination also had a significant effect on the agglomeration behaviour of the final powder. Methanol rinsed powders were found to be soft and were readily broken down with gentle grinding.

Figure 5.6 (c) shows a powder prepared from the as-milled reactant mixture with a methanol-rinsing step. The powder consists primarily of large rounded clusters within 
which individual particles can be discerned. Smaller clusters and individual particles can also be seen. Powder prepared with post-milling heat treatment and methanol rinsing, shown in Figure 5.5 (d), is of similar appearance to that shown in Figure 5.5 (b).

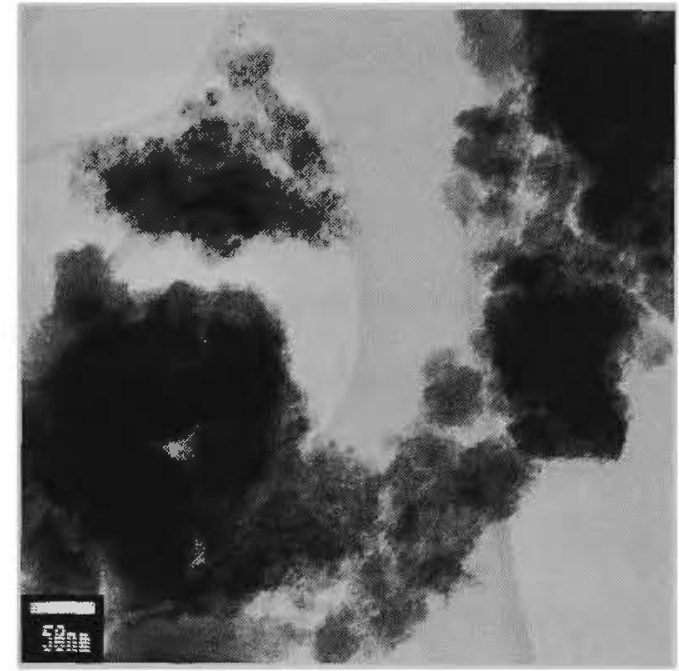

(a)

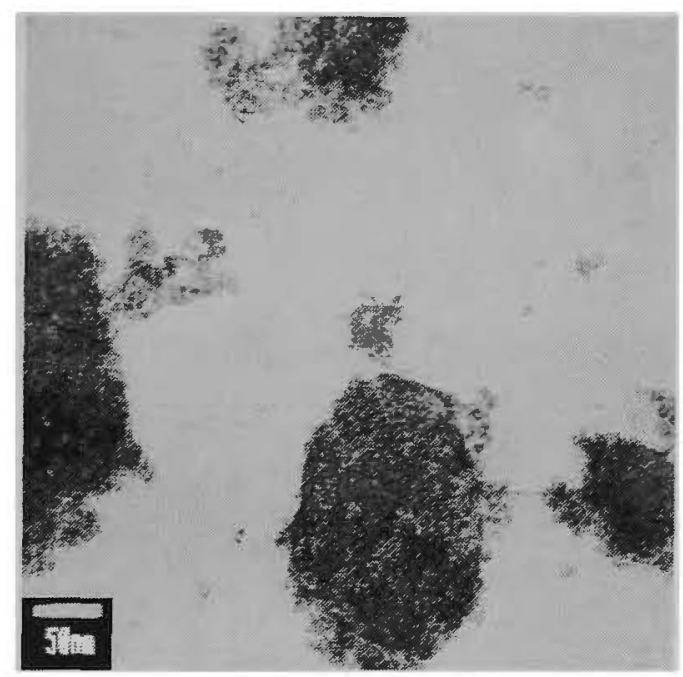

(c)

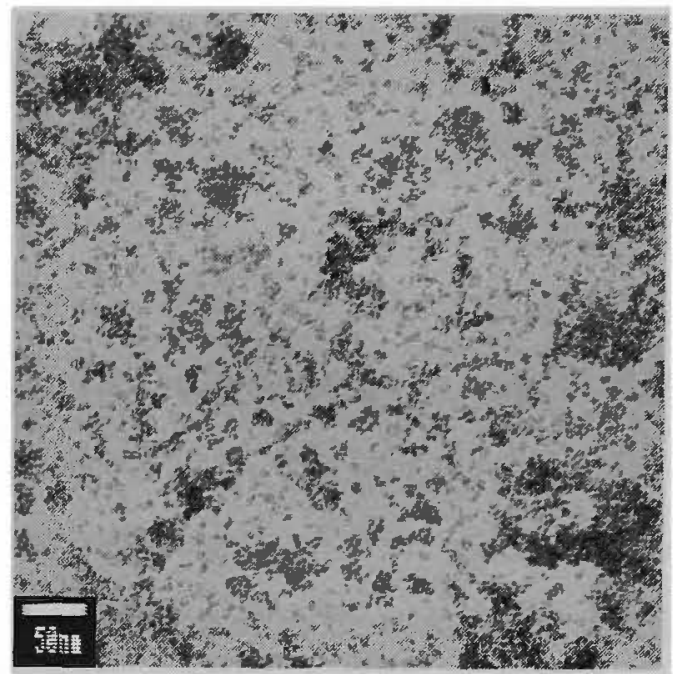

(b)

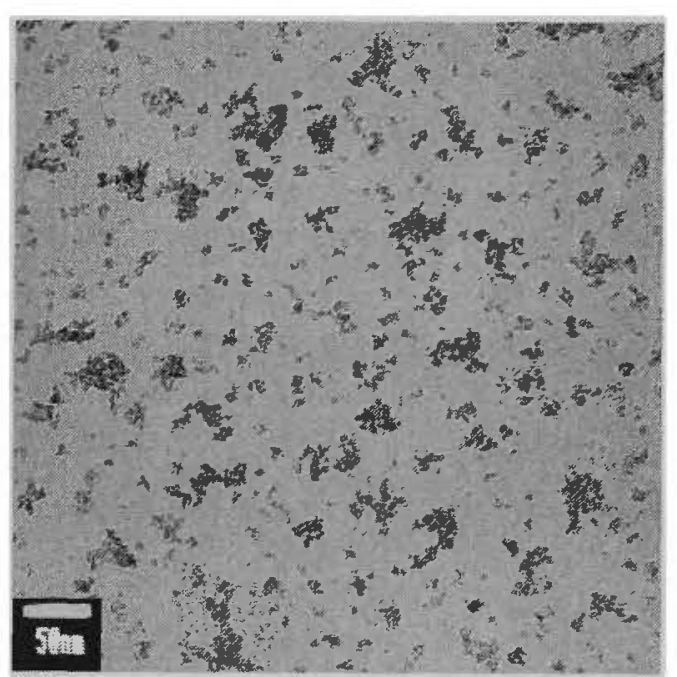

(d)

Figure 5.5 : Bright field TEM images of milled $\mathrm{ZrCl}_{4}+2 \mathrm{MgO}$ following (a) washing, (b) heat-treatment and washing, (c) washing and methanol rinsing, and (d) heattreatment, washing, and methanol rinsing.

Table 5.3 shows the XRD and BET crystallite sizes of the dried and calcined $\mathrm{ZrO}_{2}$ powders prepared using various post-milling treatments. The XRD and BET crystallite sizes show a close correspondence for those powders prepared with a post-milling heat treatment. Powders prepared without post-milling heat treatment showed XRD 
crystallite sizes that were substantially larger than the BET particle size, which suggests that the agglomerates were porous.

\section{(5.2.3) Effect of Dilution and Milling Duration}

The gradual reaction mechanism of the $\mathrm{ZrCl}_{4}+2 \mathrm{MgO}$ system suggests that ultrafine $\mathrm{ZrO}_{2}$ powders could be synthesized by milling the precursors for a short duration and then driving the reaction to completion by a subsequent low temperature heat-treatment. This possibility was confirmed by milling $\mathrm{ZrCl}_{4}+2 \mathrm{MgO}$ reactant mixtures for times ranging from 10 minutes up to 6 hours and then heat-treating at $500^{\circ} \mathrm{C}$ for 1 hour. Figure 5.6 shows the XRD patterns of the washed $\mathrm{ZrO}_{2}$ powders. All of the patterns correspond to tetragonal $\mathrm{ZrO}_{2}$. The peak breadth increased with the milling time up to a steady-state value after approximately 3 hours, indicating a decrease in crystallite size with milling time. Similar behaviour was observed for reactant mixtures containing $\mathrm{MgCl}_{2}$ diluent.

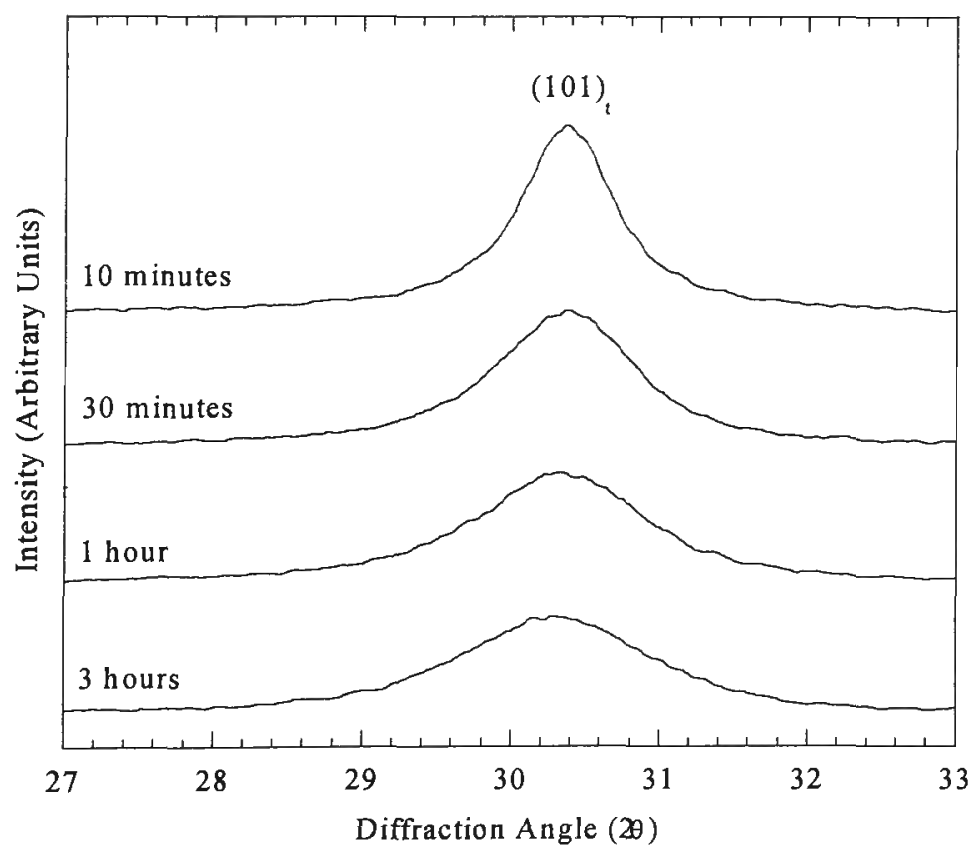

Figure 5.6 : XRD patterns of $\mathrm{ZrO}_{2}$ synthesised from $\mathrm{ZrCl} 4+2 \mathrm{MgO}$ using milling times ranging from 10 minutes to 3 hours.

Figure 5.7 shows the $\mathrm{XRD}$ and $\mathrm{BET}$ crystallite size of washed $\mathrm{ZrO}_{2}$ powders synthesised from $\mathrm{ZrCl}_{4}+2 \mathrm{MgO}$ as a function of the milling time. To ensure complete 
reaction of the precursors, the as-milled powders were heat-treated at $500^{\circ} \mathrm{C}$ for 1 hour prior to washing. For all powders, the XRD and BET crystallite sizes are in close agreement, which indicates that the powders largely consist of dispersed single crystal particles. The size of these particles decreased with milling time down to steady state value. The achievement of this steady-state particle size approximately coincides with the minimum time required for complete reaction of the precursors during milling.

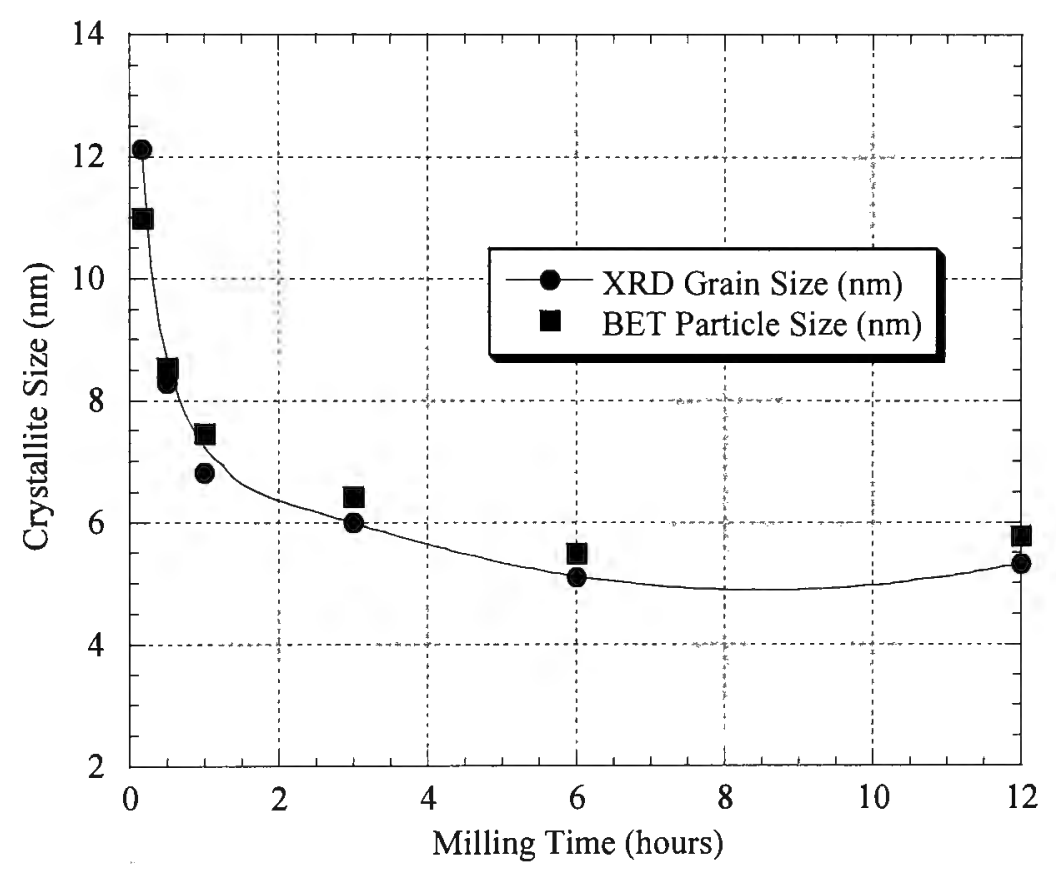

Figure 5.7 : $\mathrm{XRD}$ and $\mathrm{BET}$ crystallite size as functions of milling time for $\mathrm{ZrCl}_{4}+$ $2 \mathrm{MgO}$.

The effect of dilution with $\mathrm{MgCl}_{2}$ on the average particle size of the $\mathrm{ZrO}_{2}$ product phase was examined: for $\mathrm{ZrCl}_{4}+2 \mathrm{MgO}$ reactant mixtures that were reacted to completion during milling. All powders were prepared using a milling duration of 6 hours and a post-milling heat treatment of $500^{\circ} \mathrm{C}$ for 1 hour. The purpose of the post-milling heattreatment was to inhibit chemical interaction with the wash water, thus ensuring that the measured particle size was representative of that formed during milling. The results of this study are shown in Figure 5.8, which shows the XRD and BET crystallite size of washed $\mathrm{ZrO}_{2}$ powders prepared from $\mathrm{ZrCl}_{4}+2 \mathrm{MgO}+\mathrm{xMgCl}_{2}$ reactant mixtures, where $\mathrm{x}=0,2,4$, and 6 . Although the addition of $\mathrm{MgCl}_{2}$ to the reactant mixture increased the volume fraction of salt in the reaction product from 0.79 to 0.94 there was no appreciable variation in the measured particle size. 


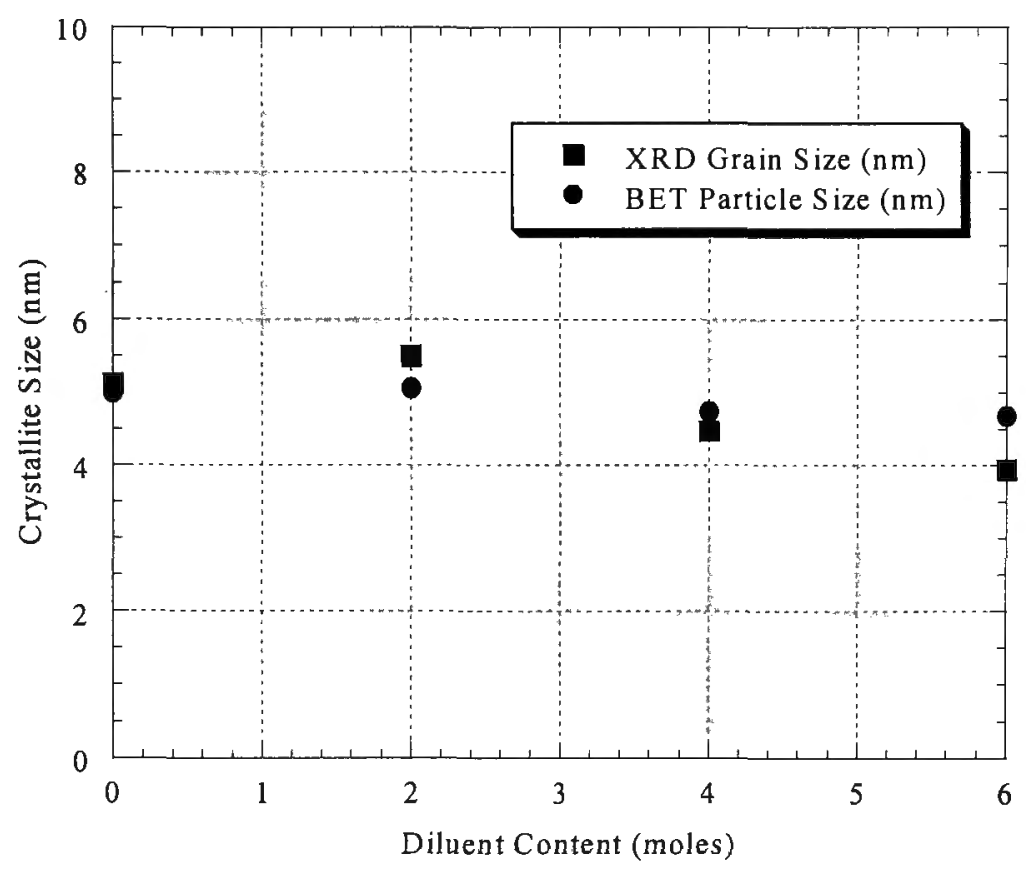

Figure 5.8 : Variation in BET and XRD crystallite size as functions of diluent content.

\section{(5.2.4) Effect of Heat Treatment}

Figure 5.9 shows the effect of post-milling heat treatment temperature and duration on the specific surface area of the washed $\mathrm{ZrO}_{2}$ powder. At temperatures below the melting point of the $\mathrm{MgCl}_{2}$ matrix phase $\left(714^{\circ} \mathrm{C}\right)$, the specific surface area initially increased before reaching a maximum and then slowly declined with increasing heat treatment duration. The initial increase can be attributed to a decrease in hard agglomerate formation arising from the reduced reactivity of heat treated $\mathrm{ZrO}_{2}$ towards the wash water, as mentioned previously. Above the melting point of $\mathrm{MgCl}_{2}$, the specific surface area showed an initially rapid decrease followed by a gradual decline. 


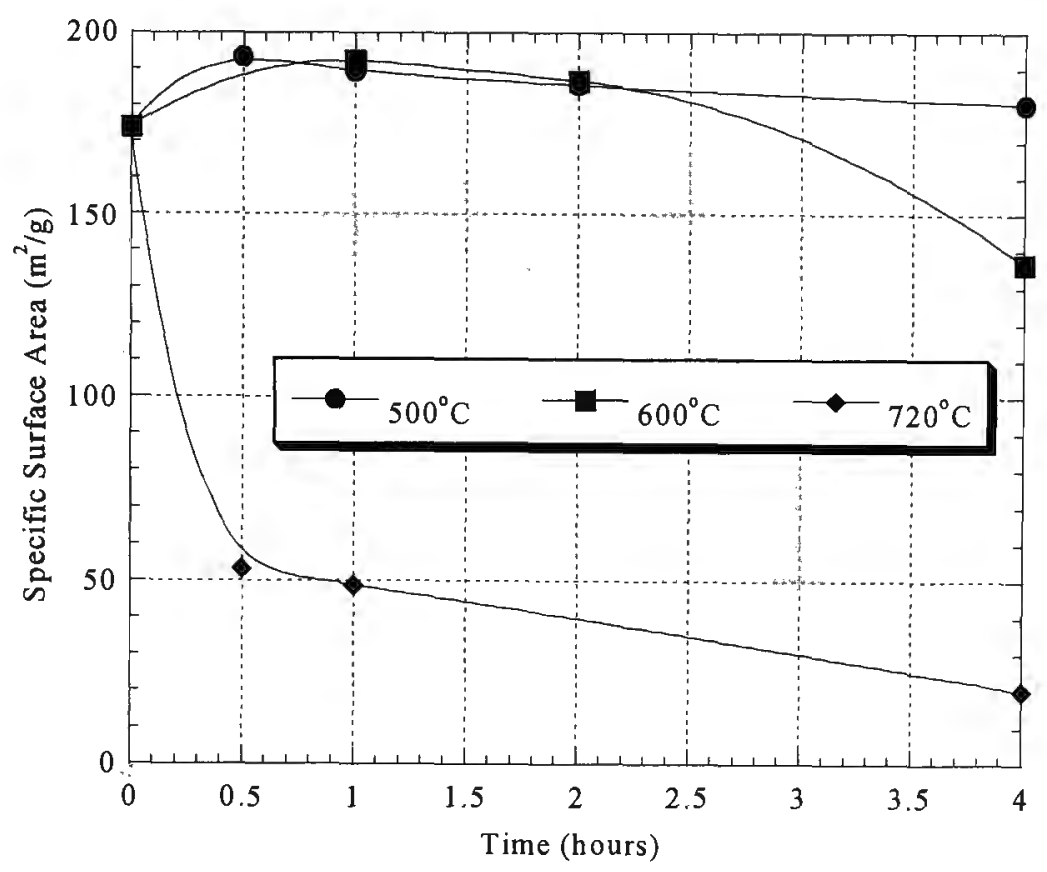

Figure 5.9 : BET surface area of washed $\mathrm{ZrO}_{2}$ powder as a function of post-milling heat treatment temperature and duration.

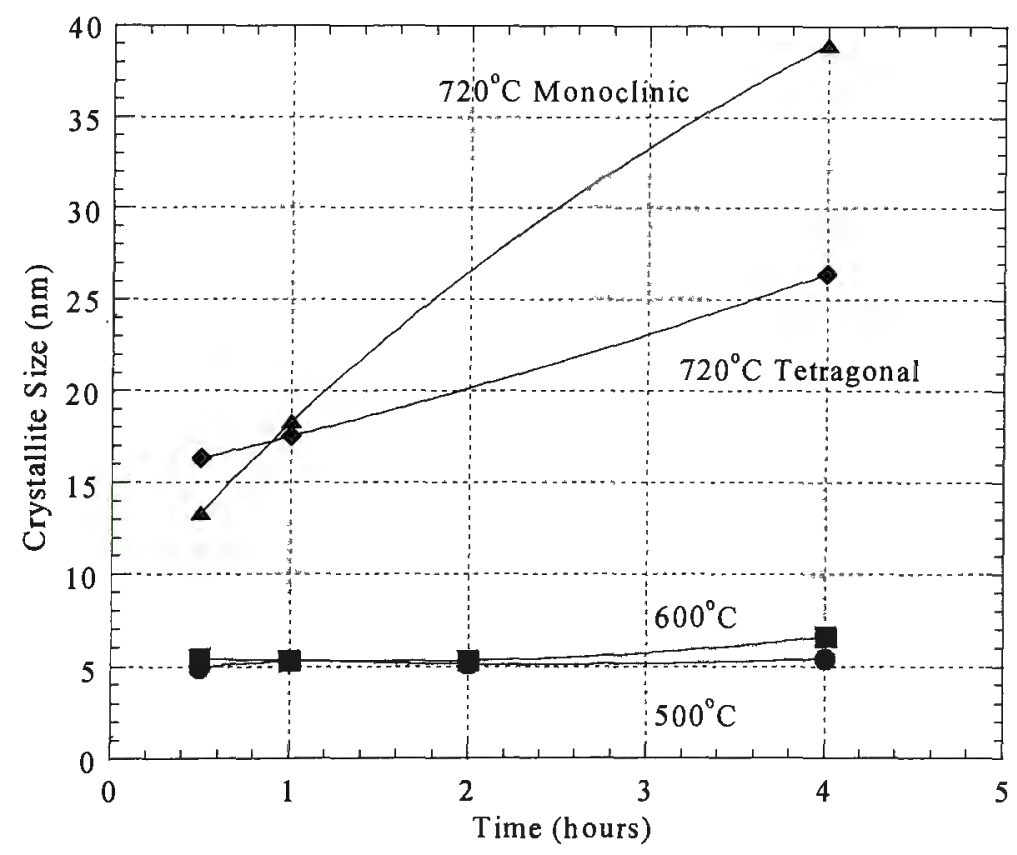

Figure 5.10 : $\mathrm{XRD}$ crystallite size of washed $\mathrm{ZrO}_{2}$ powder as a function of post-milling heat treatment temperature and duration. 


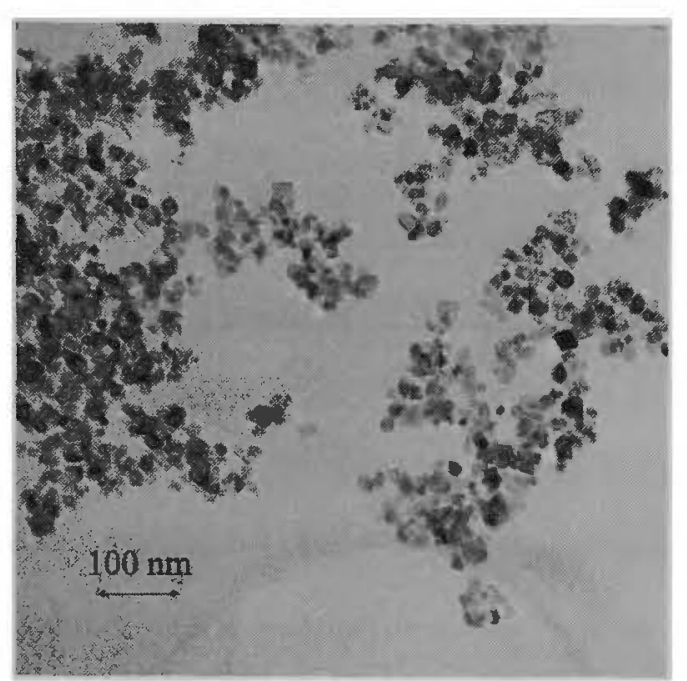

(a)

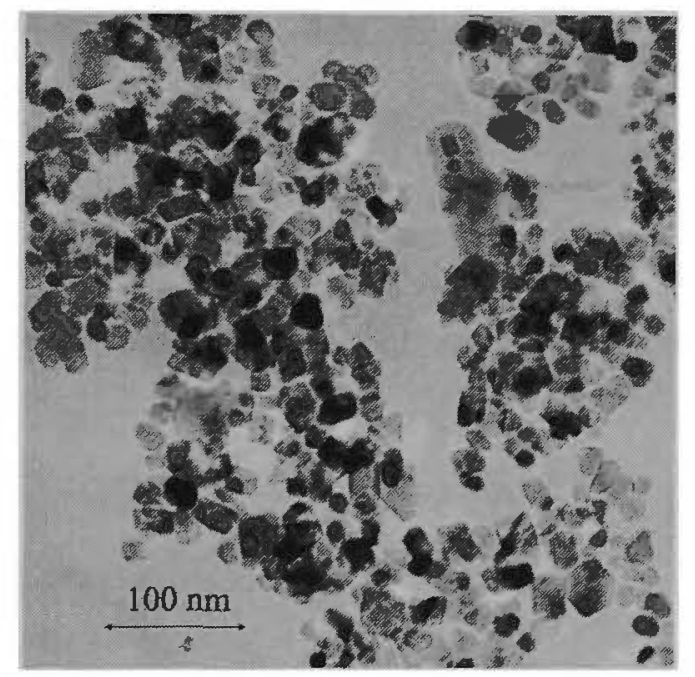

(b)

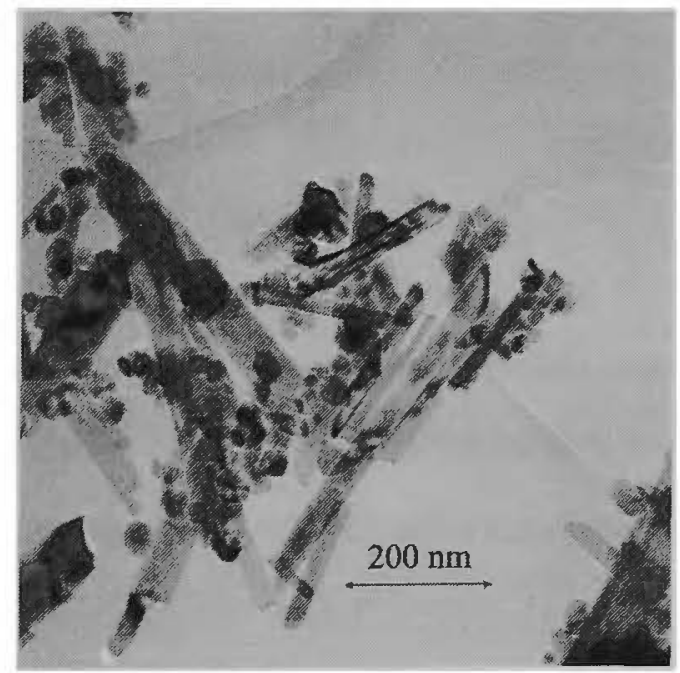

(c)

Figure 5.11 : Bright field TEM images of washed $\mathrm{ZrO}_{2}$ prepared from milled $\mathrm{ZrCl}_{4}+$ $2 \mathrm{MgO}$ using a post-milling heat treatment of $720^{\circ} \mathrm{C}$ for (a) 30 minutes, (b) 1 hour, and (c) 4 hours. 
Figure 5.10 shows the variation in $\mathrm{XRD}$ crystallite size of washed $\mathrm{ZrO}_{2}$ powder as a function of the post-milling heat treatment temperature and duration. Consistent with the variation in specific surface area shown in Figure 5.9, the crystallite size remained largely unchanged at temperature below the melting point of the $\mathrm{MgCl}_{2}$ matrix phase. The tetragonal structure of the $\mathrm{ZrO}_{2}$ also remained unaltered. In contrast, heat treatment at $720^{\circ} \mathrm{C}$ caused significant crystallite growth and partial transformation of the initially tetragonal $\mathrm{ZrO}_{2}$ to the equilibrium monoclinic structure. Both monoclinic and tetragonal crystallites show a near linear increase in size with the duration of heat treatment between 0.5 and 4 hours.

Examination by TEM of the powders prepared using heat-treatment temperatures of 500 and $600^{\circ} \mathrm{C}$ revealed no readily apparent change to either the particle size or morphology. In contrast, as shown in Figure 5.11 (a), heat-treatment at $720^{\circ} \mathrm{C}$ for 0.5 hours resulted in significant coarsening of the powder particles. Continued heat-treatment resulted in further particle growth and ultimately the appearance of acicular particles with a large aspect ratio.

\section{(5.3) Lithium Oxide Reactions}

\section{(5.3.1) $\mathrm{ZrO}_{2}$ Synthesis}

Reaction of $\mathrm{ZrCl}_{4}$ with $\mathrm{Li}_{2} \mathrm{O}$ to form $\mathrm{ZrO}_{2}$ and $\mathrm{LiCl}$ is characterised by a large free energy change of $-561 \mathrm{~kJ} / \mathrm{mole}$, which indicates a strong driving force for chemical reaction. Despite this strong driving force, milling of the precursors did not result in chemical reaction to any measurable extent. As shown by XRD in Figure 5.12, milling of the $\mathrm{ZrCl}_{4}+2 \mathrm{Li}_{2} \mathrm{O}+\mathrm{xLiCl}$ reactant mixtures merely resulted in amorphisation of the $\mathrm{ZrCl}_{4}$ reactant phase and overall microstructural refinement.

Although accurate quantitative analysis of the peak broadening was difficult because of the mechanically induced structural disorder, it appears that following 6 hours of milling, the grain size of the crystalline $\mathrm{LiCl}$ and $\mathrm{Li}_{2} \mathrm{O}$ phases was constant at approximately $21 \mathrm{~nm}$ irrespective of the diluent content. 
Chemical reaction of the precursors, with the consequent formation of $\mathrm{ZrO}_{2}$ and $\mathrm{LiCl}$, was found to occur during post-milling heat treatment. For the undiluted reactant mixture, the mechanism of this thermally activated reaction was found to depend on the heating rate. This is illustrated in Figure 5.13, which shows DTA traces of milled $\mathrm{ZrCl}_{4}$ $+2 \mathrm{Li}_{2} \mathrm{O}$ reactant mixtures using heating rates of 20 and $80^{\circ} \mathrm{C} /$ minute. The trace obtained using the lower heating rate exhibits several exothermic events, indicating that chemical reaction of the precursors occurred in multiple stages. In contrast, the trace obtained using the higher heating rate exhibits a solitary exothermic peak. The magnitude and sloped appearance of this peak indicates that the higher heating rate resulted in combustive reaction of the precursors.

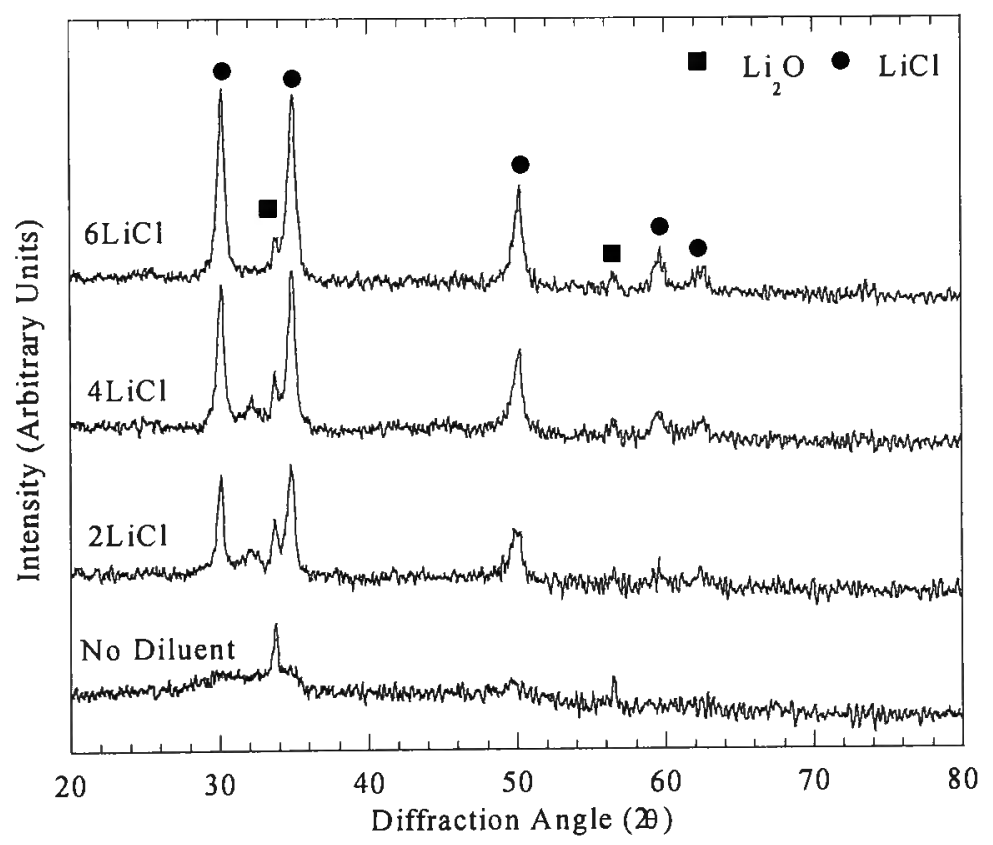

Figure 5.12 : XRD patterns of $\mathrm{ZrCl}_{4}+2 \mathrm{Li}_{2} \mathrm{O}+\mathrm{xLiCl}$ reactant mixtures following milling for 6 hours.

XRD analysis was used to follow the chemical evolution of the milled $\mathrm{ZrCl}_{4}+2 \mathrm{Li}_{2} \mathrm{O}$ reactant mixture during slow heating. Samples were heated in the DTA at either 20 or $80^{\circ} \mathrm{C} /$ minute up to the set temperature and then air quenched to room temperature. Figure 5.14 shows the XRD patterns of these heat-treated samples. Heating of the asmilled powder to $150^{\circ} \mathrm{C}$ resulted in the appearance of broad diffraction peaks corresponding to $\mathrm{LiCl}$. Peaks corresponding to $\mathrm{Li}_{2} \mathrm{ZrCl}_{6}$ appeared following heating to $300^{\circ} \mathrm{C}$. By $500^{\circ} \mathrm{C}$, the peaks corresponding to the $\mathrm{Li}_{2} \mathrm{O}$ reactant and the $\mathrm{Li}_{2} \mathrm{ZrCl}_{6}$ 
intermediate product phase had disappeared and were replaced by peaks corresponding to the final product phases of $\mathrm{LiCl}$ and tetragonal $\mathrm{ZrO}_{2}$.

The volume of sample produced by heat-treatment in the DTA was insufficient to allow recovery of enough $\mathrm{ZrO}_{2}$ powder by washing for subsequent analysis. Attempts at overcoming this limitation by heat-treating bulk quantities of the as-milled powder failed. Rather than undergoing multi-stage reaction, the as-milled powder underwent combustion irrespective of the applied heating rate. As a result, no definitive data regarding the phase content or crystallite size was obtained. However, close examination of Figure 5.14 suggests that the $\mathrm{ZrO}_{2}$ phase was predominantly tetragonal and exhibited significant peak broadening.

Figure 5.15 shows the $\mathrm{XRD}$ pattern of $\mathrm{ZrCl}_{4}+2 \mathrm{Li}_{2} \mathrm{O}$ following (a) milling for 6 hours, (b) heat-treatment at $400^{\circ} \mathrm{C}$ using a rapid heating ramp and a hold of 1 hour, and (c) subsequent washing. Heat-treatment of the as-milled powder using rapid heating resulted in the formation of $\mathrm{LiCl}$ and monoclinic $\mathrm{ZrO}_{2}$.

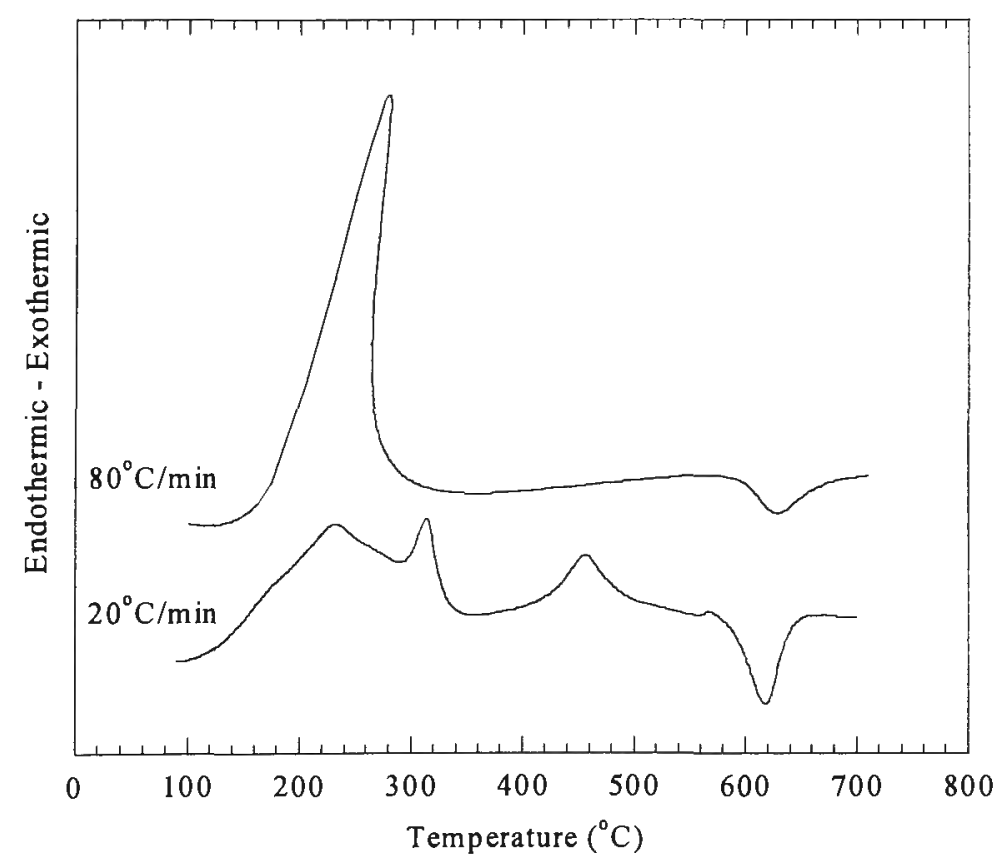

Figure 5.13 : DTA traces of milled $\mathrm{ZrCl}_{4}+2 \mathrm{Li}_{2} \mathrm{O}$ reactant mixtures using heating rates of 20 and $80^{\circ} \mathrm{C} /$ minute. 


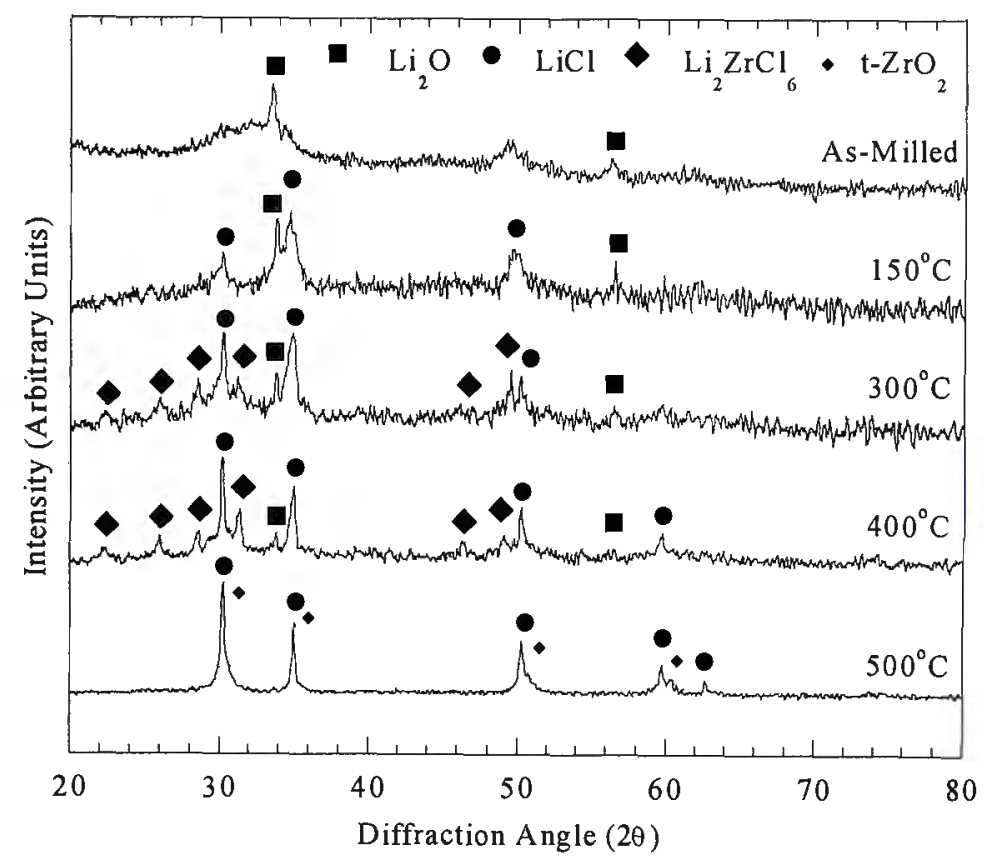

Figure 5.14 : XRD patterns of milled $\mathrm{ZrCl}_{4}+2 \mathrm{Li}_{2} \mathrm{O}$ following heat-treatment at various temperatures in the DTA using a heating rate of $20^{\circ} \mathrm{C} /$ minute.

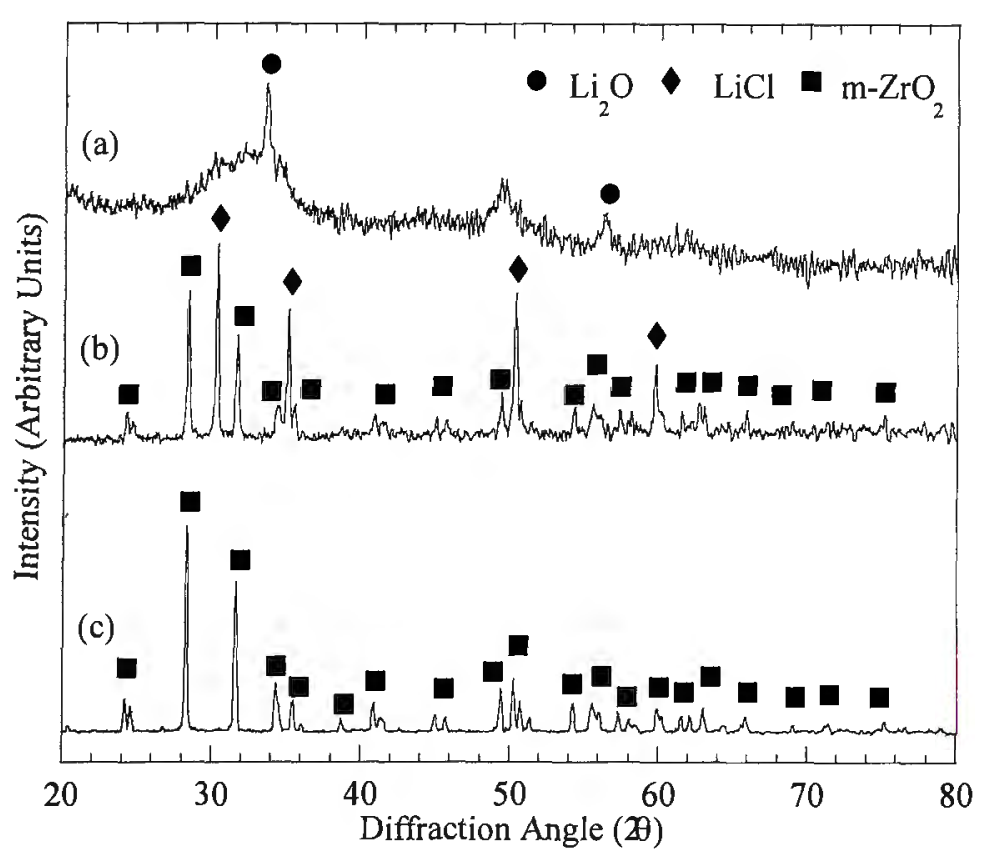

Figure 5.15 : XRD pattern of $\mathrm{ZrCl}_{4}+2 \mathrm{Li}_{2} \mathrm{O}$ following (a) milling for 6 hours, (b) heattreatment at $400^{\circ} \mathrm{C}$ using a rapid heating ramp and a hold of 1 hour, and (c) subsequent washing. 
Figure 5.16 shows DTA traces of $\mathrm{ZrCl}_{4}+2 \mathrm{Li}_{2} \mathrm{O}+\mathrm{xLiCl}$ reactant mixtures that were obtained using a heating rate of $80^{\circ} \mathrm{C} /$ minute. All of the traces exhibit an exothermic peak corresponding to chemical reaction of the precursors followed by an endothermic peak corresponding to melting of the $\mathrm{LiCl}$ by-product phase. The addition of $2 \mathrm{LiCl}$ to the undiluted reactant mixture raised the initiation temperature for chemical reaction by approximately $230^{\circ} \mathrm{C}$. Further additions of diluent beyond $2 \mathrm{LiCl}$ had negligible effect on the initiation temperature but did further decrease the magnitude of the reaction peak.

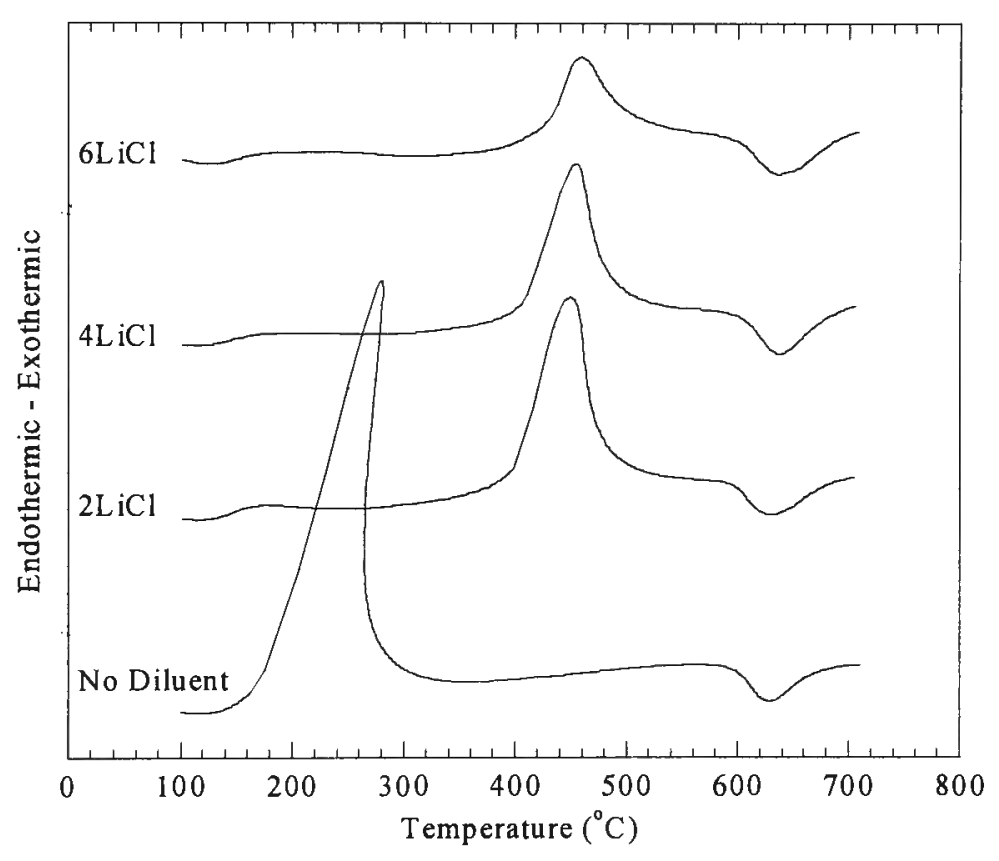

Figure 5.16 : DTA traces of milled $\mathrm{ZrCl}_{4}+2 \mathrm{Li}_{2} \mathrm{O}+\mathrm{xLiCl}$ reactant mixtures using a heating rate of $80^{\circ} \mathrm{C} /$ minute.

Figure 5.17 shows the $\mathrm{XRD}$ patterns of washed $\mathrm{ZrO}_{2}$ powders synthesised from $\mathrm{ZrCl}_{4}+$ $2 \mathrm{Li}_{2} \mathrm{O}+\mathrm{xLiCl}$ reactant mixtures using a rapid heating rate and a hold of 1 hour at $400^{\circ} \mathrm{C}$. The pattern of the powder synthesised from the undiluted reactant mixture $(\mathrm{x}=$ 0 ) consists solely of diffraction peaks corresponding to the monoclinic phase. In contrast, the patterns of the powders synthesised from the diluted reactant mixtures contain peaks corresponding to both the monoclinic and tetragonal phases. The relative intensity of the tetragonal peak to the monoclinic increased with the concentration of diluent in the initial reactant mixture. This was also accompanied by increased peak broadening, which indicates a decrease in the crystallite size with higher levels of dilution. 


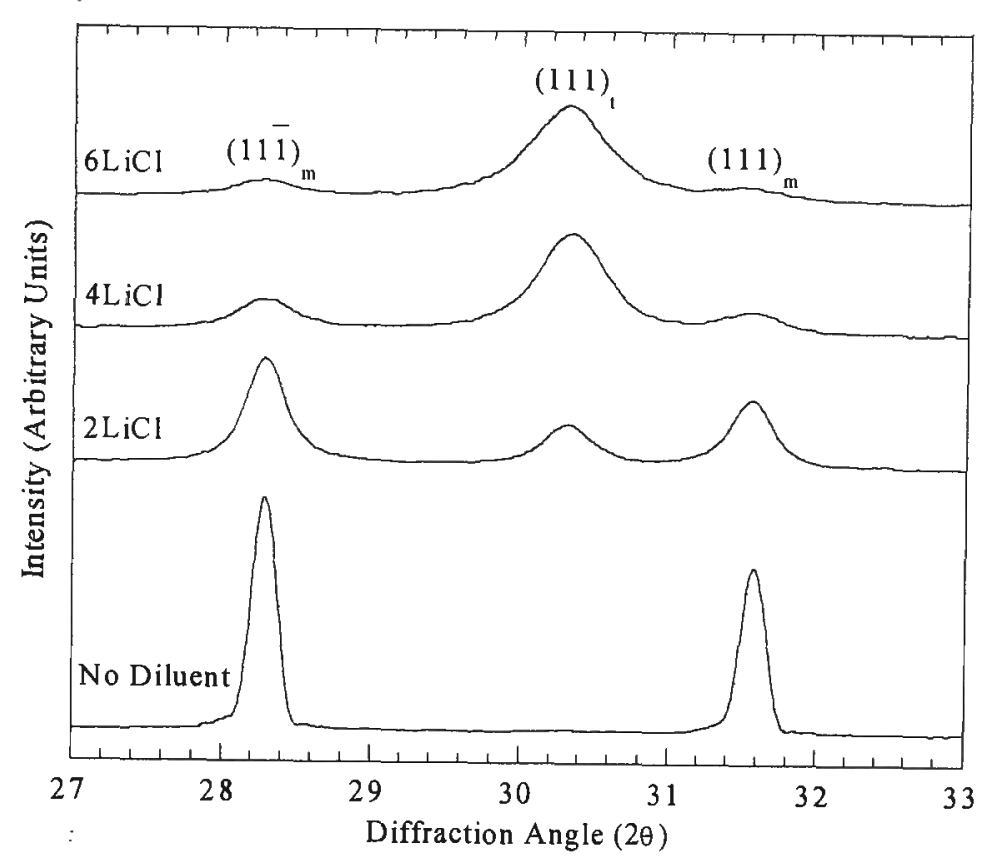

Figure 5.17 : XRD patterns of washed $\mathrm{ZrO}_{2}$ synthesised from $\mathrm{ZrCl}_{4}+2 \mathrm{Li}_{2} \mathrm{O}+\mathrm{xLiCl}$ reactant mixtures.

\section{(5.3.2) Synthesis of Stabilised Zirconia}

Reactant systems for the synthesis of $\mathrm{MgO}\left(\mathrm{MgCl}_{2}+\mathrm{Li}_{2} \mathrm{O}\right)$ and $\mathrm{Y}_{2} \mathrm{O}_{3}\left(2 \mathrm{YCl}_{3}+3 \mathrm{Li}_{2} \mathrm{O}\right)$ were found to exhibit similar chemical evolution during processing to that observed previously for $\mathrm{ZrCl}_{4}+2 \mathrm{Li}_{2} \mathrm{O}$. Milling of the precursors resulted in amorphisation of the chloride reactant and overall microstructural refinement. Chemical reaction, with the consequent formation of oxide and $\mathrm{LiCl}$, only occurred during post-milling heattreatment. Subsequent washing with appropriate solvents was successful in recovering $\mathrm{MgO}$ and $\mathrm{Y}_{2} \mathrm{O}_{3}$ powders. $\mathrm{MgO}$ is slightly soluble in water and was therefore washed with methanol.

The only notable difference in the reaction behaviour of these two systems compared to $\mathrm{ZrCl}_{4}+2 \mathrm{Li}_{2} \mathrm{O}$ was their insensitivity to the applied heating rate. Both systems reacted combustively during post-milling heat-treatment regardless of the heating rate. Thermal analysis using DTA showed that both the $\mathrm{MgO}$ and $\mathrm{Y}_{2} \mathrm{O}_{3}$ synthesis reactions initiated at approximately $220^{\circ} \mathrm{C}$. 
This similarity in processing behaviour suggests that Mg-PSZ and Y-TZP powders could be synthesised by simply combining the basic $\mathrm{ZrO}_{2}$ synthesis reaction with that for the stabiliser. This possibility was confirmed by experiments with the reaction systems given by equations (1) and (2).

$$
\begin{aligned}
& 0.1 \mathrm{MgCl}_{2}+0.9 \mathrm{ZrCl}_{4}+1.9 \mathrm{Li}_{2} \mathrm{O} \rightarrow(\mathrm{MgO})_{0.1}\left(\mathrm{ZrO}_{2}\right)_{0.9}+3.6 \mathrm{LiCl} \\
& 0.06 \mathrm{YCl}_{3}+0.97 \mathrm{ZrCl}_{4}+2.03 \mathrm{Li}_{2} \mathrm{O} \rightarrow\left(\mathrm{Y}_{2} \mathrm{O}_{3}\right)_{0.03}\left(\mathrm{ZrO}_{2}\right)_{0.97}+4.06 \mathrm{LiCl}
\end{aligned}
$$

These systems were found to undergo reaction in a manner essentially identical to the basic $\mathrm{ZrO}_{2}$ synthesis system. Undiluted reactant mixtures underwent multistage reaction for slow heating rates and reacted combustively for rapid heating rates. As shown in Figure 5.18, the initiation temperature for combustive reaction of the undiluted systems during rapid heating was highest for the Mg-PSZ reaction followed by the Y-TZP and lowest for the $\mathrm{ZrO}_{2}$ system. The initiation temperature for reaction of the diluted systems was the as that of the diluted $\mathrm{ZrO}_{2}$ synthesis systems.

The reaction systems given by equations (5.1) and (5.2) were found to successfully yield Mg-PSZ and Y-TZP solid solutions. This is demonstrated by an increase in the tetragonal volume fraction and also by a shift of the (111)t XRD peak, as illustrated in Figure 5.19, which shows the patterns of $\mathrm{ZrO}_{2}, \mathrm{Mg}-\mathrm{PSZ}$, and Y-TZP powders that were prepared from reactant mixtures containing $6 \mathrm{LiCl}$ diluent. Compared to the undoped $\mathrm{ZrO}_{2}$, the peak is shifted to a lower angle for the Y-TZP and to a higher angle for the Mg-PSZ. 


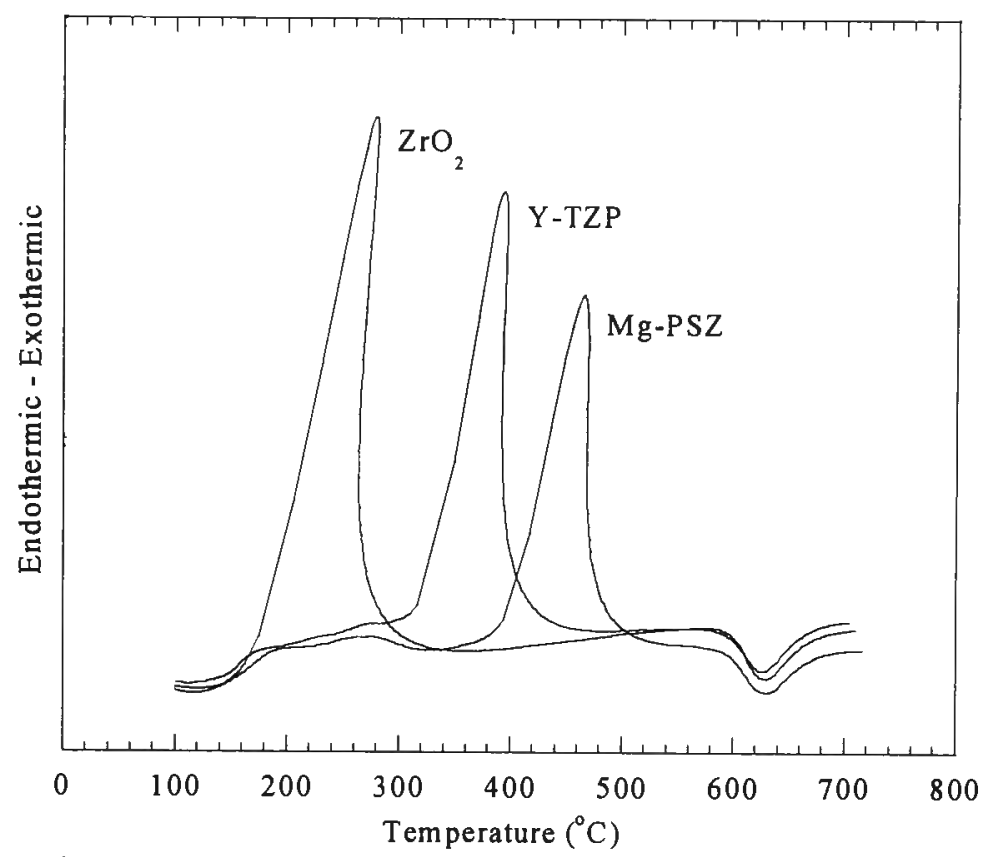

Figure 5.18 : DTA traces of milled reactant mixtures for the synthesis of $\mathrm{ZrO}_{2}$, Y-TZP, and Mg-PSZ.

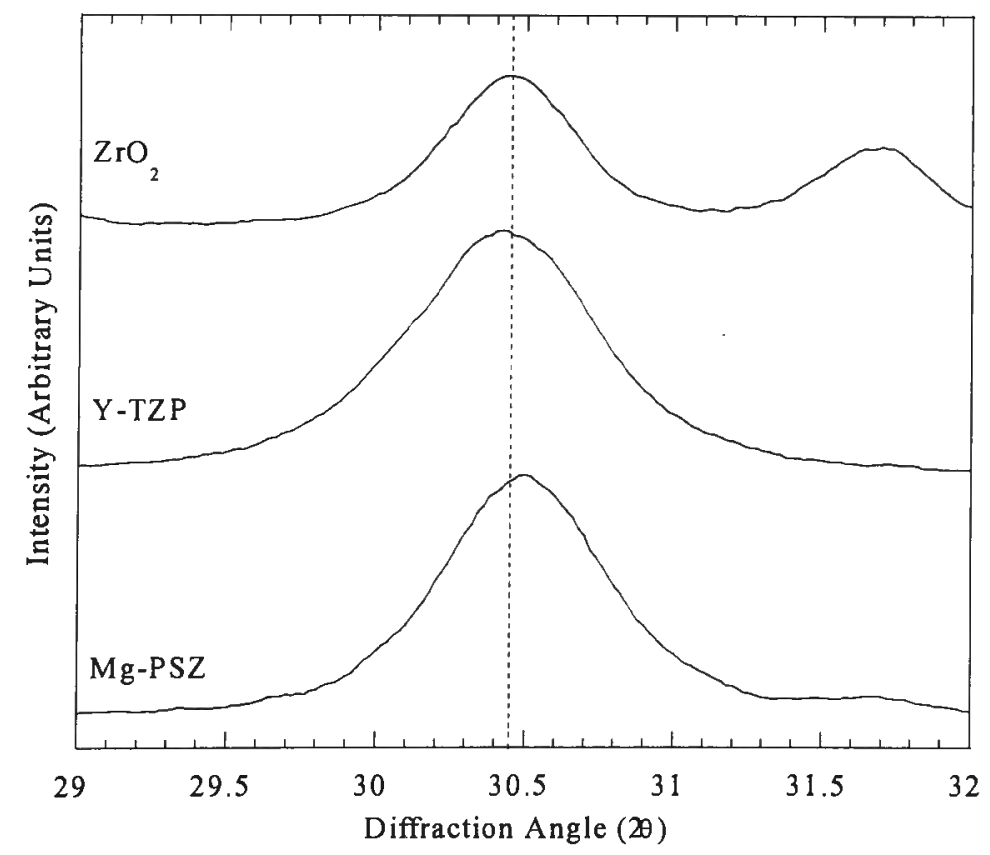

Figure 5.19 : XRD patterns showing the (111) diffraction peak of $\mathrm{ZrO}_{2}, \mathrm{Y}-\mathrm{TZP}$, and Mg-PSZ powders that were synthesized from reactant mixtures containing $6 \mathrm{LiCl}$ diluent. 


\section{(5.3.3) Effect of Dilution and Milling Duration}

The effect of milling time on the average particle and crystallite size of the final washed powder was found to depend on the presence of $\mathrm{LiCl}$ diluent. The average particle and crystallite size produced by undiluted reactant mixtures was essentially unaffected by the milling time. In contrast, the average particle and crystallite size was found to decrease with milling time down to a lower limit for the diluted reaction systems. This behaviour is illustrated in Figure 5.20, which shows the XRD and BET crystallite size as functions of milling time for $\mathrm{ZrO}_{2}$ powders that were synthesised from $\mathrm{ZrCl}_{4}+2 \mathrm{Li}_{2} \mathrm{O}$ $+6 \mathrm{LiCl}$. For short milling times, the particle size determined from BET measurements is significantly larger than the crystallite size derived from XRD measurements. The BET and XRD crystallite measurements converge at longer milling times.

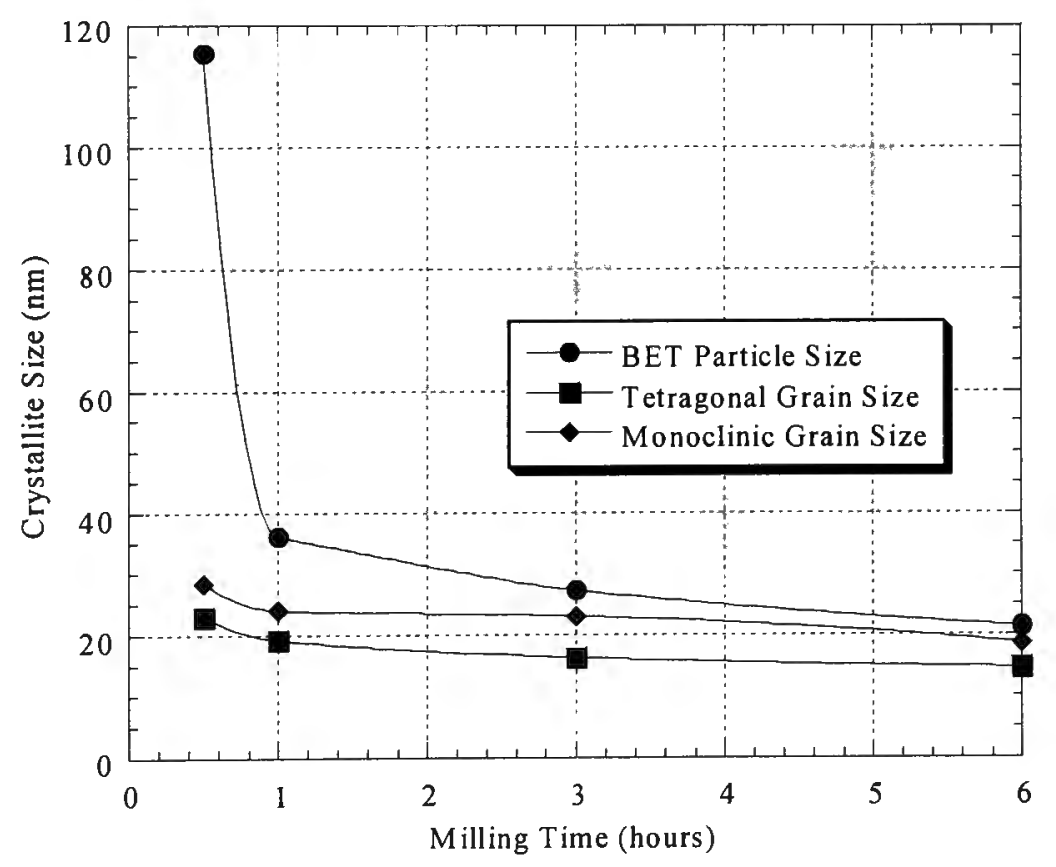

Figure 5.20 : $\mathrm{XRD}$ and $\mathrm{BET}$ crystallite size as functions of milling time for $\mathrm{ZrO}_{2}$ synthesised from $\mathrm{ZrCl}_{4}+2 \mathrm{Li}_{2} \mathrm{O}+6 \mathrm{LiCl}$.

The large difference between the BET particle size and the XRD crystallite size for powders prepared using short milling times indicates that these powders contain considerable hard agglomeration. This was confirmed by examination of the washed powders with TEM. As shown in Figure 5.21 (a), the $\mathrm{ZrO}_{2}$ powder that was prepared using a milling time of 30 minutes consists primarily of large aggregates of small 
crystallites. Increasing the milling time to 6 hours yielded a powder that consisted predominantly of dispersed single crystal particles, as shown in Figure 5.20 (b).

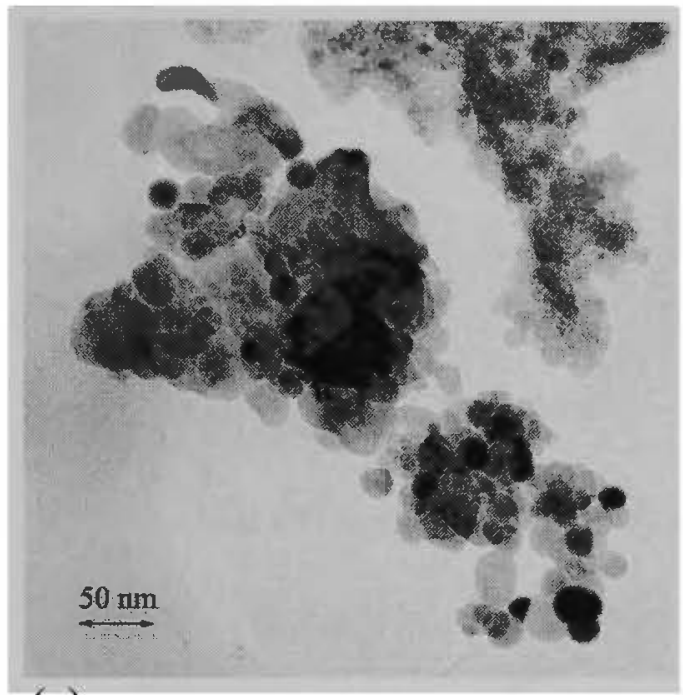

(a)

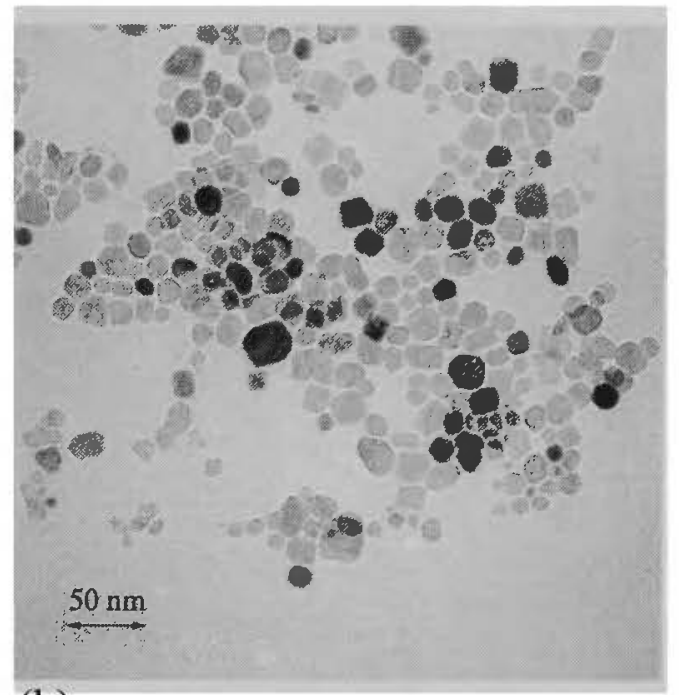

(b)

Figure 5.21 : Bright field TEM images of washed $\mathrm{ZrO}_{2}$ powders synthesised from $\mathrm{ZrCl}_{4}+2 \mathrm{Li}_{2} \mathrm{O}+6 \mathrm{LiCl}$ using milling times (a) 30 minutes and (b) 6 hours.

Figure 5.22 shows the XRD crystallite size, BET particle size, and phase content as functions of diluent content for the $\mathrm{ZrO}_{2}, \mathrm{Y}-\mathrm{TZP}$, and $\mathrm{Mg}-\mathrm{PSZ}$ synthesis systems. All three systems show a decrease in particle and crystallite size with higher levels of dilution. However, for low concentrations of diluent, the BET particle size is significantly larger than the XRD crystallite size. At high diluent concentrations the two measurements roughly coincide. For all levels of dilution, the tetragonal crystallite size is smaller than the monoclinic 
(a) $\mathrm{ZrCl}_{+}+2 \mathrm{Li}_{2} \mathrm{O}+\mathrm{xLiCl}$

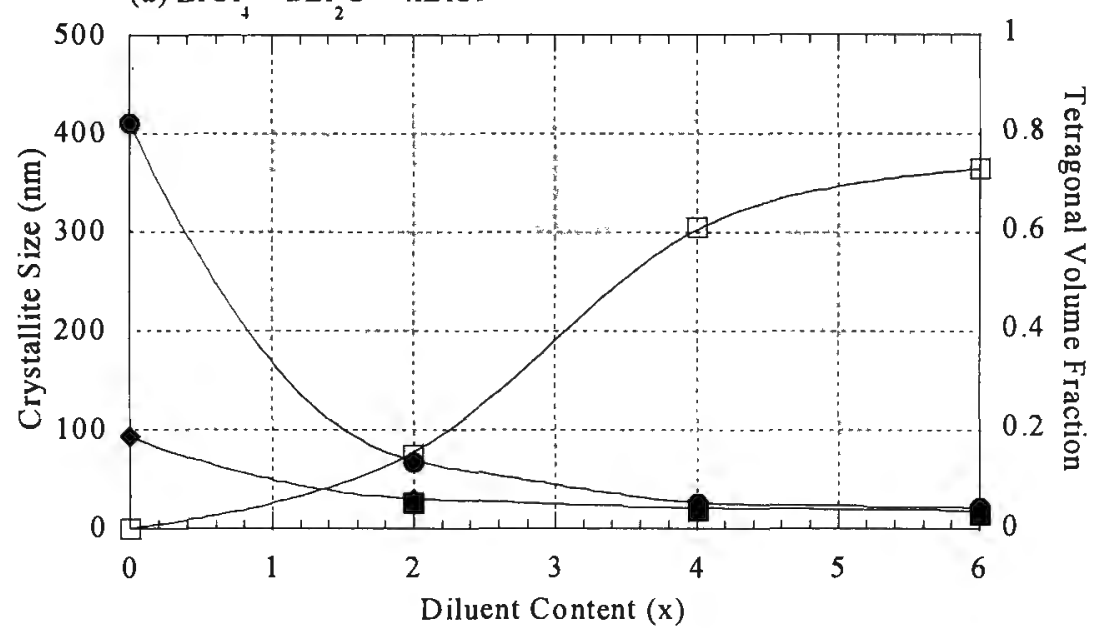

(b) $0.1 \mathrm{MgCl}_{2}+0.9 \mathrm{ZrCl}_{+}+1.9 \mathrm{Li}_{2} \mathrm{O}+\mathrm{xLiCl}$

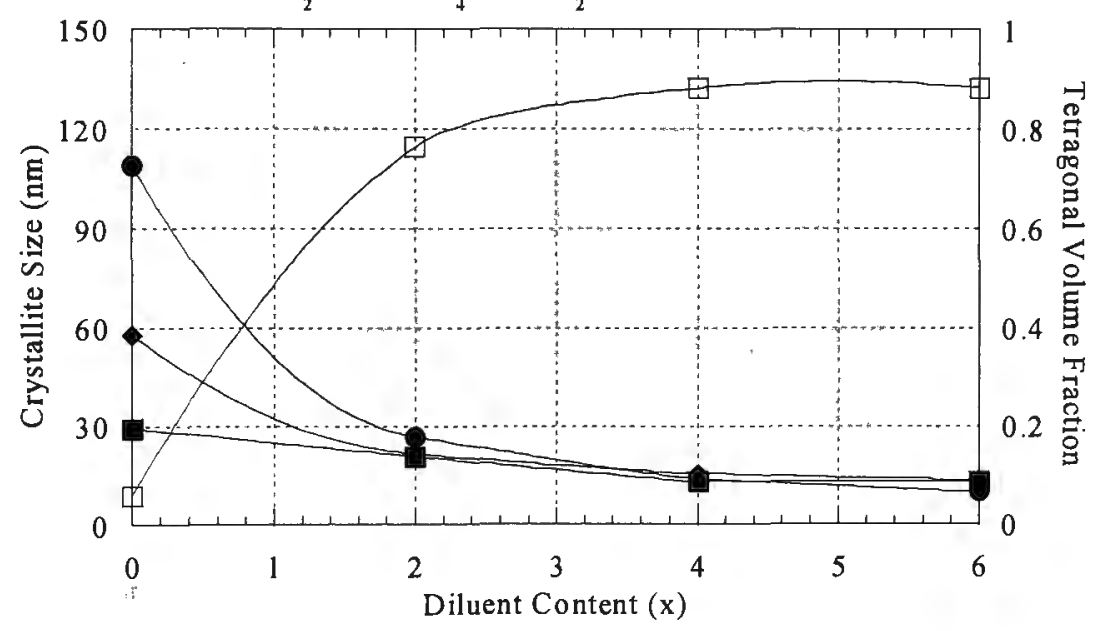

(c) $0.06 \mathrm{YCl}_{3}+0.97 \mathrm{ZrCl}_{+}+2.03 \mathrm{Li}_{2} \mathrm{O}+\mathrm{xLiCl}$

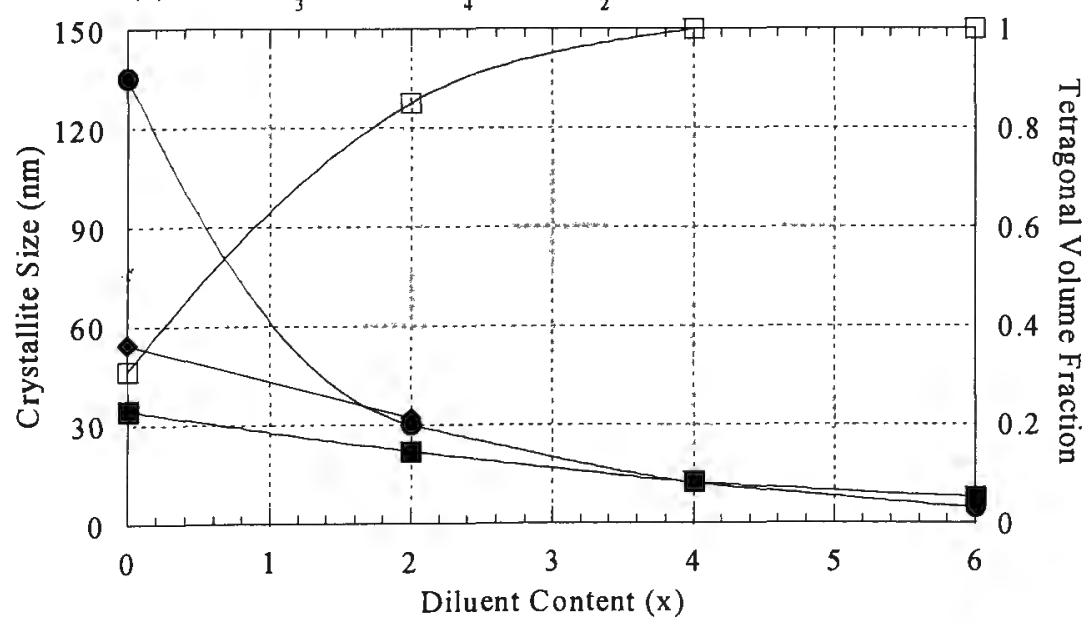

Figure 5.22 : BET particle size $(\bullet)$, monoclinic crystallite size $(\diamond)$, tetragonal crystallite size $(\boldsymbol{\square})$, and tetragonal volume fraction $(\boldsymbol{\square})$ as functions of diluent content for (a) $\mathrm{ZrO}_{2}$, (b) Mg-PSZ, and (c) Y-TZP. 


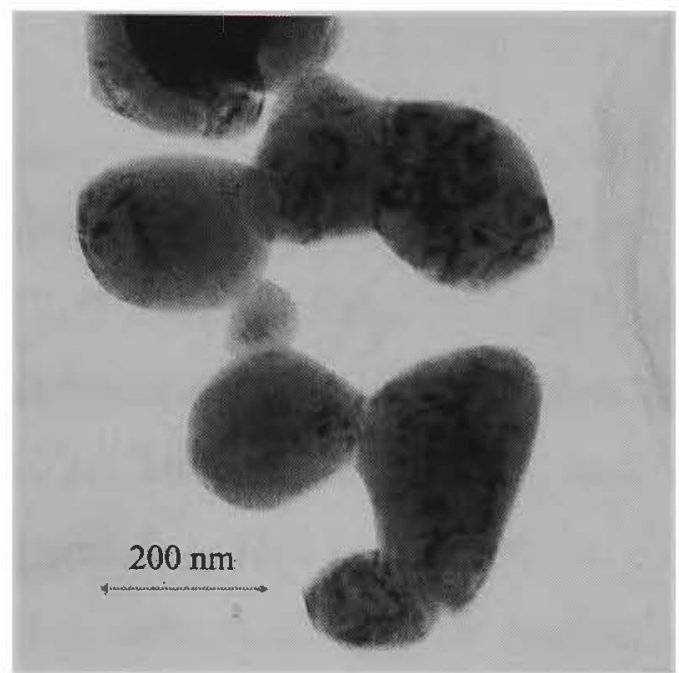

(a)

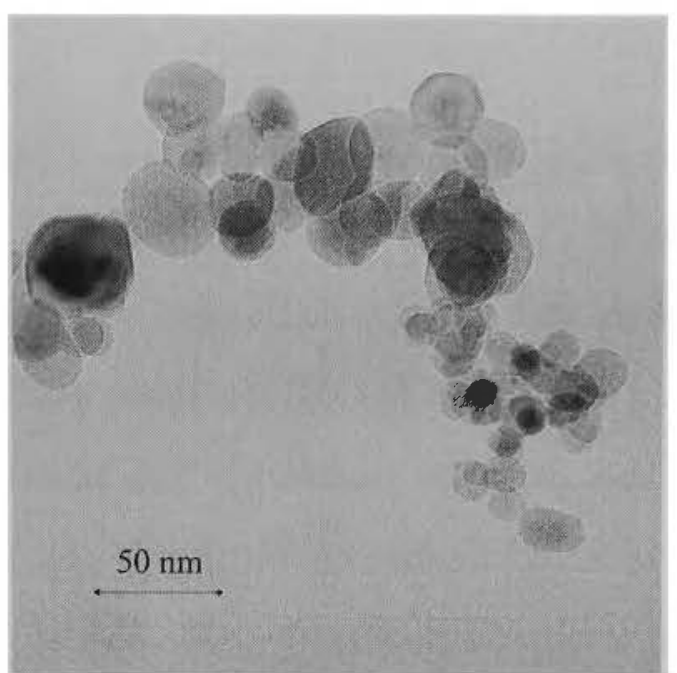

(b)

Figure 5.23 : Bright field TEM images of washed $\mathrm{ZrO}_{2}$ powders synthesised from reactant mixtures containing (a) no diluent and (b) $4 \mathrm{LiCl}$.

The discrepancy between the BET particle size and XRD crystallite size for powders synthesised from reactant mixtures that contained low concentrations of diluent suggests that these powders predominantly consist of polycrystalline particles. At high concentrations of diluent the two measurements converge, indicative of dispersed single crystal particles. This was confirmed by examination of the washed powders with TEM. As shown in Figure 5.23 (a), $\mathrm{ZrO}_{2}$ powder synthesised without diluent is comprised of irregularly shaped particles that consist of individual crystallites that are joined together. In contrast, powder synthesised from a reactant mixture containing $4 \mathrm{LiCl}$ appears to consist primarily of dispersed single crystal particles, as shown in Figure 5.23 (b).

For all three systems, the decrease in particle and crystallite size with dilution was accompanied by an increase in the tetragonal volume fraction. For the $\mathrm{ZrO}_{2}$ system, the tetragonal volume fraction increased in a sigmoidal fashion to an upper limit of approximately $73 \%$. The Mg-PSZ system showed a monotonic increase in tetragonal volume fraction with dilution up to approximately $88 \%$. The Y-TZP powders showed a similar monotonic variation and were fully tetragonal for diluent levels in excess of 2LiCl. 


\section{(5.3.4) Effect of Heat Treatment}

Heat-treatment of the reacted powders, which consisted of zirconia particles embedded within a matrix of $\mathrm{LiCl}$, was found to result in similar behaviour to that observed for the $\mathrm{ZrCl}_{4}+2 \mathrm{MgO}$ system. No significant particle or crystallite growth was observed for heat-treatment temperatures below the melting point of the $\mathrm{LiCl}$ reaction by-product $\left(610^{\circ} \mathrm{C}\right)$. However, in contrast, heat-treatment at $700^{\circ} \mathrm{C}$ resulted in rapid coarsening of the particles. This is illustrated in Figure 5.24, which shows the variation in specific surface area with time of washed $\mathrm{ZrO}_{2}, \mathrm{Y}-\mathrm{TZP}$, and Mg-PSZ powders that were prepared from reactant mixtures containing $6 \mathrm{LiCl}$ diluent. The specific surface area of all three powders showed an initially rapid decrease before leveling off at approximately $15 \mathrm{~m}^{2} / \mathrm{g}$.

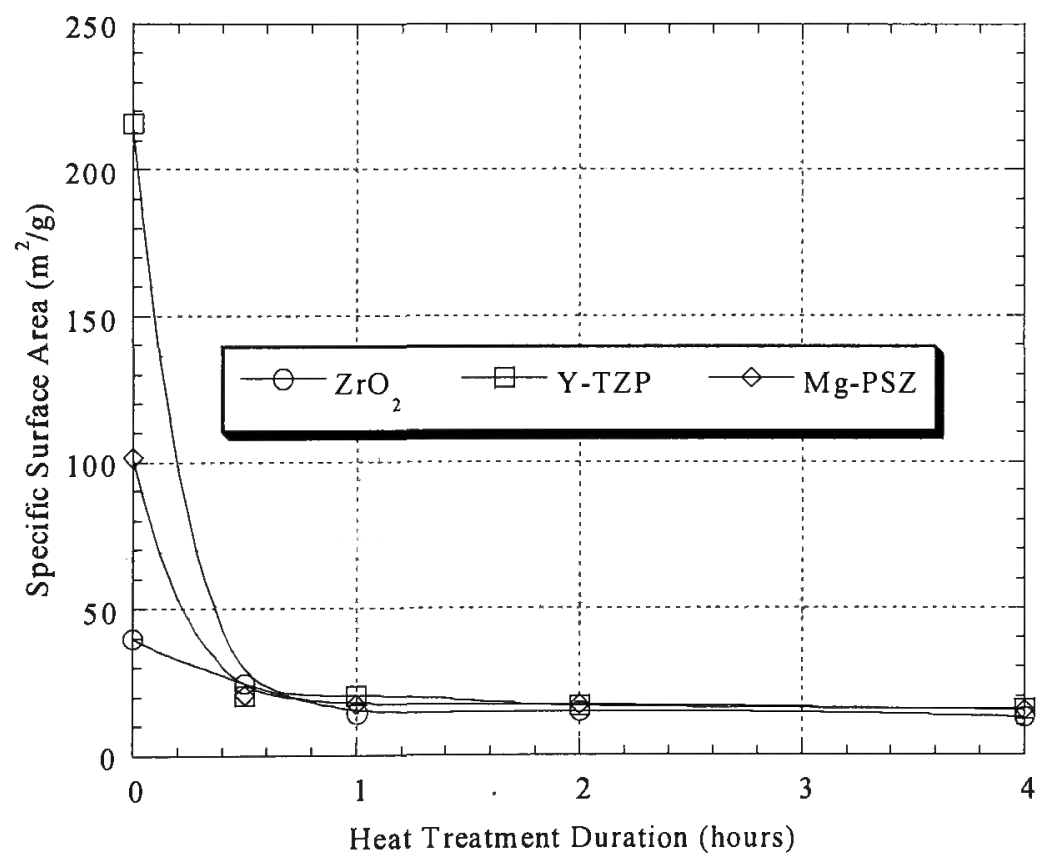

Figure 5.24 : Specific surface area as a function of heat-treatment duration for washed $\mathrm{ZrO}_{2}, \mathrm{Y}-\mathrm{TZP}$, and Mg-PSZ powders.

XRD analysis found that heat-treatment resulted in transformation of the powders to the equilibrium monoclinic structure and rapid crystallite growth. As illustrated in Figure 5.25 , which shows the monoclinic crystallite size as a function of heat-treatment duration, the crystallite size of the powders rapidly increased to a steady-state value of 
approximately $40 \mathrm{~nm}$, irrespective of their chemical composition and initial crystallite size.

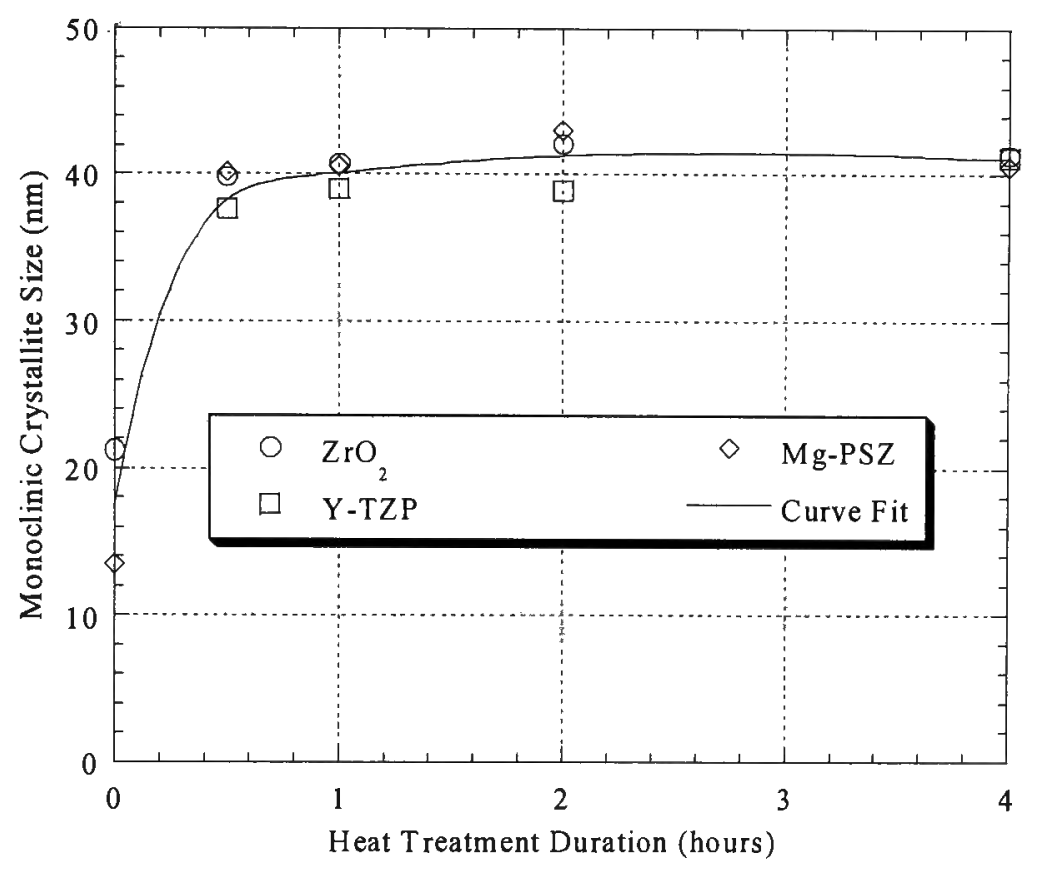

Figure 5.25 : Monoclinic crystallite size as a function of heat-treatment duration for washed $\mathrm{ZrO}_{2}, \mathrm{Y}-\mathrm{TZP}$, and Mg-PSZ powders.

Consistent with the XRD and BET results, examination by TEM revealed no readily apparent change to either the particle size or morphology for heat-treatment temperatures below the melting point of $\mathrm{LiCl}$. In contrast, as shown in Figure 5.26, heattreatment at $700^{\circ} \mathrm{C}$ resulted in significant coarsening and the formation of acicular particles. Compared to the results shown in Figure 5.11 for the $\mathrm{ZrCl}_{4}+2 \mathrm{MgO}$ system, the acicular particles show a much smaller aspect ratio. 


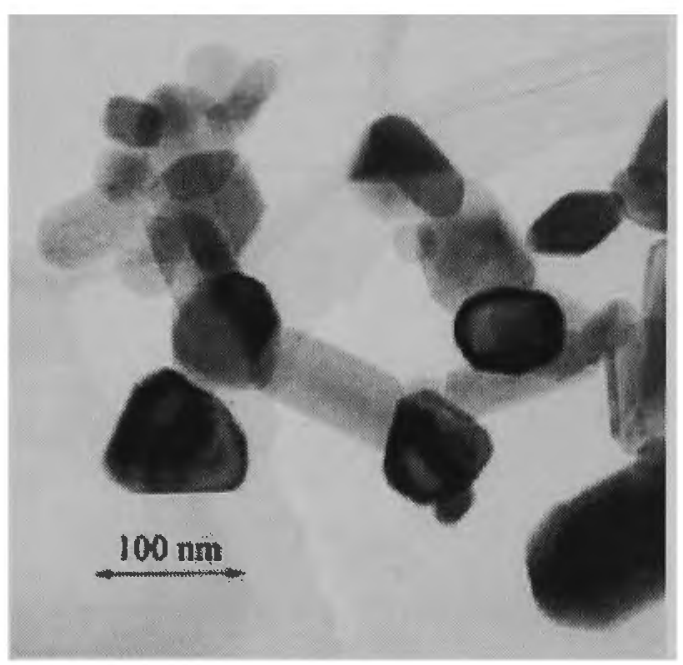

(a)

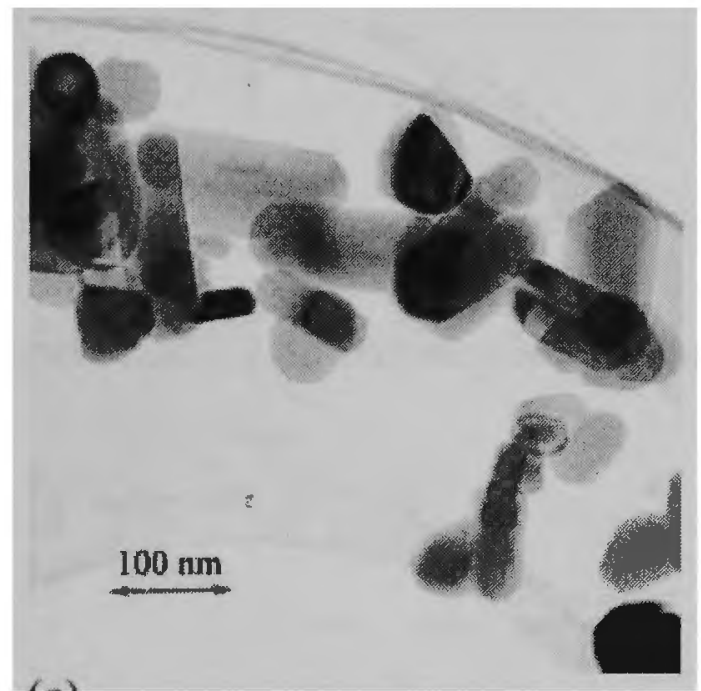

(c)

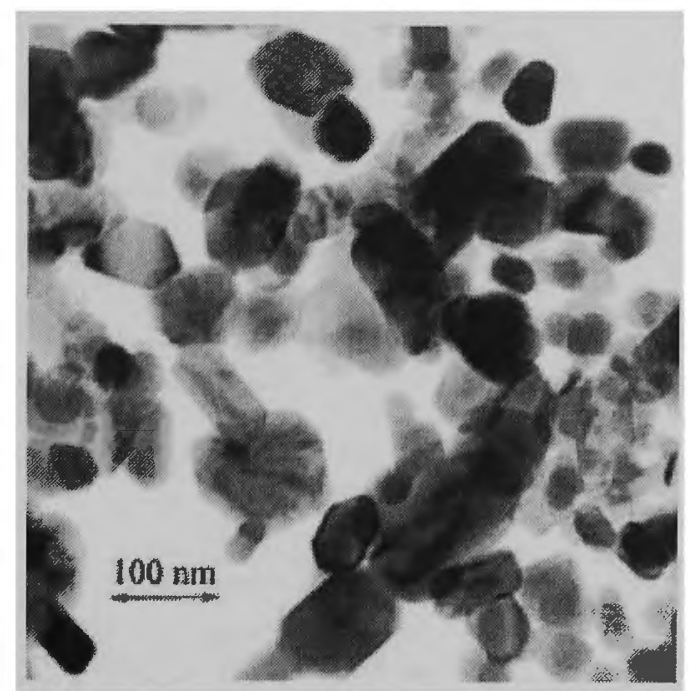

(b)

Figure 5.26 : Bright field TEM images of washed $\mathrm{ZrO}_{2}$ powders synthesised using post-reaction heat-treatment of $700^{\circ} \mathrm{C}$ for (a) 0.5 , (b) 1 , (c) 2 , and (d) 4 hours.

\section{(5.4) Lithium Hydroxide Reactions}

\section{(5.4.1) $\mathrm{ZrO}_{2}$ Synthesis}

Figure 5.27 shows the vial temperature as a function of time during milling of $\mathrm{ZrCl}_{4}+$ $4 \mathrm{LiOH}$. The temperature gradually increased for the first minute of milling as a result of heat generated by collisions of the grinding media. This was immediately followed by a sharp temperature spike, which is characteristic of the sudden heat release associated with mechanically activated combustion reactions. Following the combustion event, the vial temperature leveled off to a constant steady-state value. 


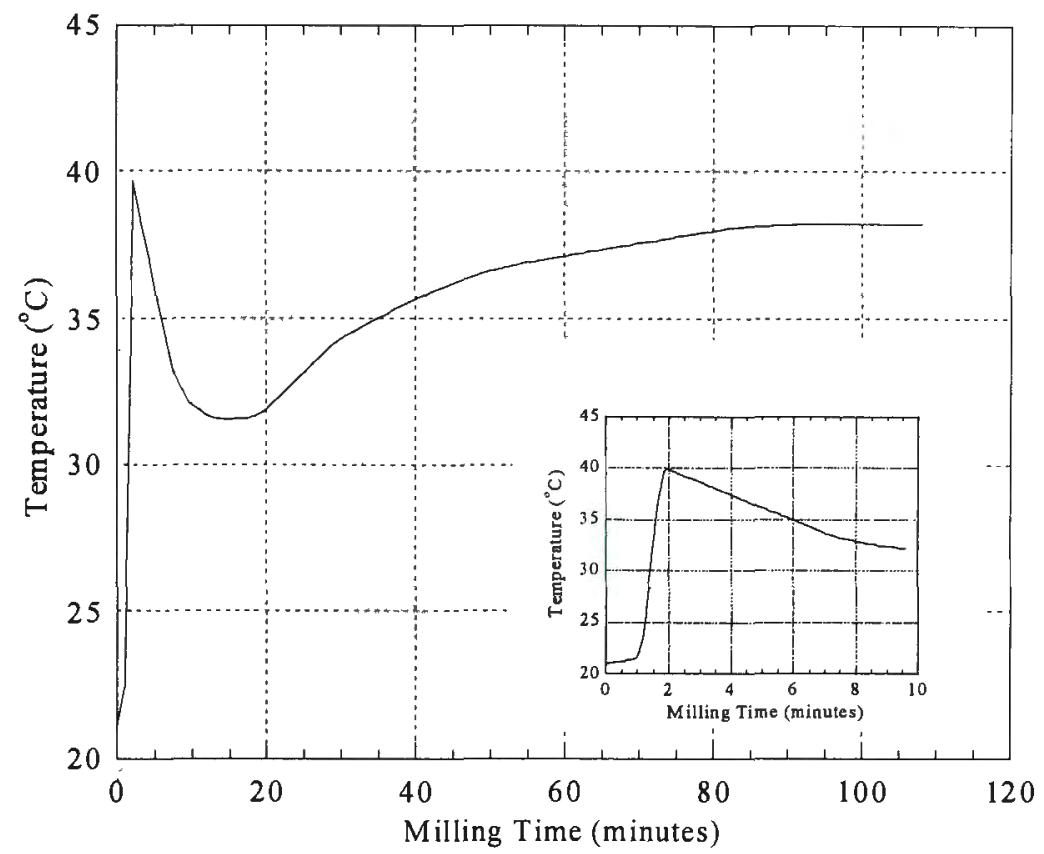

Figure 5.27 : Vial temperature as a function of time during milling of $\mathrm{ZrCl}_{4}+4 \mathrm{LiOH}$.

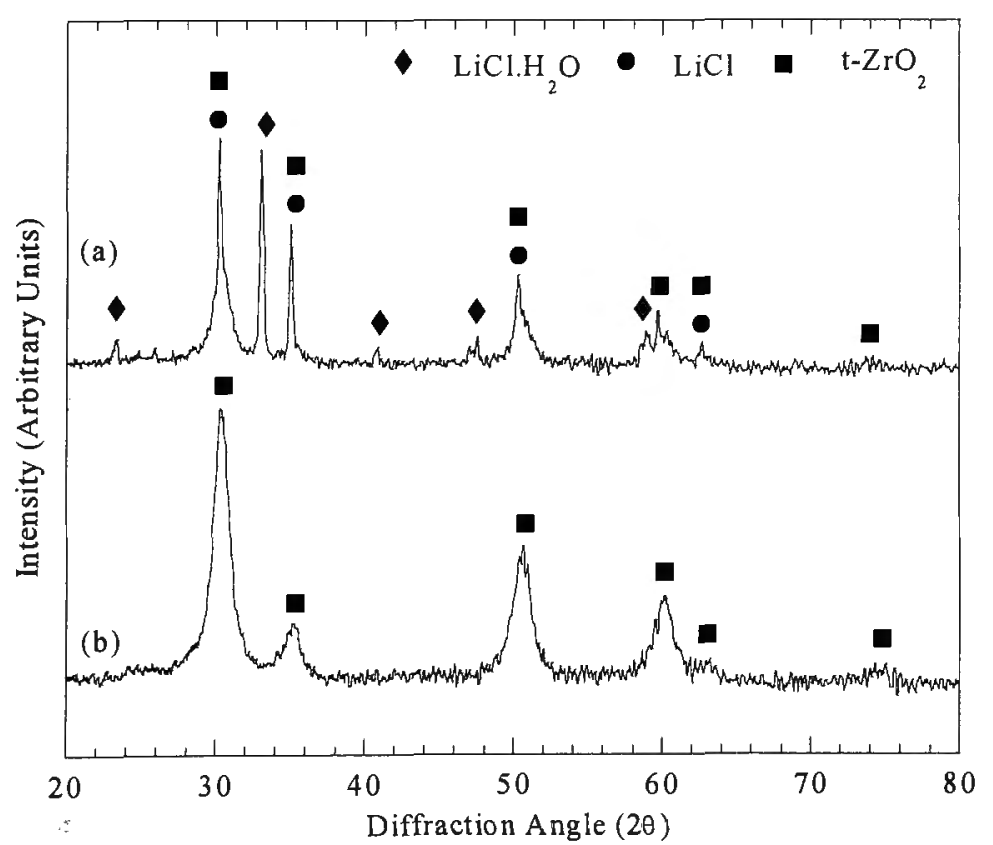

Figure 5.28 : XRD pattern of $\mathrm{ZrCl}_{4}+4 \mathrm{LiOH}$ following (a) milling for 1 minute and (b) washing. 
Immediately following the temperature spike, the powder charge was partially fused onto the grinding media and the interior of the vial. This suggests that the heat liberated by the combustion reaction was sufficient to cause at least partial melting of the reaction products. Further milling dislodged the fused powder from the grinding surfaces, thus allowing further mechanochemical processing of the powder after the combustion event.

Figure 5.28 shows the $\mathrm{XRD}$ pattern of the $\mathrm{ZrCl}_{4}+4 \mathrm{LiOH}$ reactant mixture following (a) the combustion event and (b) subsequent washing. The pattern of the combusted powder consists of diffraction peaks corresponding $\mathrm{LiCl} . \mathrm{H}_{2} \mathrm{O}, \mathrm{LiCl}$, and tetragonal $\mathrm{ZrO}_{2}$. No peaks corresponding to either reactant phase are evident, which suggests that complete reaction of the precursors occurred during the combustion event. Following washing, only those peaks corresponding to tetragonal $\mathrm{ZrO}_{2}$ remained, indicating the successful removal of the $\mathrm{LiCl}$ by-product phases.

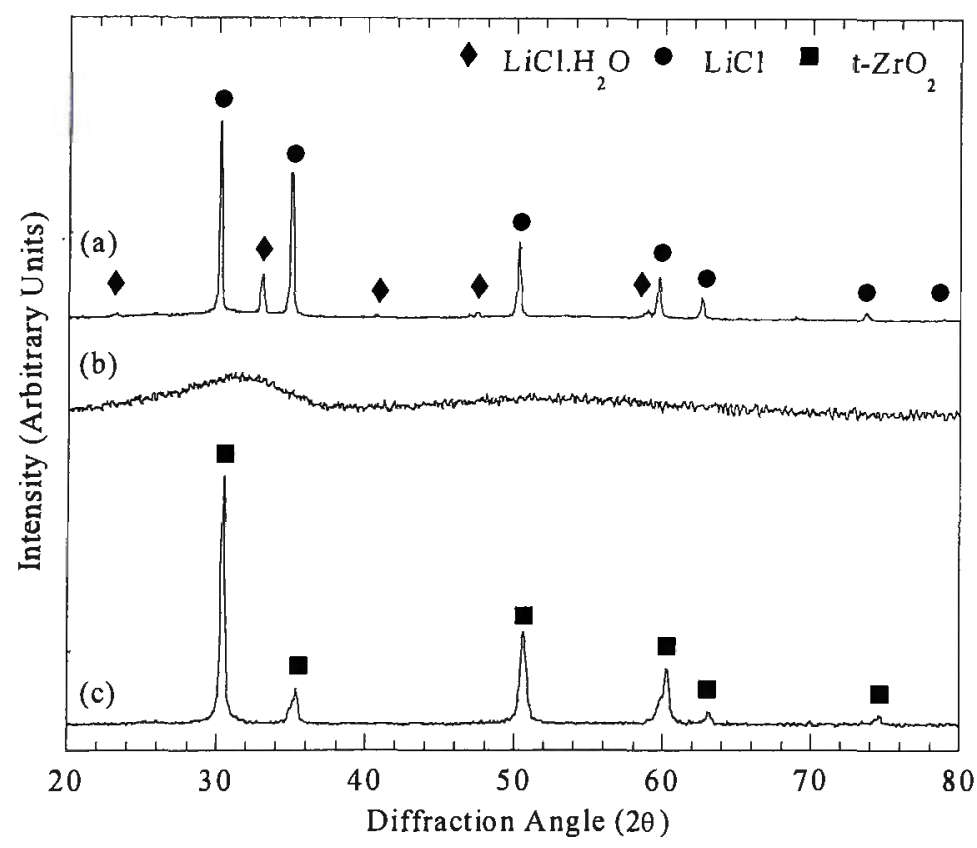

Figure 5.29 : $\mathrm{XRD}$ pattern of $\mathrm{ZrCl}_{4}+4 \mathrm{LiOH}+4 \mathrm{LiCl}$ following (a) milling for 6 hours, (b) washing, and (c) calcination at $600^{\circ} \mathrm{C}$ for 1 hour.

Dilution of the $\mathrm{ZrCl}_{4}+4 \mathrm{LiOH}$ with 4 moles of $\mathrm{LiCl}$ was found to suppress combustive reaction of the precursors. Measurement of the vial temperature during milling $\mathrm{ZrCl}_{4}+$ $4 \mathrm{LiOH}+4 \mathrm{LiCl}$ revealed only a gradual increase up to a steady-state value. The reaction 
was essentially complete following 6 hours of milling and yielded a hydrated $\mathrm{ZrO}_{2}$ product with an amorphous structure.

Illustrating the phase evolution of the diluted system during processing, Figure 5.29 shows the XRD pattern of $\mathrm{ZrCl}_{4}+4 \mathrm{LiOH}+4 \mathrm{LiCl}$ following (a) milling for 6 hours, (b) washing, and (c) calcination at $600^{\circ} \mathrm{C}$ for 1 hour. The pattern of the as-milled powder contains diffraction peaks corresponding to $\mathrm{LiCl}$ and $\mathrm{LiCl} \cdot \mathrm{H}_{2} \mathrm{O}$. No other peaks are evident, which suggests that the reaction was complete and that the $\mathrm{ZrO}_{2}$ reaction product was amorphous. Consistent with this assertion, the pattern of the washed powder contains no readily discernible diffraction peaks. Calcination of the washed powder resulted in the appearance of tetragonal $\mathrm{ZrO}_{2}$ diffraction peaks.

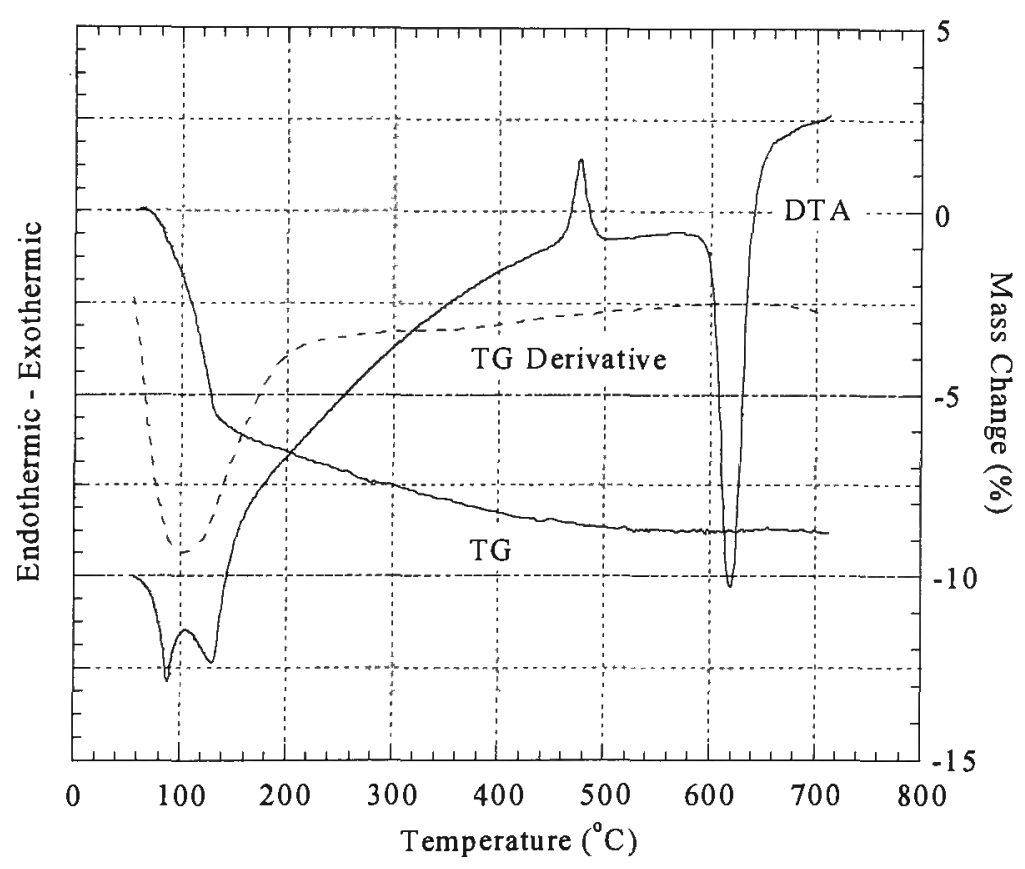

Figure 5.30 : TG-DTA traces of $\mathrm{ZrCl}_{4}+4 \mathrm{LiOH}+4 \mathrm{LiCl}$ that was milled for 6 hours.

Figure 5.30 shows TG-DTA traces of $\mathrm{ZrCl}_{4}+4 \mathrm{LiOH}+4 \mathrm{LiCl}$ that was milled for 6 hours. Mass loss due to the evolution of water vapor occurred in two stages. The first stage was comparatively rapid and appears to be associated with the two endothermic events located at approximately 86 and $130^{\circ} \mathrm{C}$. This was followed by a second stage of gradual mass loss that continued until approximately $520^{\circ} \mathrm{C}$. The exothermic peak at approximately $480^{\circ} \mathrm{C}$ corresponds to crystallization of the tetragonal $\mathrm{ZrO}_{2}$ phase. The large endothermic peak at high temperature was due to melting of the $\mathrm{LiCl}$ phase. 
The first stage of mass loss and the accompanying endothermic events can be attributed to evaporation of free water and decomposition of the $\mathrm{LiCl} . \mathrm{H}_{2} \mathrm{O}$ phase. The free water either being produced by mechanochemical reaction during milling or acquired during transfer and handling of the hygroscopic powder outside of the argon filled glovebox. The second stage of mass loss appears to be due to calcination of the amorphous $\mathrm{ZrO}_{2}$ phase. The theoretical mass percent of water produced by reaction of $\mathrm{ZrCl}_{4}+4 \mathrm{LiOH}+$ $4 \mathrm{LiCl}$ is approximately $7.23 \%$, which is slightly less than the total mass loss observed by thermal analysis of the as-milled powder. This suggests that the powder did indeed acquire some additional water from the atmosphere.

\section{(5.4.2) Synthesis of Stabilised Zirconia}

Reaction systems for the synthesis of $\mathrm{MgO}\left(\mathrm{MgCl}_{2}+2 \mathrm{LiOH}\right)$ and $\mathrm{Y}_{2} \mathrm{O}_{3}\left(2 \mathrm{YCl}_{3}+\right.$ $3 \mathrm{LiOH})$ were investigated with the objective of combining them with the basic $\mathrm{ZrO}_{2}$ synthesis reaction for manufacturing Mg-PSZ and Y-TZP powders. Both systems were found to react in a gradual manner during milling and resulted in the formation of hydrated reaction products that could be calcined to form the desired oxide phases.

Figure 5.31 shows the $\mathrm{XRD}$ pattern of $\mathrm{MgCl}_{2}+2 \mathrm{LiOH}$ following (a) milling for 6 hours, (b) washing with methanol, and (c) calcination at $400^{\circ} \mathrm{C}$ for 1 hour. The pattern of the milled powder consists of broadened diffraction peaks corresponding to anhydrous $\mathrm{LiCl}$ and $\mathrm{Mg}(\mathrm{OH})_{2}$. The pattern of the washed powder consists only of those peaks corresponding to $\mathrm{Mg}(\mathrm{OH})_{2}$, indicating the successful removal of the $\mathrm{LiCl}$ reaction by-product. Following calcination, the pattern consists only of diffraction peaks corresponding to $\mathrm{MgO}$.

Quantitative analysis using the Scherrer equation gave an average crystallite size of 5.4 $\mathrm{nm}$ for the washed $\mathrm{Mg}(\mathrm{OH})_{2}$ phase and $30.8 \mathrm{~nm}$ for the calcined $\mathrm{MgO}$. These values are in approximate agreement with estimates of the average particle diameter derived from measurements of the specific surface area. For the washed $\mathrm{Mg}(\mathrm{OH})_{2}$, the specific surface area was $224 \mathrm{~m}^{2} / \mathrm{g}$ which corresponds to an average diameter of $11.3 \mathrm{~nm}$. The specific surface area of the calcined $\mathrm{MgO}$ was $44 \mathrm{~m}^{2} / \mathrm{g}$, which corresponds to an average diameter of $38 \mathrm{~nm}$. 


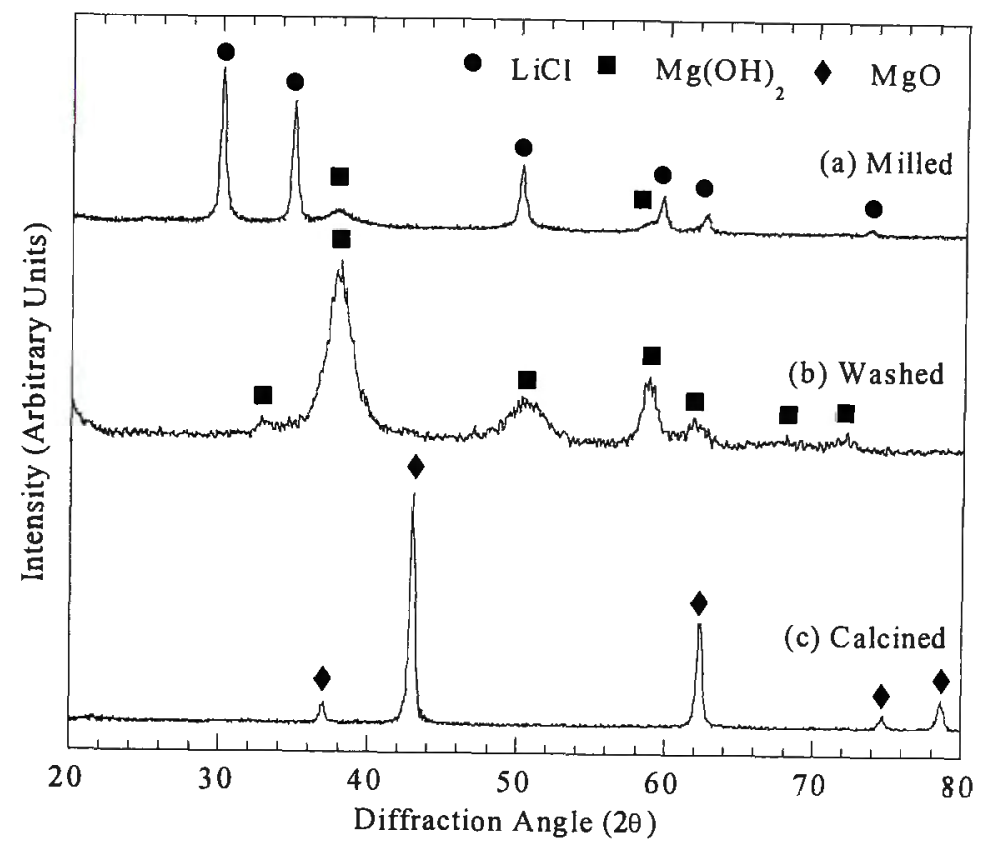

Figure 5.31 : $\mathrm{XRD}$ pattern of $\mathrm{MgCl}_{2}+2 \mathrm{LiOH}$ following (a) milling for 6 hours, (b) methanol washing, and (c) calcination at $400^{\circ} \mathrm{C}$ for 1 hour.

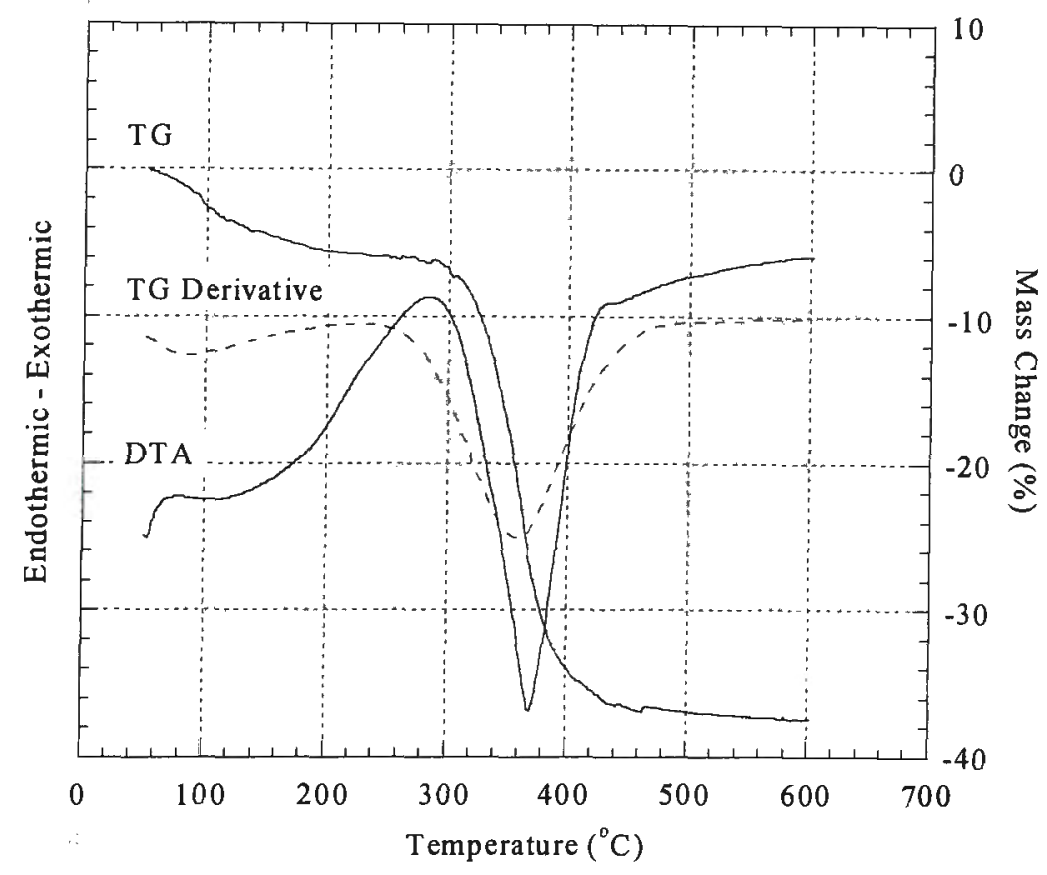

Figure 5.32 : TG-DTA traces of $\mathrm{MgCl}_{2}+2 \mathrm{LiOH}$ that was milled for 6 hours and washed. 
Figure 5.32 shows the results of simultaneous TG-DTA of the washed $\mathrm{Mg}(\mathrm{OH})_{2}$ powder. The DTA curve shows a broad endothermic peak centered around $365^{\circ} \mathrm{C}$. Correlated with this endothermic peak is a mass loss of $\sim 31 \%$, which is in good agreement with theoretically predicted mass loss of $30.9 \%$ associated with calcination of $\mathrm{Mg}(\mathrm{OH})_{2}$ to $\mathrm{MgO}$. The TG curve also shows an initial mass loss of $\sim 6 \%$ which began with the heating ramp and continued until $\sim 280^{\circ} \mathrm{C}$. This can be attributed to the loss of adsorbed methanol molecules, which were acquired during washing.

Figure 5.33 shows the XRD pattern of $\mathrm{YCl}_{3}+3 \mathrm{LiOH}$ following (a) milling for 6 hours, (b) washing, and (c) calcination at $400^{\circ} \mathrm{C}$ for 1 hour. The pattern of the milled powder consists solely of broadened diffraction peaks corresponding to anhydrous $\mathrm{LiCl}$. No peaks corresponding to any other phases are apparent, which suggests that the $\mathrm{Y}(\mathrm{OH})_{3}$ reaction product was either amorphous or at least poorly crystalline. The pattern of the washed powder contains no readily evident diffraction peaks, indicating the successful removal of the $\mathrm{LiCl}$ reaction by-product. Following calcination, the pattern consists of diffraction peaks corresponding to $\mathrm{Y}_{2} \mathrm{O}_{3}$.

Figure 5.34 shows TG-DTA traces of $\mathrm{YCl}_{3}+3 \mathrm{LiOH}$ that was milled for 6 hours and washed. The only clearly identifiable thermal event detected in the DTA trace is endothermic event at approximately $120^{\circ} \mathrm{C}$, which can most likely be attributed to the loss of physiosorbed solvent molecules. No exothermic event corresponding to crystallisation is evident, indicating that the $\mathrm{Y}(\mathrm{OH})_{3}$ product phase was simply disordered rather than amorphous. The TG derivative curve shows three maxima in the rate of mass loss, presumably corresponding to the progressive decomposition of the $\mathrm{Y}(\mathrm{OH})_{3}$ to $\mathrm{Y}_{2} \mathrm{O}_{3}$.

Reactant mixtures for the synthesis of stabilised zirconia were found to exhibit essentially identical chemical and structural evolution during processing as that shown by the basic $\mathrm{ZrO}_{2}$ system. In the absence of any diluent, reaction of the precursors occurred by mechanically activated combustion and resulted in the formation of the crystalline powders. Diluted reactant mixtures underwent reaction in a gradual manner during milling and formed hydrated gels with an amorphous structure 


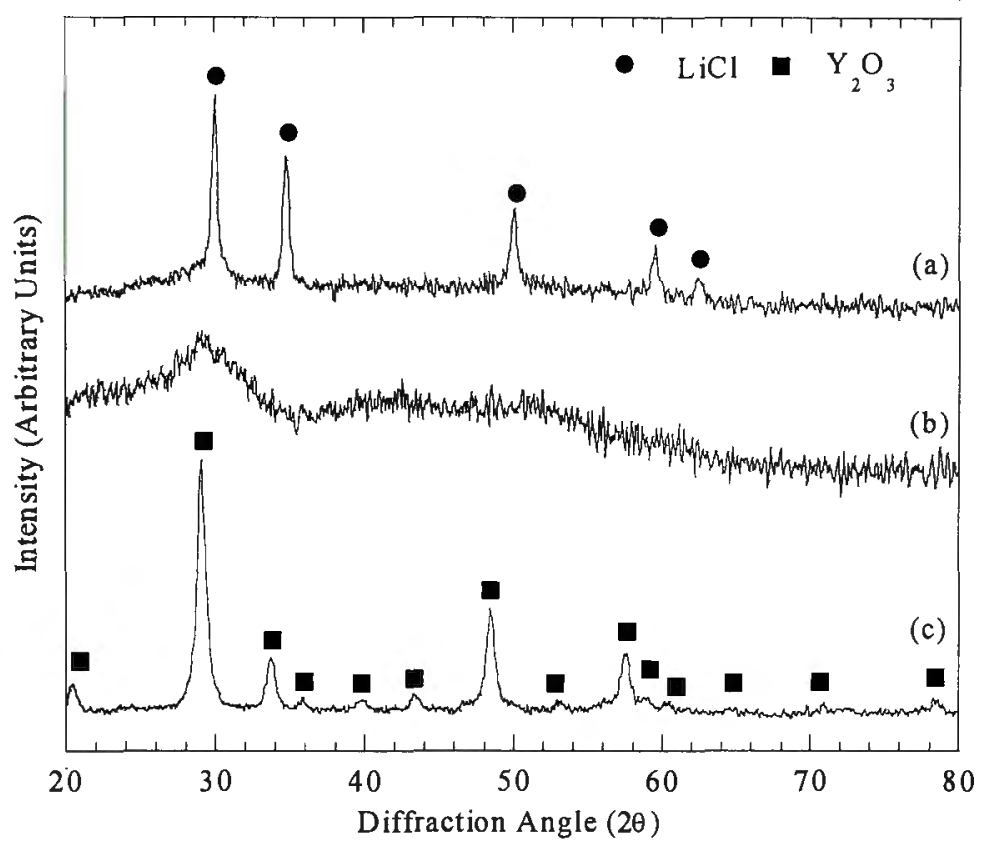

Figure 5.33 : XRD pattern of $\mathrm{YCl}_{3}+3 \mathrm{LiOH}$ following (a) milling for 6 hours, (b) washing, and (c) calcination at $400^{\circ} \mathrm{C}$ for 1 hour.

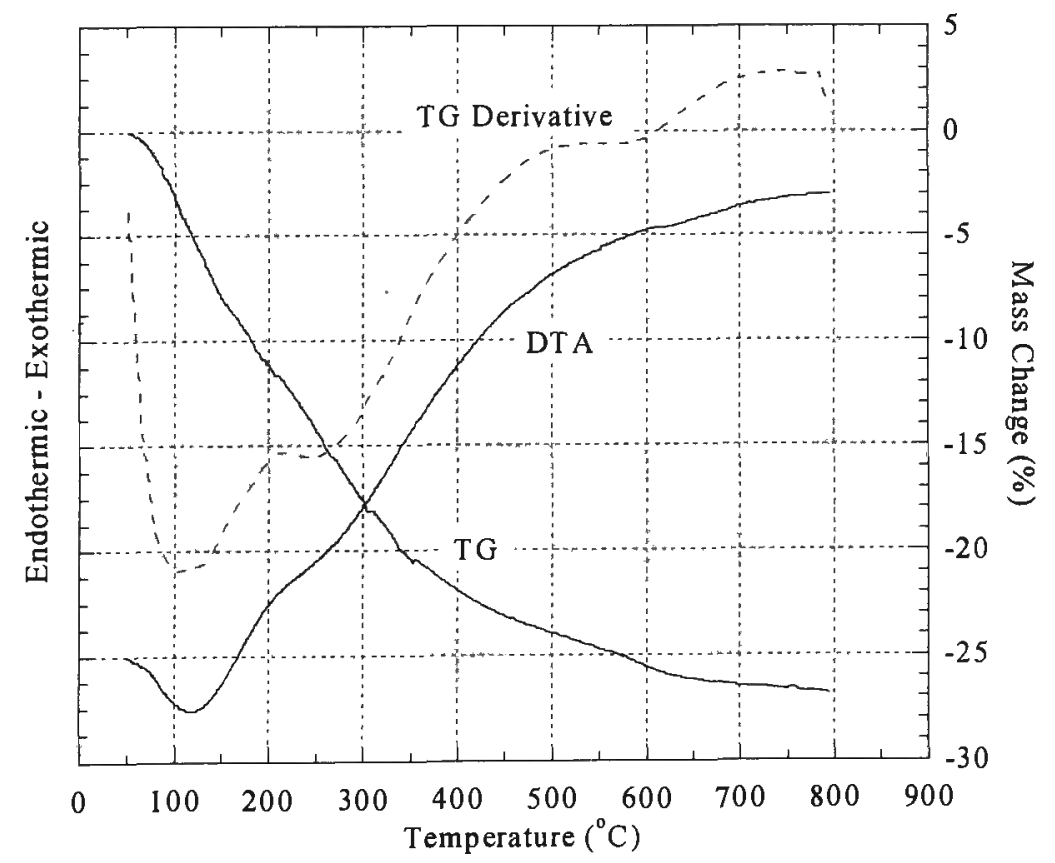

Figure 5.34 : TG-DTA traces of $\mathrm{YCl}_{3}+3 \mathrm{LiOH}$ that was milled for 6 hours and washed. 
As shown by XRD in Figure 5.35 (a), the diluted reactant mixtures were found to successfully yield stabilised powders. The (111) tetragonal diffraction peak shifted to a lower angle for the Y-TZP and to a higher angle for the Mg-PSZ. In contrast, the undiluted reactant mixtures, which reacted in a combustive manner, did not form the expected solid solutions. Analysis by XRD found no significant shift in the position of the (111) tetragonal diffraction peak.

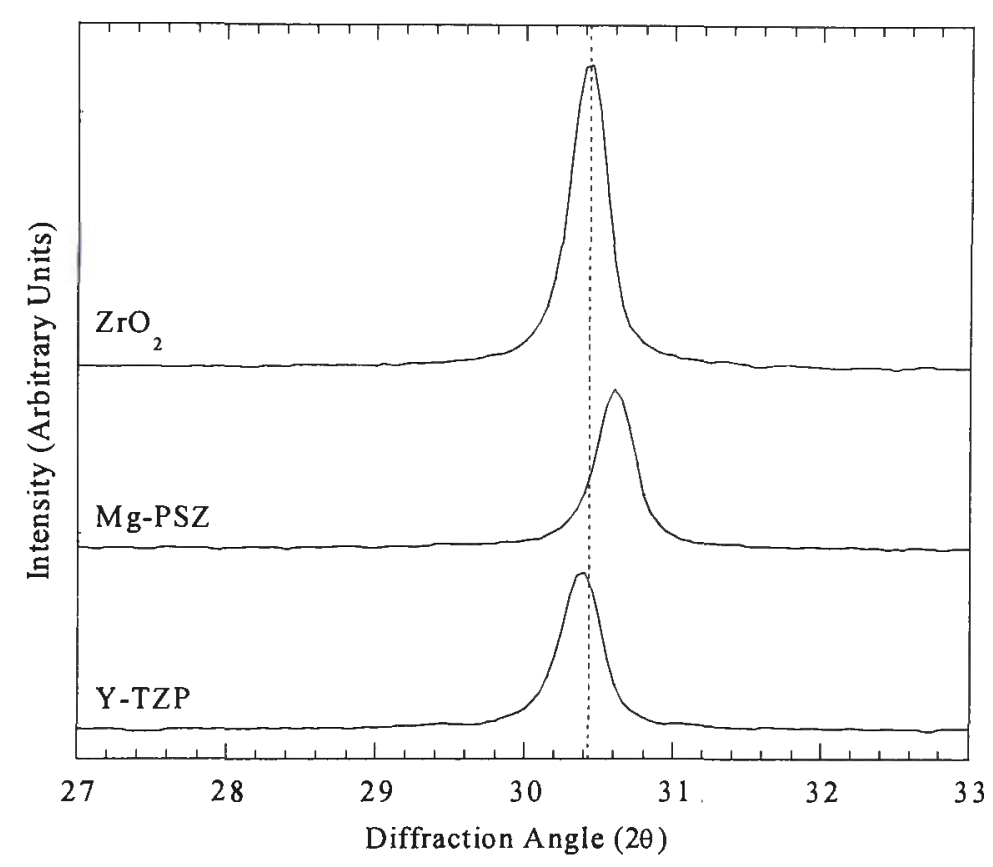

Figure 5.35 : XRD patterns of washed and calcined $\mathrm{ZrO}_{2}, \mathrm{Mg}-\mathrm{PSZ}$, and Y-TZP powders synthesised from reactant mixtures that were diluted with $4 \mathrm{LiCl}$.

\section{(5.4.3) Effect of Milling Duration}

Continued milling of the undiluted $\mathrm{ZrCl}_{4}+4 \mathrm{LiOH}$ reactant mixture after the combustion event was found to result in a progressive transformation of the tetragonal $\mathrm{ZrO}_{2}$ reaction product to the equilibrium monoclinic structure. This behaviour is illustrated in Figure 5.36, which shows XRD patterns of washed $\mathrm{ZrO}_{2}$ powders that were synthesized from $\mathrm{ZrCl}_{4}+4 \mathrm{LiOH}$ using milling times ranging from 1 minute up to 24 hours. The pattern of the powder that was prepared using a milling time of 1 minute contains only the (101) tetragonal diffraction peak. Milling for 2 hours resulted in the 
appearance of monoclinic diffraction peaks. The intensity of these peaks increased with further milling at the expense of the tetragonal peak.

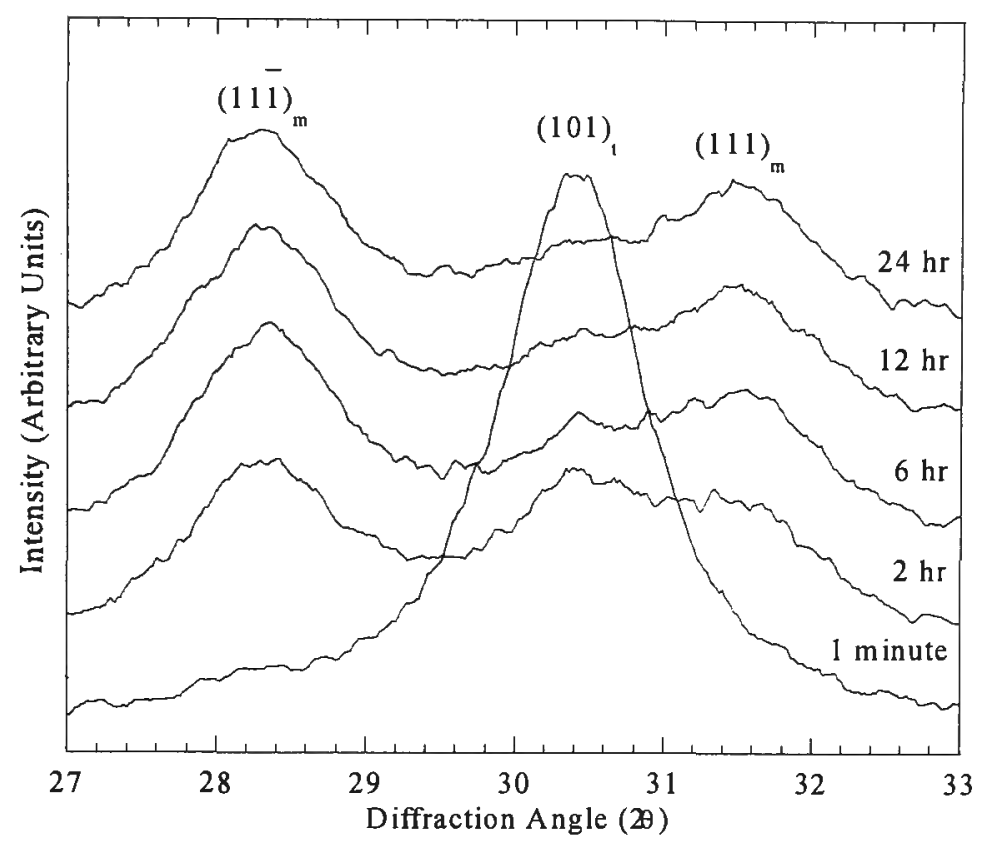

Figure 5.36 : XRD patterns of washed $\mathrm{ZrO}_{2}$ synthesised from $\mathrm{ZrCl}_{4}+4 \mathrm{LiOH}$ using milling times ranging from 1 minute up to 24 hours.

Figure 5.37 shows the phase content of the washed $\mathrm{ZrO}_{2}$ powder as a function of milling time. The monoclinic volume fraction rapidly increased with milling time up to a steady-state value of approximately $86 \%$. Also shown in Figure 5.39 is the variation in the BET particle size and XRD crystallite size. The particle size estimated from BET measurements shows a near linear increase with milling time from approximately 9 to $13 \mathrm{~nm}$. In contrast, the XRD crystallite sizes of the tetragonal and monoclinic phases remained roughly constant with milling time.

Figure 5.38 shows bright field TEM images of washed $\mathrm{ZrO}_{2}$ powders synthesized from $\mathrm{ZrCl}_{4}+4 \mathrm{LiOH}$ using milling times of (a) 1 minute and (b) 24 hours. The powder prepared using the shorter milling time consists predominantly of highly agglomerated particles. Milling for 24 hours evidently resulted in compaction of these agglomerates, which accounts for the increase in BET particle size with continued milling after the combustion event. 


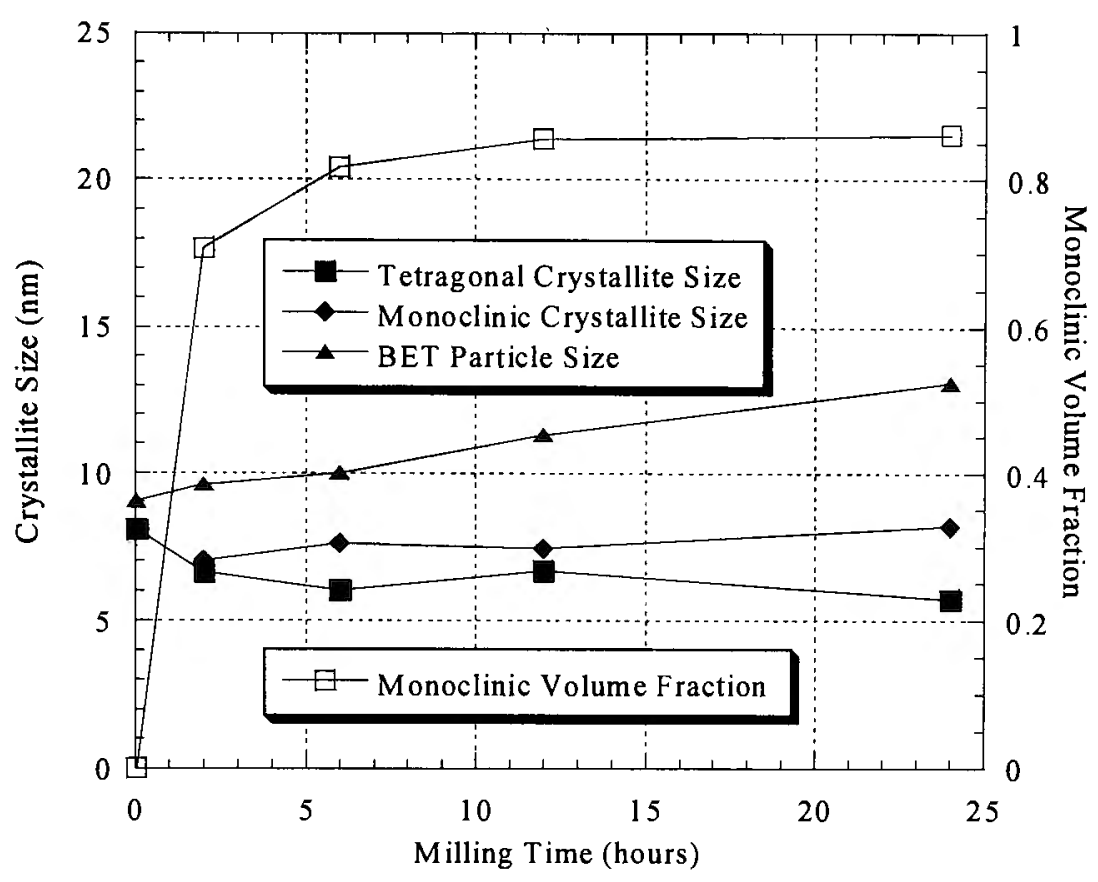

Figure 5.37 : Crystallite size and phase content as functions of milling time for $\mathrm{ZrO}_{2}$ synthesized from $\mathrm{ZrCl}_{4}+4 \mathrm{LiOH}$.

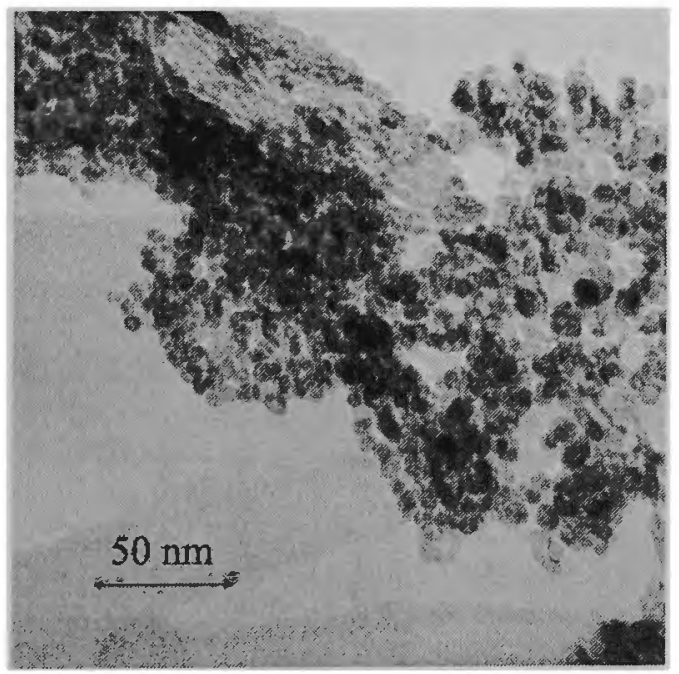

(a)

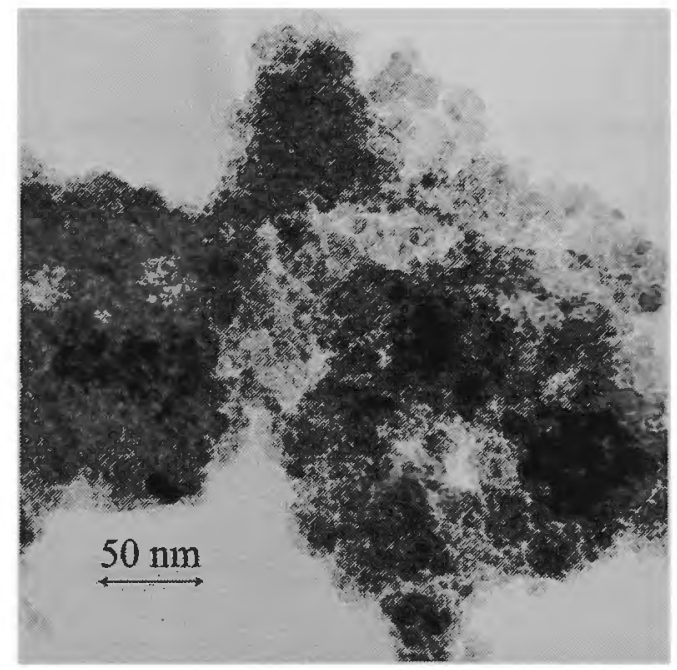

(b)

Figure 5.38 : Bright field TEM images of washed $\mathrm{ZrO}_{2}$ synthesized using milling times of (a) 1 minute and (b) 24 hours.

The undiluted reactant mixtures for the synthesis of stabilised zirconia powders were found to exhibit similar behaviour on continued milling after the combustion event. However, the rate at which the initially tetragonal structure transformed to monoclinic 
was somewhat slower, which suggests that extended milling had the effect of incorporating the stabiliser into the $\mathrm{ZrO}_{2}$.

The gradual reaction mechanism of the diluted system suggests that milling the precursors for a short duration and then driving the reaction to completion by low temperature heat-treatment could successfully be used to synthesise nanocrystalline $\mathrm{ZrO}_{2}$ powders. This possibility was confirmed by milling $\mathrm{ZrCl}_{4}+4 \mathrm{LiOH}+4 \mathrm{LiCl}$ reactant mixtures for times ranging from 10 minutes up to 6 hours and then heat-treating at $450^{\circ} \mathrm{C}$ for 30 minutes under a flowing argon atmosphere.

As previously noted, mechanochemical reaction of the diluted $\mathrm{ZrCl}_{4}+4 \mathrm{LiOH}+4 \mathrm{LiCl}$ reactant mixture resulted in the formation of a hydrated $\mathrm{ZrO}_{2}$ gel with an amorphous structure. This allows the extent of reaction with milling time to be gauged by the magnitude of the crystallisation exotherm in a DTA trace. Figure 5.39 shows DTA traces of reactant mixtures that were milled for times ranging from 10 minutes up to 6 hours. From this figure it can be seen that reaction is virtually complete following 3 hours of milling. It is also notable that increasing the milling time from 1 hour to 4 hours raised the peak temperature of the crystallisation exotherm by almost $10^{\circ} \mathrm{C}$.

Figure 5.40 shows the XRD patterns of the washed $\mathrm{ZrO}_{2}$ powders synthesised from $\mathrm{ZrCl}_{4}+4 \mathrm{LiOH}+4 \mathrm{LiCl}$ reactant mixtures. Short milling times yielded powders composed of both monoclinic and tetragonal polymorphs. The volume fraction of the monoclinic polymorph decreased with milling time. The peak broadening initially increased with milling time before decreasing to a steady state value. 


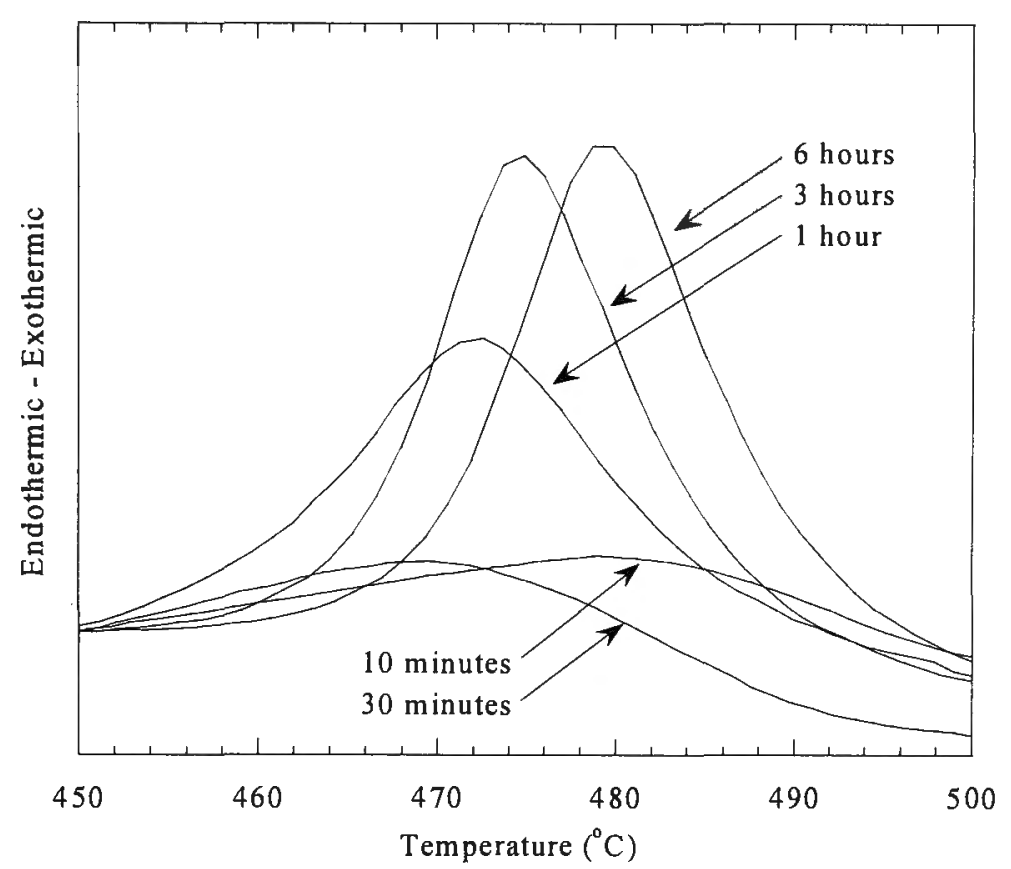

Figure 5.39 : Expanded view of the crystallisation exotherms of $\mathrm{ZrCl}_{4}+4 \mathrm{LiOH}+$ $4 \mathrm{LiCl}$ reactant mixtures that were milled for times ranging from 10 minutes to 6 hours.

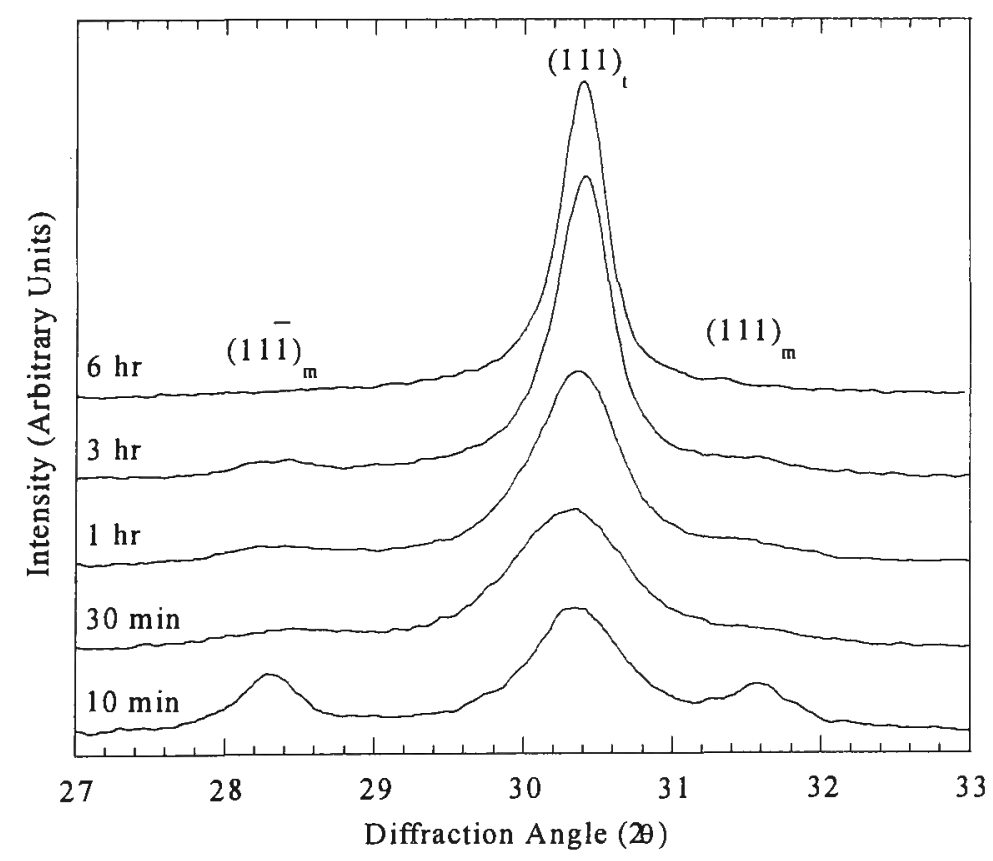

Figure 5.40 : XRD patterns of $\mathrm{ZrO}_{2}$ powders synthesised from $\mathrm{ZrCl}_{4}+4 \mathrm{LiOH}+4 \mathrm{LiCl}$ using milling times ranging from 10 minutes up to 6 hours. 
Figure 5.41 shows the crystallite size and phase content of the washed $\mathrm{ZrO}_{2}$ powder as functions of milling time. Both the XRD and BET crystallite sizes show a similar variation. The crystallite size initially decreased before reaching a minimum and then rising to a steady-state value. The tetragonal volume fraction increased from approximately $50 \%$ after 10 minutes of milling to $100 \%$ following 3 hours.

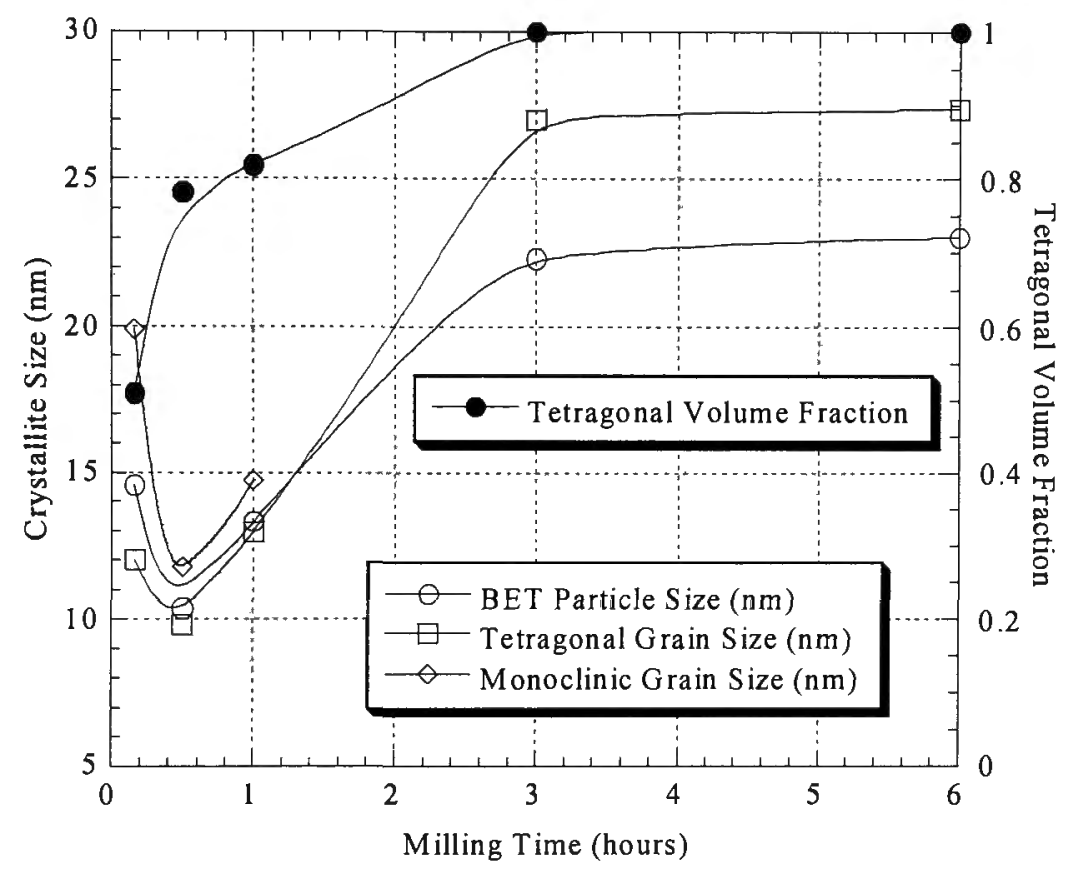

Figure 5.41 : Crystallite size and phase content as functions of milling time for washed $\mathrm{ZrO}_{2}$ synthesized from $\mathrm{ZrCl}_{4}+4 \mathrm{LiOH}+4 \mathrm{LiCl}$.

Figure 5.42 shows bright TEM images of washed $\mathrm{ZrO}_{2}$ synthesized from $\mathrm{ZrCl}_{4}+$ $4 \mathrm{LiOH}+4 \mathrm{LiCl}$ reactant mixtures using milling times of (a) 10 minutes, (b) 30 minutes, and (c) 3 hours. Powder synthesized using a milling time of 10 minutes consists of distinct crystallites. A milling time of 30 minutes yielded a powder consisting of aggregates of smaller crystallites. Following 3 hours of milling the powder consists of large particles that are composed of aggregated crystallites. 


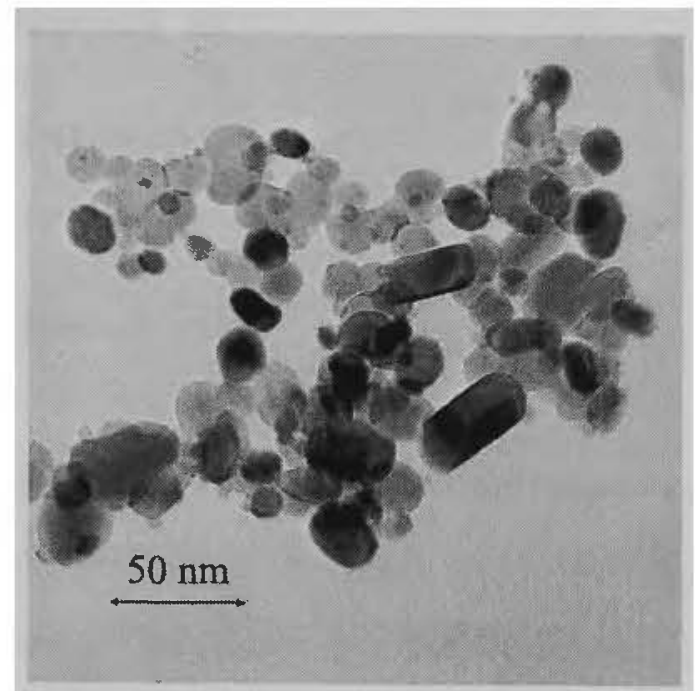

(a)

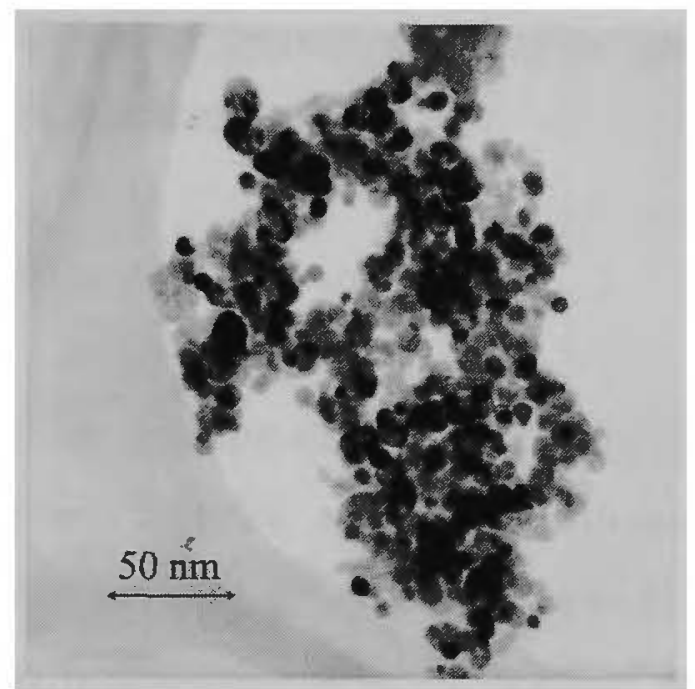

(b)

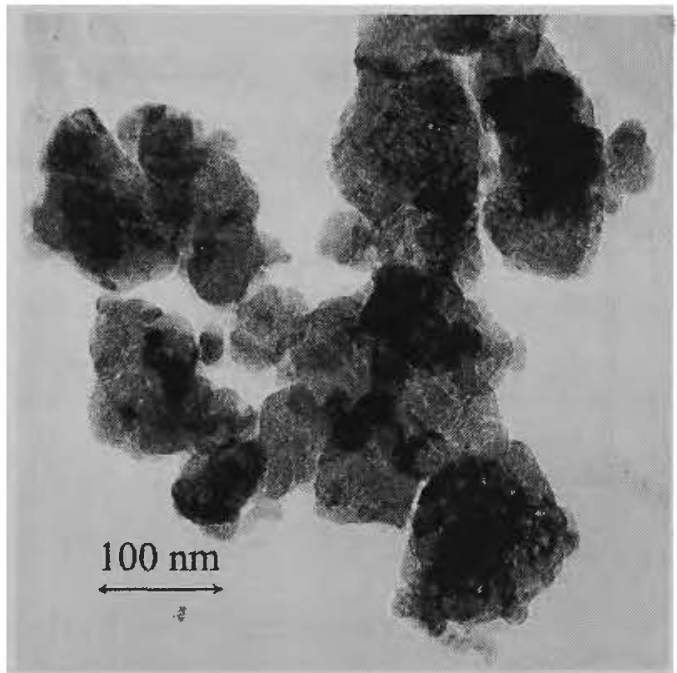

(c)

Figure 5.42 : Bright field TEM images of washed $\mathrm{ZrO}_{2}$ synthesized from $\mathrm{ZrCl}_{4}+$ $4 \mathrm{LiOH}+4 \mathrm{LiCl}$ using milling times of (a) 10 minutes, (b) 30 minutes, and (c) 3 hours. 


\section{Chapter 6}

\section{Discussion}

\section{(6.1) Introduction}

In this study, mechanically activated reaction of anhydrous chloride precursors with various exchange reagents was investigated for the synthesis of ultrafine zirconia powders. The purpose of this investigation was to identify the different reaction mechanisms, examine the effects of dilution and post-milling heat-treatment, and also to characterise the properties of the final zirconia powders, specifically the crystallite size and phase content.

\section{(6.2) Reaction Mechanisms}

All of the reaction systems that were investigated in this study involved reaction of anhydrous chloride precursors with an exchange reagent. However, despite this fundamental similarity, the three basic systems for the synthesis of $\mathrm{ZrO}_{2}$ were found to exhibit significantly different reaction kinetics. Depending on the exchange reagent used and the presence of inert diluent, reaction to form $\mathrm{ZrO}_{2}$ and by-product salt either occurred gradually during milling, by mechanically activated combustion, or only during subsequent low temperature heat treatment.

Reaction of the $\mathrm{ZrCl}_{4}+2 \mathrm{MgO}$ system was found to occur in a gradual manner during milling for all concentrations of diluent. This allowed ultrafine $\mathrm{ZrO}_{2}$ powders to be synthesised by either milling the precursors until complete reaction was achieved or by milling for a short duration and then driving the reaction to completion by low temperature heat treatment.

The kinetic behaviour of the $\mathrm{ZrCl}_{4}+4 \mathrm{LiOH}$ reaction system was found to depend on the presence of diluent. In the absence of any $\mathrm{LiCl}$ diluent, reaction of the precursors occurred in a combustive manner following approximately 1 minute of milling. Dilution of the reactant mixture with sufficient $\mathrm{LiCl}$ suppressed combustive reaction and reaction of the precursórs occurred gradually during milling. As for the $\mathrm{ZrCl}_{4}+2 \mathrm{MgO}$ reaction 
system, this allowed $\mathrm{ZrO}_{2}$ particles to be synthesised either by milling until complete reaction or by combining a short milling time with subsequent low temperature heat treatment.

The chemical evolution of the $\mathrm{ZrCl}_{4}+2 \mathrm{Li}_{2} \mathrm{O}$ reaction system during processing was found to be similar to that observed previously for mechanically activated reaction systems that used $\mathrm{CaO}$ as an exchange reagent. Milling of the reactant mixture resulted in amorphisation of the $\mathrm{ZrCl}_{4}$ reactant phase and overall microstructural refinement. Chemical reaction, with the consequent formation of ultrafine $\mathrm{ZrO}_{2}$ particles and $\mathrm{LiCl}$, only occurred during post-milling heat treatment. The mechanism of reaction during heat treatment was found to depend on the presence of $\mathrm{LiCl}$ diluent and the applied heating rate.

\section{(6.2.1) Adiabatic Reaction Temperature}

From previous studies it is known that the enthalpy change associated with formation of product phases is a key factor determining the mechanism of solid-state chemical reactions. The effect of the enthalpy change is usually expressed in terms of the adiabatic reaction temperature, which is defined as the temperature reached by the product phases as a result of the heat released by reaction. If the temperature generated by reaction is sufficient to initiate reaction in neighbouring volume elements then self propagating combustion can be induced.

For the synthesis of compounds through thermally ignited reaction of elemental precursors, it has been demonstrated empirically that reaction will generally not undergo self propagation unless the adiabatic reaction exceeds approximately $1800 \mathrm{~K}$ [94]. In contrast, for mechanochemical reduction reactions, Schaffer and McCormick [19] found that self sustaining combustion can be induced in systems with an adiabatic reaction temperature as low as $1300 \mathrm{~K}$.

In order to establish a correlation between the observed reaction mechanism and the associated enthalpy change, the adiabatic reaction temperature was calculated as a function of diluent content for each of the three basic $\mathrm{ZrO}_{2}$ synthesis systems. The 
adiabatic temperature was calculated on the assumption that all of the heat generated by reaction went towards heating the final product phases, as expressed by equation (6.1):

$$
-\Delta \mathrm{H}_{298}=\int_{298}^{\mathrm{T}_{\mathrm{ad}}} \sum \mathrm{nC}_{\mathrm{p}} \mathrm{dT}+\sum \mathrm{n} \Delta \mathrm{H}
$$

where $\Delta \mathrm{H}_{298}$ is the overall reaction enthalpy, $\mathrm{n}$ is the stoichiometry coefficient, $\mathrm{C}_{\mathrm{p}}$ is the heat capacity of the reaction product, and $\Delta \mathrm{H}$ is the enthalpy change associated with any phase change of the reaction product. The effect of the diluent content was included in the calculations by assuming that the extra salt was chemically inert and simply served as an additional sink for the heat generated by reaction.

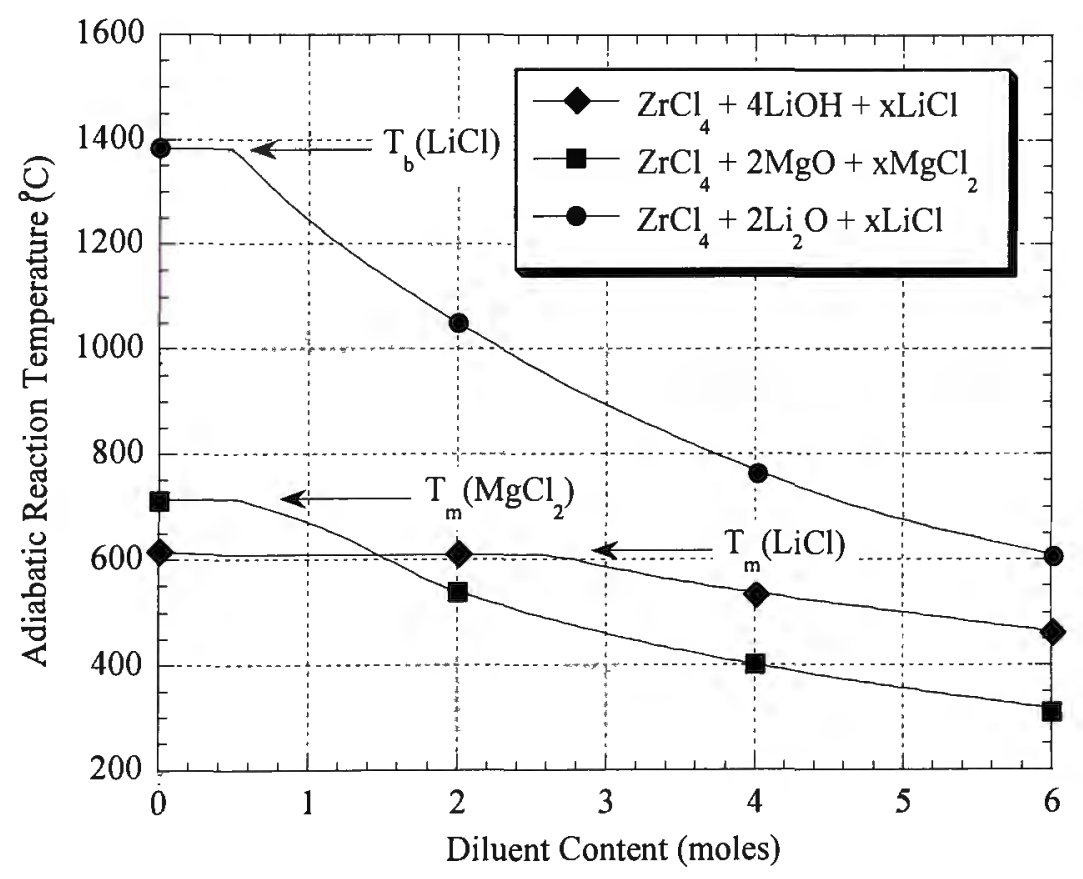

Figure 6.1 : Adiabatic reaction temperature as a function of diluent content for each of the three basic $\mathrm{ZrO}_{2}$ reaction systems.

The adiabatic temperature of the reaction system that used $\mathrm{LiOH}$ as the exchange reagent was calculated on the assumption that the reaction products were $\mathrm{ZrO}_{2}, \mathrm{LiCl}$, and $\mathrm{H}_{2} \mathrm{O}$ vapour. This is seemingly contrary to the results derived by XRD, which revealed the presence of $\mathrm{LiCl} \cdot \mathrm{H}_{2} \mathrm{O}$ in the as-milled powder. However, the temperature required for the decomposition of this phase is low $\left(\sim 98^{\circ} \mathrm{C}\right)$. As a result, it is reasonable 
to assume that $\mathrm{H}_{2} \mathrm{O}$ vapour was formed during reaction and was subsequently absorbed by the $\mathrm{LiCl}$ to form the final hydrated product phase.

\section{(6.2.2) Magnesium Oxide Reaction}

To date there have been comparatively few studies regarding the synthesis of oxide powders by mechanochemical exchange reactions with oxide exchange reagents. The majority of systems that have been investigated so far have used hydroxides or carbonates as the exchange reagent. The only oxide exchange reagent to have been studied extensively is $\mathrm{CaO}$, which was generally found to only undergo chemical reaction with chloride precursors during post-milling heat treatment [27-29]. This is in distinct contrast to the behaviour observed in the present study for the $\mathrm{ZrCl}_{4}+2 \mathrm{MgO}$ system where reaction occurred during milling. The reason for this difference kinetic behaviour is unclear given the chemical similarities between $\mathrm{CaO}$ and $\mathrm{MgO}$.

The gradual reaction mechanism of the $\mathrm{ZrCl}_{4}+2 \mathrm{MgO}$ system is consistent with the comparatively low enthalpy change associated with formation of the $\mathrm{ZrO}_{2}$ and $\mathrm{MgCl}_{2}$ product phases. As shown in Figure 6.1, the adiabatic reaction temperature does not exceed the melting point of the $\mathrm{MgCl}_{2}$ by-product phase $\left(\sim 714^{\circ} \mathrm{C}\right)$, which is significantly lower than the minimum temperature required for self propagating reaction.

\section{(6.2.3) Lithium Oxide Reactions}

The reaction mechanism of undiluted reactant mixtures for the synthesis of zirconia $\left(\mathrm{ZrO}_{2}, \mathrm{Mg}-\mathrm{PSZ}\right.$, and $\left.\mathrm{Y}-\mathrm{TZP}\right)$ powders was found to exhibit a strong dependence on the applied heating rate. The as-milled reactant mixtures underwent multistage reaction at low heating rates whereas a rapid heating rate led to single stage combustive reaction. XRD analysis showed that reaction under conditions of slow heating resulted in the formation of $\mathrm{Li}_{2} \mathrm{ZrCl}_{6}$ as an intermediate product phase, which ultimately reacted with the remaining $\mathrm{Li}_{2} \mathrm{O}$ reactant to form the final product phases of tetragonal $\mathrm{ZrO}_{2}$ and $\mathrm{LiCl}$. 
Hector et al. [95] have previously observed similar behaviour for the solid-state metathesis reaction of $\mathrm{ZrCl}_{4}$ with $\mathrm{Li}_{3} \mathrm{~N}$. Thermal analysis using a heating rate of $10^{\circ} \mathrm{C} /$ minute showed that formation of $\mathrm{ZrN}$ and $\mathrm{LiCl}$ occurred via a series of exothermic events over the temperature range of 200 to $500^{\circ} \mathrm{C}$. In contrast, measurements taken by thermocouple during rapid furnace heating showed a single exothermic event. Whether the formation of $\mathrm{Li}_{2} \mathrm{ZrCl}_{6}$ as an intermediate product phase was responsible for the multistage reaction of $\mathrm{ZrCl}_{4}$ with $\mathrm{Li}_{3} \mathrm{~N}$ during slow heating is unclear as no analysis of the intermediate product phases was reported. However, given that $\mathrm{ZrCl}_{4}$ is an initial reactant and that $\mathrm{LiCl}$ is a product phase, it would appear to at least be possible.

The formation of an intermediate chloride phases has also been observed by Ding et al. [28] in their study of mechanically activated reaction of $2 \mathrm{AlCl}_{3}+3 \mathrm{CaO}$. Heat treatment of the as-milled powder at $300^{\circ} \mathrm{C}$ resulted in formation of the mixed chloride phase $\mathrm{AlCaCl}_{6}$. Completion of the reaction, with the consequent formation of the final $\gamma-\mathrm{Al}_{2} \mathrm{O}_{3}$ and $\mathrm{CaCl}_{2}$ product phases, only occurred at heat treatment temperatures above $350^{\circ} \mathrm{C}$.

Combustive reaction of the $\mathrm{ZrCl}_{4}+2 \mathrm{Li}_{2} \mathrm{O}$ reactant mixture that had been milled for 6 hours was found to initiate at approximately $160^{\circ} \mathrm{C}$. This initiation temperature is substantially lower than those observed for similar solid-state metathesis reactions. For example, Hector and Parkin [96] found that reaction of $\mathrm{HfCl}_{4}+2 \mathrm{Li}_{2} \mathrm{O}$ initiated at a temperature above $310^{\circ} \mathrm{C}$. Similarly, for the $\mathrm{ZrCl}_{4}+2 \mathrm{Na}_{2} \mathrm{O}$ system, Gillan and Kaner [97] found an initiation temperature of approximately $300^{\circ} \mathrm{C}$.

Experimental studies of solid-state metathesis have provided supporting evidence for the idea that reaction initiates at temperatures near the point where one of the precursors decomposes or undergoes a phase transformation [39]. In the case of reactions involving $\mathrm{ZrCl}_{4}$, one could therefore reasonably expect that reaction would initiate at temperatures around the sublimation point (approximately $320^{\circ} \mathrm{C}$ ).

The significantly lower initiation temperature observed in the present case is obviously attributable to the use of high-energy mechanical processing rather than simple mixing and grinding, as was used in the solid-state metathesis reaction systems. The severe microstructural refinement and accumulation of lattice defects would have had the effect of increasing the diffusivity of the reactant species at low temperatures. As a 
consequence, combustive reaction was able to initiate at a temperature significantly lower than the sublimation point of the $\mathrm{ZrCl}_{4}$ reactant.

The addition of $\mathrm{LiCl}$ diluent to the $\mathrm{ZrCl}_{4}+2 \mathrm{Li}_{2} \mathrm{O}$ reaction system was found to significantly increase the temperature required for initiation of chemical reaction. This behaviour is consistent with increased spatial separation of the reactant phases by the $\mathrm{LiCl}$ diluent. As a consequence of this increased separation, higher temperatures would have been required to allow the interdiffusion of reactant species that is necessary for the initiation of chemical reaction.

The equilibrium phase diagram for the $\mathrm{ZrCl}_{4}-\mathrm{LiCl}$ system derived by Song et al. [98] shows a eutectic transformation at $367^{\circ} \mathrm{C}$ for a composition of $64.5 \mathrm{~mol} . \% \mathrm{ZrCl}_{4}$. Reaction of the diluted $\mathrm{ZrCl}_{4}+2 \mathrm{Li}_{2} \mathrm{O}+\mathrm{xLiCl}$ (where $\mathrm{x}=2,4$, and 6) systems could therefore reasonably be expected to have initiated at this temperature. Partial melting of the reactant mixtures would have substantially increased the rate at which the reactant species are able to interdiffuse, thus allowing reaction to occur. Thermal analysis of these reactant mixtures showed that reaction did indeed appear to initiate at temperatures close to the $\mathrm{ZrCl}_{4}-\mathrm{LiCl}$ eutectic transformation temperature.

\section{(6.2.4) Lithium Hydroxide Reactions}

As shown in Figure 6.1, the undiluted $\mathrm{ZrCl}_{4}+4 \mathrm{LiOH}$ reactant mixture is characterised by a comparatively low adiabatic reaction temperature of approximately $616^{\circ} \mathrm{C}$, which is significantly lower than the temperatures typically associated with mechanochemical combustion reactions. Nevertheless, the undiluted $\mathrm{ZrCl}_{4}+4 \mathrm{LiOH}$ reactant mixture underwent combustive reaction following approximately 1 minute of milling.

The occurrence of combustive reaction despite the low adiabatic reaction is likely due to the use of a hydrated exchange reagent. Chemical reaction of the precursors during collisions of the grinding media would have led to the evolution of $\mathrm{H}_{2} \mathrm{O}_{(\mathrm{g})}$, which could reasonably be expected to facilitate the propagation of chemical reaction to neighbouring volume elements of the powder charge either through the formation of reactive intermediates or by accelerating the interdiffusion of reactive species. 
In contrast to the $\mathrm{ZrCl}_{4}+4 \mathrm{LiOH}$ system, the reaction systems for the synthesis of $\mathrm{Y}_{2} \mathrm{O}_{3}$ $\left(\mathrm{YCl}_{3}+3 \mathrm{LiOH}\right)$ and $\mathrm{MgO}\left(\mathrm{MgCl}_{2}+2 \mathrm{LiOH}\right)$ were found to undergo reaction in a gradual manner during milling without need of dilution. This behaviour is consistent with the lower enthalpy change associated with formation of the product phases for these reaction systems.

Combustive reaction during mechanochemical processing has previously been observed in a variety of systems based on reaction of metal chlorides with alkali hydroxides. For example, Tsuzuki and McCormick [47] found that $\mathrm{CeCl}_{3}+3 \mathrm{NaOH}$ underwent mechanochemical combustion following approximately 40 seconds of milling. Similarly, Ding et al. [43] found evidence of local combustion events during milling of $1.2 \mathrm{BaCl}_{2}+12 \mathrm{FeCl}_{3}+38.4 \mathrm{NaOH}$. In both of these studies, it was shown that combustive reaction could successfully be inhibited through the addition of excess salt as an inert diluent. Similarly, it was found in the present study that the addition of $4 \mathrm{LiCl}$ to the $\mathrm{ZrCl}_{4}+4 \mathrm{LiOH}$ reactant mixture effectively suppressed combustion.

\section{(6.3) Agglomeration Behaviour}

Mechanochemical reaction of $\mathrm{ZrCl}_{4}+2 \mathrm{MgO}$ resulted in the formation of crystalline $\mathrm{ZrO}_{2}$ particles and $\mathrm{MgCl}_{2}$. However, the powder recovered by washing of the as-milled powder was a highly agglomerated $\mathrm{Zr}(\mathrm{OH})_{4}$ gel with an amorphous structure. This behaviour could plausibly be explained by a chemical interaction with the wash water consisting of partial dissolution of the $\mathrm{ZrO}_{2}$ particles followed by precipitation. Dissolution would result in the formation of a supersaturated solution containing aqueous hydroxide species that could subsequently precipitate out as an amorphous $\mathrm{Zr}(\mathrm{OH})_{4}$ gel.

The specific surface area of a spherical particle scales with the reciprocal of radius. A reduction in particle size from the micron scale to the nanometer regime would therefore increase the fraction of the particle exposed to the solvent by several orders of magnitude, thus substantially increasing the kinetics of dissolution. In addition, the tighter radius of curvature of ultrafine particles leads to a greater thermodynamic driving force for dissolution, as expressed by the Ostwald-Freundlich equation: 


$$
\mathrm{S}=\mathrm{S}_{0} \exp \left(\frac{2 \gamma_{\mathrm{sl}} \mathrm{V}}{\mathrm{RT}}\right)
$$

where $S_{0}$ is the equilibrium solubility for a flat surface (i.e. $r=\infty$ ), $V$ is the molar volume of the material, $\gamma_{\mathrm{sl}}$ is the solid-liquid interfacial energy, $\mathrm{R}$ is the gas constant, $\mathrm{T}$ is the temperature, and $\mathrm{r}$ is the particle radius. As a consequence ultrafine powder particles are expected to show a heightened tendency towards dissolution compared to conventional coarse-grained particles [99].

In addition to the thermodynamic factors arising from the fine particle size, the presence of mechanically induced crystalline disorder in the as-milled powder would also be expected to substantially increase the tendency towards dissolution. Previous studies of mineral systems have shown that mechanical milling can enhance leaching rates beyond that expected solely from the increase in specific surface area [100]. This is consistent with the observation that post-milling heat-treatment of the $\mathrm{ZrCl}_{4}+2 \mathrm{MgO}$ reactant mixture yielded a crystalline $\mathrm{ZrO}_{2}$ powder with minimal hard agglomeration. Heattreatment would have allowed recovery of the mechanically induced crystal disorder, thus decreasing the reactivity of the $\mathrm{ZrO}_{2}$ particles towards the wash solvent.

Rinsing of the washed powders with methanol prior to drying and calcination was found to have a significant effect on the agglomeration behaviour of the final powder. Methanol rinsed powders were found to be soft and were readily broken down with gentle grinding.

The reduction in hard agglomerate formation arising from alcohol rinsing has been attributed to the lower surface tension of alcohol solvents compared to water. Capillary forces arising from the surface tension of the solvent can result in significant shrinkage of a hydrated gel during drying and calcination, which would bring the particles into close contact and thereby allow the formation of hard agglomerates. However, the capillary pressures developed in nanoparticulate systems will be high regardless of the solvent used. As a result, the formation of hard agglomerates would be expected for both the alcohol and water washed powders. It would thus appear that alcohol washing must instead affect the nature of the interaction between the particles during washing and subsequent calcination. 
Figure 6.2 shows a schematic illustration of the mechanism proposed by Lee and Readey [6] as an explanation for the formation of hard agglomerates in water washed powders. During washing, water molecules form hydrogen bonded bridges between the terminal hydroxyl groups on neighbouring particles. Subsequent drying results in the loss of these hydrogen bonded water molecules, which draws the particles together and results in the formation of direct hydrogen bonds between the terminal hydroxyl groups of the neighbouring particles. Hard agglomerates are then able to form during further dehydration and calcination by a condensation type reaction.

Kaliszewski and Heur [110] found that dispersion of hydrous $\mathrm{ZrO}_{2}$ in ethanol results in the replacement of surface hydroxyls with ethoxide groups, which prevents chemical bonding between particles during subsequent drying and calcination. As a consequence, the formation of hard agglomerates is inhibited. As illustrated schematically in Figure 6.3, excess ethanol molecules are unable to form hydrogen-bonded bridges between the surface ethoxide groups on neighbouring particles. Together with steric effects, this effectively prevents the particles from entering close proximity during drying. Further drying and calcination merely results in the removal of the ethoxide groups without the formation of hard agglomerates between particles.

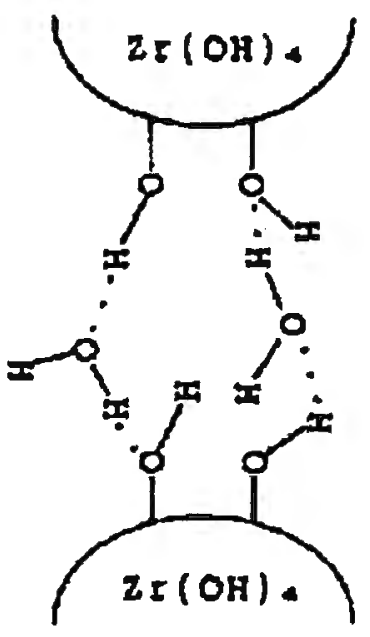

(a)

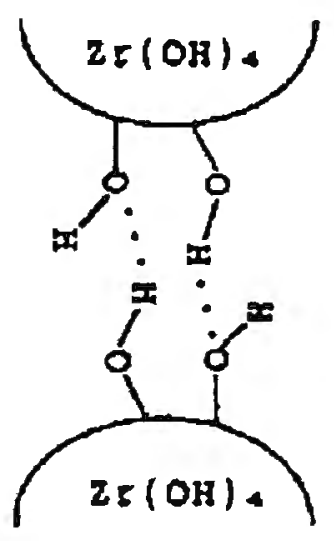

(b)

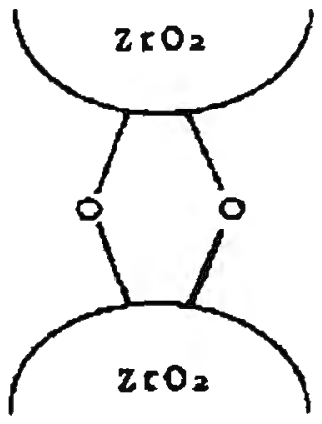

(c)

Figure 6.2 : Schematic illustration showing the mechanism of hard agglomerate formation in water washed $\mathrm{Zr}(\mathrm{OH})_{4}$ as proposed by Lee and Readey. Adapted from [6]. 

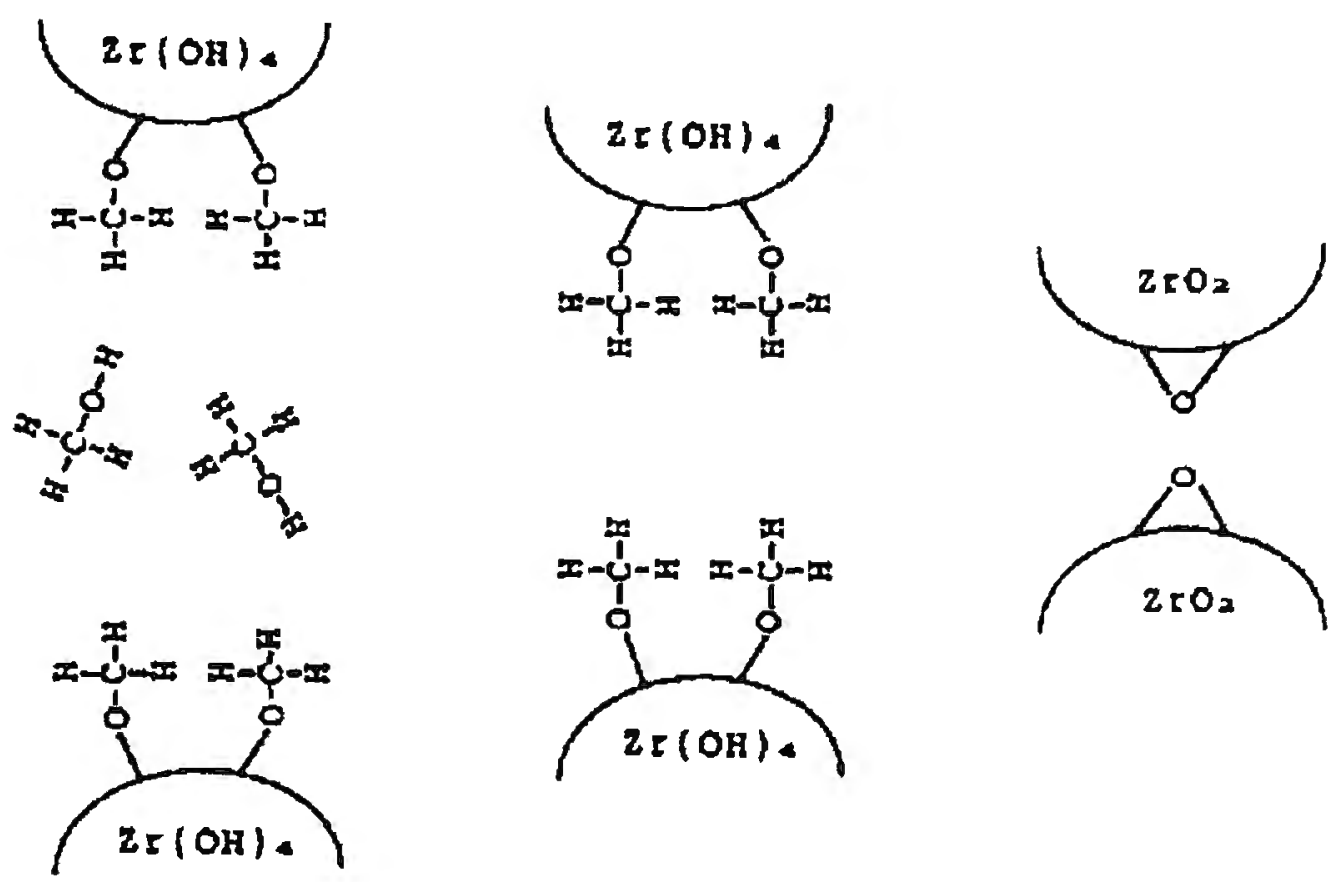

(a)

(b)

(c)

Figure 6.3 : Schematic illustration showing the inhibition of hard agglomerate formation in methanol rinsed $\operatorname{Zr}(\mathrm{OH})_{4}$ as proposed by Lee and Readey. Adapted from [6].

\section{(6.4) Particle and Crystallite Size}

\section{(6.4.1) Magnesium Oxide Reaction}

The average crystallite and particle size of the $\mathrm{ZrO}_{2}$ powder formed during milling of the $\mathrm{ZrCl}_{4}+2 \mathrm{MgO}+\mathrm{xMgCl}_{2}$ system was found to be independent of the diluent concentration. This is consistent with the view that the particle size of the reaction product is determined primarily by the effective reaction volume, which is a function of the microstructural scale of the reactants and the milling conditions. Similar results were obtained by Tsuzuki and McCormick [51] in their study of the mechanochemical synthesis of sulfide compounds. Provided that the salt volume fraction was high enough to form separable particles, there was no appreciable variation in particle size with dilution.

The behaviour of the $\mathrm{ZrCl}_{4}+2 \mathrm{MgO}$ with regard to milling time is consistent with the expectation that the microstructural scale of the reactant microstructure determines the 
crystallite size of the reaction products. Previous studies of mechanical grinding have shown that the crystallite size decreases with milling time down to a lower limit that is determined by the mechanical properties of the material.

Similar behaviour has been observed by Cukrov et al. [46] in their study of the synthesis of $\mathrm{SnO}_{2}$ by mechanochemical reaction of $\mathrm{SnCl}_{2}+\mathrm{Na}_{2} \mathrm{CO}_{3}$. Both the XRD crystallite size and BET particle size were observed to decrease with milling time until reaching a lower limit after approximately 8 hours. However, it is notable that for all milling times, the particle size determined by BET was significantly larger than the XRD crystallite size, which indicates the presence of some agglomeration. In contrast, the BET particle size and XRD crystallite size of the $\mathrm{ZrO}_{2}$ formed from $\mathrm{ZrCl}_{4}+2 \mathrm{MgO}$ in the present study were in close agreement for all milling times.

Subsequent heat treatment of the milled $\mathrm{ZrCl}_{4}+2 \mathrm{MgO}$ reactant mixture at temperatures below the melting point of the $\mathrm{MgCl}_{2}$ reaction by-product $\left(\sim 714^{\circ} \mathrm{C}\right)$ was not found to significantly alter the $\mathrm{ZrO}_{2}$ particle size. This indicates that the solid $\mathrm{MgCl}_{2}$ matrix effectively inhibited particle growth, thus allowing the crystallinity to be improved without simultaneous coarsening. In contrast, post-milling heat treatment at $750^{\circ} \mathrm{C}$ resulted in rapid particle growth, which was presumably due to the higher atomic mobility in molten salt.

Previous studies of the effect of post-milling heat treatment on the average particle size found that some particle growth did occur at temperatures below the melting point of the salt matrix. Tsuzuki and McCormick [47] found that progressively raising the calcination temperature of milled $\mathrm{CeCl}_{3}+3 \mathrm{NaOH}+12 \mathrm{NaCl}$ from 500 to $800^{\circ} \mathrm{C}$ increased the average particle size of the $\mathrm{CeO}_{2}$ product from approximately 10 to 40 nm.

\section{(6.4.2) Lithium Oxide Reactions}

A previous investigation into the synthesis of ultrafine $\mathrm{ZrO}_{2}$ powders by mechanically activated reaction of $\mathrm{ZrCl}_{4}+2 \mathrm{CaO}$ found that the particle size of the product formed during heat treatment could be directly related to the microstructural scale of the asmilled structure [29]. However, such an analysis assumes that no significant particle 
growth occurs during reaction. This assumption is likely to be invalid for reaction systems that are characterised by a large enthalpy change.

As shown in Table 6.1, the heat liberated by reaction of the $\mathrm{ZrCl}_{4}+2 \mathrm{Li}_{2} \mathrm{O}+\mathrm{xLiCl}$ system under adiabatic conditions is sufficient to raise the temperature beyond the melting point of $\mathrm{LiCl}$ for all diluent concentrations. This would allow growth of the $\mathrm{ZrO}_{2}$ product phase through interparticle sintering and diffusional mass transfer. However, the severity of this particle growth and sintering would decrease with the lower temperatures and higher salt volume fractions associated with higher levels of dilution, thus accounting for the reduction in particle and crystallite size.

Similar behaviour has been observed for solid-state metathesis reactions. Hector et al. [96] found that the addition of sufficient $\mathrm{LiCl}$ diluent to reactant mixtures consisting of metal chloride and $\mathrm{Li}_{3} \mathrm{~N}$ reduced the crystallite size of the metal nitride product from almost $100 \mathrm{~nm}$ down to approximately $10 \mathrm{~nm}$. Similarly, Wiley and Kaner [101] found that the addition of $16 \mathrm{NaCl}$ to $\mathrm{MoCl}_{5}+\mathrm{Na}_{2} \mathrm{~S}$ reduced the crystallite size of the $\mathrm{MoS}_{2}$ product phase from $45 \mathrm{~nm}$ down to $8 \mathrm{~nm}$. In both of these studies, estimates of the crystallite size were solely derived from measurements of the XRD peak broadening. As a result it is unknown whether the particle size was also reduced by dilution, as was observed in the present study through the use of BET gas adsorption and TEM. It is possible that without the severe microstructural refinement associated with high-energy milling that only the crystallite size is reduced by dilution.

\begin{tabular}{cccc}
\hline Diluent & \multicolumn{3}{c}{ Adiabatic Reaction Temperature $\left({ }^{\circ} \mathrm{C}\right)$} \\
Content $(\mathrm{x})$ & $\mathrm{ZrO}_{2}$ & Mg-PSZ & Y-TZP \\
\hline 0 & 1383 & 1383 & 1383 \\
2 & 1047 & 1003 & 1034 \\
4 & 772 & 731 & 764 \\
6 & 610 & 610 & 610 \\
\hline
\end{tabular}

Table 6.1 : Adiabatic reaction temperature as a function of diluent content for the $\mathrm{ZrO}_{2}$, Mg-PSZ, and Y-TZP synthesis systems. 
For a given quantity of diluent in the initial reactant mixture, the average particle and crystallite size of the stabilised zirconia powders was found to be smaller than that for the undoped $\mathrm{ZrO}_{2}$. As shown in Table 6.1, the reaction systems for the synthesis of $\mathrm{Mg}$ PSZ and Y-TZP are characterised by slightly lower adiabatic reaction temperatures than the $\mathrm{ZrCl}_{4}+2 \mathrm{Li}_{2} \mathrm{O}+\mathrm{xLiCl}$ system. As a result, the degree of particle growth experienced as a result of the heat released during reaction may have been lessened. Alternatively, the reduced particle size could possibly be attributed to an increase in the number of zirconia particles that were nucleated during reaction. The $\mathrm{MgO}$ or $\mathrm{Y}_{2} \mathrm{O}_{3}$ product formed by reaction of $\mathrm{MgCl}_{2}+\mathrm{Li}_{2} \mathrm{O}$ or $2 \mathrm{YCl}_{3}+3 \mathrm{Li}_{2} \mathrm{O}$ could have induced the formation of $\mathrm{ZrO}_{2}$ through equations (6.3) and (6.4):

$$
\begin{aligned}
& \mathrm{ZrCl}_{4}+2 \mathrm{MgO} \rightarrow \mathrm{ZrO}_{2}+2 \mathrm{MgCl}_{2} \\
& 3 \mathrm{ZrCl}_{4}+2 \mathrm{Y}_{2} \mathrm{O}_{3} \rightarrow 3 \mathrm{ZrO}_{2}+4 \mathrm{YCl}_{3}
\end{aligned}
$$

As a consequence, a greater number of $\mathrm{ZrO}_{2}$ particles would be nucleated during the overall reaction than otherwise would be if $\mathrm{ZrO}_{2}$ particles were only formed by reaction of $\mathrm{ZrCl}_{4}+2 \mathrm{Li}_{2} \mathrm{O}$.

\section{(6.4.3) Lithium Hydroxide Reactions}

Combustive reaction of the undiluted $\mathrm{ZrCl}_{4}+4 \mathrm{LiOH}$ was found to yield a highly agglomerated $\mathrm{ZrO}_{2}$ powder with an average crystallite size of approximately $9 \mathrm{~nm}$. Continued milling after the combustion event was found to result in a progressive increase in the particle size as estimated from the specific surface area, which was shown to be due to compaction of the particle agglomerates.

As noted previously, the $\mathrm{ZrCl}_{4}+4 \mathrm{LiOH}$ system is characterised by a comparatively low adiabatic reaction temperature of approximately $620^{\circ} \mathrm{C}$. However, this temperature is nevertheless above the sublimation point of the $\mathrm{ZrCl}_{4}$ reactant and the melting point of the $\mathrm{LiCl}$ product. The highly agglomerated state of the $\mathrm{ZrO}_{2}$ powder is therefore likely due to melting and vaporisation, which would have allowed $\mathrm{ZrO}_{2}$ particles to form without effective separation by the $\mathrm{LiCl}$ salt by-product. 
The average particle and crystallite size of the $\mathrm{ZrO}_{2}$ product formed by reaction of the diluted $\mathrm{ZrCl}_{4}+4 \mathrm{LiOH}$ reaction system was found to decrease with milling time before increasing up to a steady-state value. The initial decrease in particle and crystallite can be attributed to increased microstructural refinement of the reactant phases. As a result of this refinement, the crystallite size of the $\mathrm{ZrO}_{2}$ formed during post-milling heattreatment was also reduced. Beyond this initial stage, the majority of the hydrated $\mathrm{ZrO}_{2}$ reaction product was formed during milling, thus allowing the formation of aggregates through interparticle welding during collision events. At long milling times, a steadystate particle size was developed presumably as a result of the competing processes of particle aggregation and fracture.

The variation in particles size of the powder formed from the diluted $\mathrm{ZrCl}_{4}+4 \mathrm{LiOH}$ system is in distinct contrast to that exhibited by the $\mathrm{ZrCl}_{4}+2 \mathrm{MgO}$ system where the particle size decreased with milling time down to a lower limit, which indicates that aggregation of the particles did not occur to any significant extent. This difference in behaviour can most likely be attributed to the presence of incipient water, which would have promoted aggregation. A study by Murase and Kato [102] found that water increases the mobility of $\mathrm{ZrO}_{2}$ at crystal surfaces, which facilitates particle growth and sintering.

The formation and compaction of particle aggregates during extended milling of both the diluted and undiluted $\mathrm{ZrCl}_{4}+4 \mathrm{LiOH}$ systems is consistent with previous studies of the effect of mechanical processing on the properties of hydrated oxides. For example, Isobe and Senna [103] found that mechanical activation of hydrated $\mathrm{ZrO}_{2}$ and $\mathrm{TiO}_{2}$ gels resulted in the loss of specific surface area. In the case of $\mathrm{ZrO}_{2}$, vibratory milling for 0.5 hours decreased the specific surface area from 177 to $24.5 \mathrm{~m}^{2} / \mathrm{g}$. Similar results have also been obtained by Kuznetsov et al. [104] in their study of the effects of planetary milling on the physical characteristics of hydrated $\mathrm{ZrO}_{2}$ powder. The particle size, as estimated from the specific surface area, increased from 39 to $51 \mathrm{~nm}$ on milling for 5 minutes before decreasing slightly to $49 \mathrm{~nm}$ by 15 minutes. 


\section{(6.5) Particle Morphology}

For $\mathrm{ZrO}_{2}$ powders synthesised using either $\mathrm{MgO}$ or $\mathrm{Li}_{2} \mathrm{O}$ as the exchange reagent, postreaction heat-treatment at temperatures above the melting point of the by-product salt was found to result in anisotropic particle growth, which ultimately resulted in the formation of $\mathrm{ZrO}_{2}$ particles with a rod-like morphology.

As discussed in Chapter 2, the heat-treatment of oxide precursors in molten salt has previously found widespread use for the synthesis of powders with anisotropic particle shapes. However, the temperatures used have typically been significantly higher than those used in the present study. The successful formation of anisotropic particles despite the low processing temperature is readily attributable to the nanocrystalline size of the $\mathrm{ZrO}_{2}$ particles, which would have increased both the kinetics of dissolution into the salt flux and the thermodynamic driving force for crystal growth.

Particles prepared from $\mathrm{ZrCl}_{4}+2 \mathrm{MgO}$ reactant mixtures were found to exhibit a significantly higher aspect ratio than those prepared from $\mathrm{ZrCl}_{4}+2 \mathrm{Li}_{2} \mathrm{O}+\mathrm{xLiCl}$. This is most likely due to the significantly smaller average particle size of the $\mathrm{ZrO}_{2}$ particles formed by the $\mathrm{ZrCl}_{4}+\mathrm{MgO}$ reaction system. However, it is also possible that the higher aspect ratio is due to a difference in the solubility of $\mathrm{ZrO}_{2}$ in molten $\mathrm{MgCl}_{2}$ as compared to $\mathrm{LiCl}$. This would lead to a changed aspect ratio as the relative growth rates of the various crystal faces decreases with higher levels of supersaturation.

The rod-like growth habit exhibited by the $\mathrm{ZrO}_{2}$ particles in this study is consistent with the results of previous investigations into the synthesis of $\mathrm{ZrO}_{2}$ powders through molten salt processing and hydrothermal treatments. Afanasiev [105] synthesised monodisperse particles of $\mathrm{ZrO}_{2}-\mathrm{CeO}_{2}$ with a rod-like morphology through reaction of $\mathrm{Ce}\left(\mathrm{NO}_{3}\right)_{3}$ and $\mathrm{ZrOCl}_{2}$ in molten $\mathrm{NaNO}_{3}$. Li et al. [106], Ardizzone et al. [107], and Mottet et al. [108] found that rod-like particle of $\mathrm{ZrO}_{2}$ formed during hydrothermal treatment of acidic solutions that contained $\mathrm{Zr}^{4+}$ ions. 


\section{(6.6) Phase Formation and Stability}

\section{(6.6.1) Magnesium Oxide Reactions}

Reaction of $\mathrm{ZrCl}_{4}+2 \mathrm{MgO}$ was found to result in the formation of $\mathrm{ZrO}_{2}$ particles with the tetragonal structure irrespective of the milling duration or dilution. Transformation to the expected monoclinic structure only occurred on heat treatment at temperatures above the melting point of the $\mathrm{MgCl}_{2}$ salt by-product, which caused significant particle growth. This behaviour appears consistent with the concept of a critical particle size below which the tetragonal structure is stabilised for a given temperature [81-84].

\section{(6.6.2) Lithium Oxide Reactions}

For the $\mathrm{ZrCl}_{4}+2 \mathrm{Li}_{2} \mathrm{O}$ reaction system, the decrease in particle and crystallite size with increased dilution was accompanied by an increase in the volume fraction of the tetragonal phase. The undiluted reactant mixture yielded a powder consisting solely of the monoclinic polymorph. Dilution with $2 \mathrm{LiCl}$ resulted in the formation of a $\mathrm{ZrO}_{2}$ powder containing both the monoclinic and tetragonal polymorphs. The tetragonal volume fraction monotonically increased with higher levels of dilution.

The adiabatic reaction temperature of the undiluted $\mathrm{ZrCl}_{4}+2 \mathrm{Li}_{2} \mathrm{O}$ reactant mixture is approximately $1383^{\circ} \mathrm{C}$, which is well in excess of the monoclinic to tetragonal transformation temperature for coarse-grained $\mathrm{ZrO}_{2}$. As a consequence, it would be expected that the $\mathrm{ZrO}_{2}$ product phase would nucleate with the tetragonal structure. However, this tetragonal $\mathrm{ZrO}_{2}$ would then undergo transformation to the equilibrium monoclinic structure on cooling.

According to the surface energy arguments advanced by Garvie, refinement of the $\mathrm{ZrO}_{2}$ particle size through the addition of diluent would have had the effect of lowering the monoclinic to tetragonal transformation temperature [81-83]. It is therefore likely that the $\mathrm{ZrO}_{2}$ product phases formed by the diluted reactant mixtures were also nucleated with the tetragonal structure. Particles with a sufficiently small diameter were able to retain this structure on subsequent cooling whereas the larger particles transformed to the monoclinic form. 
In an effort to derive an estimate of the critical particle diameter, the particle size distribution of the $\mathrm{ZrO}_{2}$ powder synthesised from $\mathrm{ZrCl}_{4}+2 \mathrm{Li}_{2} \mathrm{O}+4 \mathrm{LiCl}$ reactant mixture was measured by TEM. The results are shown in Figure 6.4 as a plot of cumulative volume fraction versus particle diameter. From this plot it can be seen that the $61 \%$ tetragonal volume fraction of this powder corresponds to a critical particle diameter of approximately $32 \mathrm{~nm}$.

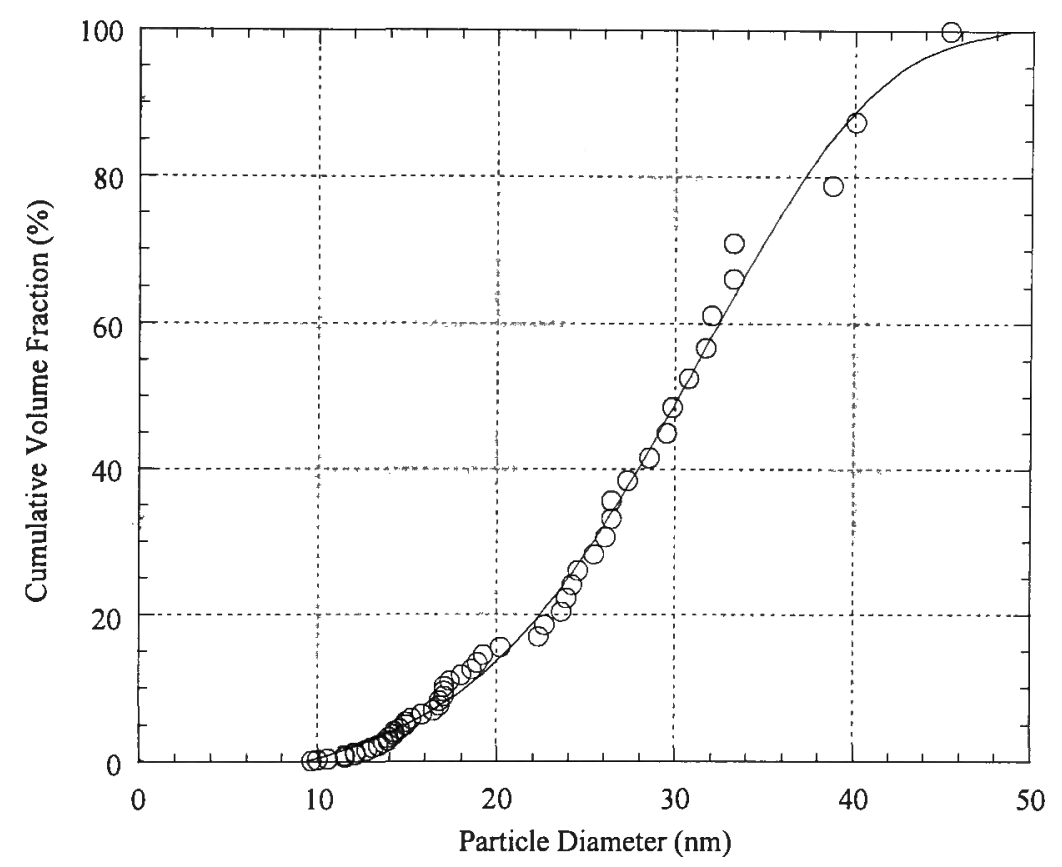

Figure 6.4 : Cumulative volume fraction as a function of particle diameter for $\mathrm{ZrO}_{2}$ powder synthesised from $\mathrm{ZrCl}_{4}+2 \mathrm{Li}_{2} \mathrm{O}+4 \mathrm{LiCl}$.

The behaviour observed in the present study is in contrast to the results obtained by Gillan and Kaner [97] in their study of the $\mathrm{ZrCl}_{4}+2 \mathrm{Na}_{2} \mathrm{O}$ reaction system. In the absence of any diluent, this reaction system was found to result in the formation of a $\mathrm{ZrO}_{2}$ powder with a tetragonal volume fraction of $42 \%$. Dilution with $6 \mathrm{NaCl}$ decreased the tetragonal volume fraction of the resultant $\mathrm{ZrO}_{2}$ powder to less than $19 \%$.

Similar to the $\mathrm{ZrCl}_{4}+2 \mathrm{Li}_{2} \mathrm{O}$ system, the adiabatic reaction temperature of $\mathrm{ZrCl}_{4}+$ $2 \mathrm{Na}_{2} \mathrm{O}$ is in excess of the monoclinic to tetragonal transformation temperature for coarse-grained $\mathrm{ZrO}_{2}$. The $\mathrm{ZrO}_{2}$ product phase would therefore be expected to form with the tetragonal structure. Partial retention of this tetragonal structure on cooling could 
possibly be explained by the presence of domain boundary stresses, which would tend to inhibit complete transformation.

For a given level of dilution, the tetragonal volume fraction was found to be higher for the stabilised zirconia powders than the undoped $\mathrm{ZrO}_{2}$. This readily attributable to the presence of stabilising cations and their smaller average particle size.

\section{(6.6.3) Lithium Hydroxide Reactions}

Combustive reaction of the undiluted $\mathrm{ZrCl}_{4}+4 \mathrm{LiOH}$ reactant mixture was found to result in the formation of a nanocrystalline $\mathrm{ZrO}_{2}$ powder with the high-temperature tetragonal structure. However, continued milling after the combustion event resulted in the progressive transformation to the equilibrium monoclinic structure. This behaviour is consistent with the results of previous studies regarding the effect of mechanical processing on the phase content of tetragonal $\mathrm{ZrO}_{2}$ powders. For example, Bailey et al. [109] found that vibratory milling of metastable tetragonal $\mathrm{ZrO}_{2}$ resulted in rapid transformation to the monoclinic form.

Continued milling of the undiluted reaction systems for the synthesis of stabilised zirconia powders was also found to result in a progressive transformation to the equilibrium monoclinic form. However, in contrast to the $\mathrm{ZrCl}_{4}+4 \mathrm{LiOH}$ system, it was found that the tetragonal structure could be recovered by post-milling heat treatment. This is attributable to the gradual incorporation of the stabilising oxide into the $\mathrm{ZrO}_{2}$ with progressively higher heat treatment temperatures.

Gradual reaction of the diluted reactant mixture resulted in the formation of an amorphous $\mathrm{ZrO}_{2}$ gel that crystallized with the tetragonal structure on calcination at $500^{\circ} \mathrm{C}$ for 1 hour. The crystallization of tetragonal $\mathrm{ZrO}_{2}$ from amorphous gels has been attributed to structural similarities between the amorphous and tetragonal structures [86]. 


\section{Chapter 7}

\section{Conclusions and Suggestions for Further Work}

\section{(7.1) Summary and Conclusions}

All of the systems that were investigated in this study involved the reaction of anhydrous chloride precursors with an oxide exchange reagent. However, despite this fundamental similarity, the systems were found to exhibit significantly different chemical evolution during processing. Depending on the exchange reagent used and the presence of inert diluent, reaction during processing was found to occur either gradually during milling, by mechanically activated combustion, or only during post-milling heat treatment.

The results obtained in this study show that the mechanism of reaction for a given system is determined by both thermodynamic and kinetic factors. All of the reaction systems that were studied are characterised by a negative free energy change, which indicates that chemical reaction is thermodynamically favourable and could therefore be expected to occur during milling. However, for systems that used $\mathrm{Li}_{2} \mathrm{O}$ as the exchange reagent, chemical reaction was only found to occur during post-milling heat treatment. In contrast, all other systems based on $\mathrm{MgO}$ or $\mathrm{LiOH}$ were found to undergo chemical reaction during milling.

For anhydrous reaction systems, the adiabatic reaction temperature associated with formation of the product phases, which is a function of the enthalpy change and diluent concentration, has been shown to be an important factor in determining the mechanism of chemical reaction. Specifically, a sufficiently high adiabatic reaction temperature is required for the enthalpy change to influence the reaction kinetics.

In the case of the $\mathrm{ZrCl}_{4}+2 \mathrm{MgO}+\mathrm{xMgCl}_{2}$ system, the adiabatic reaction temperature was low for all diluent concentrations. As a result, the heat generated locally by chemical reaction during collisions of the grinding media was insufficient to alter the overall reaction kinetics. Consequently, reaction occurred in a gradual manner during milling, which allowed ultrafine $\mathrm{ZrO}_{2}$ powders to be synthesised by either milling the 
precursors until complete reaction was achieved or by milling for a short duration and then driving the reaction to completion by low temperature heat treatment.

The $\mathrm{ZrCl}_{4}+2 \mathrm{Li}_{2} \mathrm{O}$ system is characterised by a high adiabatic reaction system. Accordingly, the as-milled reactant mixture was found to undergo chemical reaction in a combustive manner during post-milling heat treatment. Thermal analysis of the undiluted $\mathrm{ZrCl}_{4}+2 \mathrm{Li}_{2} \mathrm{O}$ system showed that the temperature required for the initiation of combustive reaction is significantly lower than that observed for comparable solidstate metathesis reaction systems due to the effects of mechanical activation. The addition of $\mathrm{LiCl}$ as an inert diluent raised the initiation temperature to the melting point of the $\mathrm{LiCl}-\mathrm{ZrCl}_{4}$ eutectic, which is consistent with previous studies that have shown that the onset of melting accelerates the kinetics of chemical reaction.

The reaction behaviour of the undiluted $\mathrm{ZrCl}_{4}+4 \mathrm{LiOH}$ system showed that the magnitude of the adiabatic reaction temperature is not sufficient to predict the reaction mechanism in hydrated systems. Reaction of $\mathrm{ZrCl}_{4}+4 \mathrm{LiOH}$ to form $\mathrm{ZrO}_{2}, \mathrm{LiCl}$, and $\mathrm{H}_{2} \mathrm{O}_{(\mathrm{g})}$ is characterised by an adiabatic temperature of only $616^{\circ} \mathrm{C}$, which is significantly lower than the temperatures typically associated with mechanochemical combustion reactions. The occurrence of combustive reaction despite the low adiabatic reaction clearly demonstrates the effect of incipient water on the kinetics of mechanochemical reactions. Evidently, the evolution of water vapour during chemical reaction of the precursors facilitates the propagation of reaction to neighbouring volume elements in the powder charge.

In addition to affecting the mechanism of chemical reaction during processing, the adiabatic reaction temperature was also found to be significant factor in determining the average particle and crystallite size of the final washed powder. Previous studies have shown that the particle size of the product formed during heat treatment could be directly related to the microstructural scale of the as-milled structure. However, such an analysis assumes that no significant particle growth occurs during reaction. The results obtained in the present study of the $\mathrm{ZrCl}_{4}+2 \mathrm{Li}_{2} \mathrm{O}+\mathrm{xLiCl}$ system show that this assumption is invalid for reaction systems that are characterised by a high adiabatic reaction temperature. 
In contrast to the $\mathrm{ZrCl}_{4}+2 \mathrm{Li}_{2} \mathrm{O}+\mathrm{xLiCl}$ system, the adiabatic reaction temperature of the $\mathrm{ZrCl}_{4}+2 \mathrm{MgO}+\mathrm{xMgCl}_{2}$ system is low for all levels of dilution. As a result, the heat released during reaction would have been insufficient to cause any significant particle growth. The average particle size of the final $\mathrm{ZrO}_{2}$ powder was therefore determined solely by the scale of the reactant microstructure, which is consistent with the experimentally observed decrease in particle size with milling time.

The observed variation in particle size with milling time displayed by the $\mathrm{ZrCl}_{4}+$ $4 \mathrm{LiOH}+\mathrm{xLiCl}$ system demonstrated that incipient water promotes the aggregation of $\mathrm{ZrO}_{2}$ particles during milling. As a result, the particle size and degree of agglomeration of the $\mathrm{ZrO}_{2}$ product was found to depend strongly on the milling duration. For the undiluted reaction system, continued milling of the powder charge after the combustion event resulted in a progressive loss of specific surface area, which was due to compaction of the particle agglomerates. The diluted reaction system showed an initial decrease in particle size as a consequence of increased microstructural refinement of the reactants. Further milling resulted in aggregation of the particles before the attainment of a steady-state particle size.

\section{(7.2) Suggestions for Further Work}

The basic reaction scheme investigated in this study, involving reaction of anhydrous chloride precursors with an oxide exchange reagent, appears applicable to the synthesis of a wide variety of other technologically important oxide ceramics. In particular, the reaction systems based on alkali metal oxides and hydroxides could be used to manufacture rare earth oxide powders, which find use in a wide variety of advanced engineering applications.

The results obtained in the present study indicate that a reexamination of the $\mathrm{ZrCl}_{4}+$ $2 \mathrm{CaO}$ reaction system is warranted. In their original study, Ding et al. [29] examined only the undiluted system with a limited number of experimental variables. The behaviour of the $\mathrm{ZrCl}_{4}+2 \mathrm{Li}_{2} \mathrm{O}+\mathrm{xLiCl}$ system suggests that the milling time, dilution, and heating rate could have an important effect on the properties of $\mathrm{ZrO}_{2}$ synthesised from $\mathrm{ZrCl}_{4}+2 \mathrm{CaO}$. 
Mottet et al. [108] showed that the morphology of $\mathrm{ZrO}_{2}$ particles formed by hydrothermal processing is strongly affected by the use of additives. By use of different mineralisers, $\mathrm{ZrO}_{2}$ particles with equiaxed, platelike, and elongated shapes were prepared. This suggests that ultrafine $\mathrm{ZrO}_{2}$ powders with a variety of particle shapes could successfully be synthesised by mechanochemical processing through the use of molten salt processing with suitable additives. 


\section{References}

[1] H. Gleiter, "Nanocrystalline Materials", Progress in Materials Science, Vol. 30 (1989), p 223.

[2] H. Hahn, J. Logas, and R.S. Averback, "Sintering Characteristics of Nanocrystalline $\mathrm{TiO}_{2}$ ", Journal of Materials Research, Vol. 5 (1990), p 609.

[3] H. Hahn and R.S. Averback, "Low-Temperature Creep of Nanocrystalline Titanium[IV] Oxide", Journal of the American Ceramic Society, Vol. 74 (1991), p 2918.

[4] G. Skandan, "Processing of Nanostructured Zirconia Ceramics", Nanostructured Materials, Vol. 5 (1999), p 111.

[5] M.J. Mayo, D.C. Hague, and D.-J. Chen, "Processing Nanocrystalline Ceramics for Applications in Superplasticity", Materials Science and Engineering A, Vol. 166 (1993), p 145.

[6] M.J. Readey, R-R. Lee, J.W. Halloran, and A.H. Heur, "Processing and Sintering of Ultrafine $\mathrm{MgO}-\mathrm{ZrO}_{2}$ and $\left(\mathrm{MgO}, \mathrm{Y}_{2} \mathrm{O}_{3}\right)-\mathrm{ZrO}_{2}$ Powders", Journal of the American Ceramic Society, Vol. 73 (1990), p 1499.

[7] S.G. Malghan, "Comminution", Engineered Materials Handbook, Vol. 4 (1991), p 75.

[8] D. Ganguli and M. Chatterjee, "Ceramic Powder Preparation: A Handbook", Kluwer Academic Publishers (1997).

[9] R.W. Siegel, "Cluster-Assembled Nanophase Materials", Annual Review of Materials Science, Vol. 21 (1991), p 559.

[10] H. Schmalzried, "Chemical Kinetics of Solids", VCH (1995). 
[11] A. Stein, S.W. Keller, and T.E. Mallouk, "Turning Down the Heat: Design and Mechanism in Solid-State Synthesis", Science, Vol. 259 (1993), p 1558.

[12] P.G. McCormick, "Application of Mechanical Alloying to Chemical Refining", Materials Transactions JIM, Vol. 36 (1995), p 161.

[13] P.G. McCormick and F.H. Froes, "The Fundamentals of Mechanochemical Processing", JOM, Vol. 50 (1998), p 61.

[14] C.C. Koch, "Synthesis of Nanostructured Materials by Mechanical Milling: Problems and Opportunities", Nanostructured Materials, Vol. 9 (1997), p 13.

[15] P.G. McCormick, "Mechanical Alloying and Mechanically Induced Chemical Reactions", Handbook on the Physics and Chemistry of Rare Earths, Vol. 24 (1997), p 47, Elsevier Science.

[16] C. Suryanarayana, "Mechanical Alloying and Milling", Progress in Materials Science, Vol. 46 (2001), p 1.

[17] P.G. McCormick, Y. Liu, H. Yang, G. Nguyen, and T. Alonso, "Chemical Refining Via Mechanical Alloying", Proceedings of the 2nd International Conference on Structural Applications of Mechanical Alloying (1993), p 165.

[18] M. Dallimore, H. Huang, J. Ding, J. Pan, and P.G. McCormick, Proceedings of the 2nd International Conference on Structural Applications of Mechanical Alloying (1993), p 45.

[19] G.B. Schaffer and P.G. McCormick, "The Direct Synthesis of Metals and Alloys by Mechanical Alloying”, Materials Science Forum, Vol. 88-90 (1992), p 779.

[20] H. Yang and P.G. McCormick, "Combustion Reaction of Zinc Oxide with Magnesium During Mechanical Milling", Journal of Solid State Chemistry, Vol. 107 (1993), p 258. 
[21] G.B. Schaffer and P.G. McCormick, "On the Kinetics of Mechanical Alloying", Metallurgical Transactions A, Vol. 23 (1992), p 1285.

[22] L. Takacs, V. Soika, and P. Balaz, "The effect of Mechanical Activation on Highly Exothermic Powder Mixtures", Solid State Ionics, Vol. 141-142 (2001), p 641 .

[23] H. Yang and P.G. McCormick, "Mechanochemical Reduction of $\mathrm{V}_{2} \mathrm{O}_{5}$ ", Journal of Solid State Chemistry, Vol. 110 (1994), p 136.

[24] M. Atzmon, "In Situ Thermal Observation of Explosive Compound Formation Reaction During Mechanical Alloying", Physical Review Letters, Vol. 64 (1990), p 487.

[25] G.B. Schaffer and P.G. McCormick, "Reduction of Metal Oxides by Mechanical Alloying”, Applied Physics Letters, Vol. 55 (1989), p 45.

[26] R.M. Ren, Z.G. Yang, and L.L. Shaw, "Synthesis of Nanostructured TiC via Carbothermic Reduction Enhanced by Mechanical Activation", Scripta Materialia, Vol. 38 (1998), p 735.

[27] J. Ding, T. Tsuzuki, and P.G. McCormick, "Hematite Powders Synthesised by Mechanochemical Processing”, Nanostructured Materials, Vol. 8 (1997), p 739.

[28] J. Ding, T. Tsuzuki, and P.G. McCormick, "Ultrafine Alumina Particles Prepared by Mechanochemical/Thermal Processing", Journal of the American Ceramic Society, Vol. 79 (1996), p 2958.

[29] J. Ding, T. Tsuzuki, and P.G. McCormick, "Mechanochemical Synthesis of Ultrafine $\mathrm{ZrO}_{2}$ Powder", Nanostructured Materials, Vol. 8 (1997), p 75.

[30] C. Gras, E. Gaffet, F. Bernard, F. Charlot, and J.C. Niepce "Mechanically Activated Self-Propagating High Temperature Synthesis (MASHS) Applied to 
$\mathrm{MoSi}_{2}$ and $\mathrm{FeSi}_{2}$ Phase Formation", Materials Science Forum, Vol. 312-314 (1999), p 281.

[31] J. Ding, T. Tsuzuki, and P.G. McCormick, "Microstructural Evolution of Ni$\mathrm{NaCl}$ Mixtures during Mechanochemical Reaction and Mechanical Milling”, Journal of Materials Science, Vol. 34 (1999), p 5293.

[32] J. Ding, T. Tsuzuki, and P.G. McCormick, "Structure and Magnetic Properties of Ultrafine Fe Powders by Mechanochemical Processing", Journal of Magnetism and Magnetic Materials, Vol. 162 (1996), p 271.

[33] J. Ding, T. Tsuzuki, P.G. McCormick, and R. Street, "Ultrafine Co and Ni Particles Prepared by Mechanochemical Processing", Journal of Physics D, Vol. 29 (1996), p 2365.

[34] J. Ding, T. Tsuzuki, and P.G. McCormick, "Ultrafine Cu Particles Prepared by Mechanochemical Processing”, Journal of Alloys and Compounds, Vol. 234 (1996), p L1.

[35] N.Q. Wu, L.Z. Su, M.Y. Yuan, J.M. Wu, and Z.Z. Li, "Nano-Sized Amorphous $\mathrm{Cu}-\mathrm{Zr}$ Alloy Particles Prepared by Mechanochemical Processing", Materials Science and Engineering A, Vol. 257 (1998), p 357.

[36] W. Liu and P.G. McCormick, "Synthesis of $\mathrm{Sm}_{2} \mathrm{Co}_{17}$ Alloy Nanoparticles by Mechanochemical Processing", Journal of Magnetism and Magnetic Materials, Vol. 195 (1999), p L279.

[37] N.J. Welham and D.J. Llewellyn, "Formation of Nanometric Hard Materials by Cold Milling”, Journal of the European Ceramic Society, Vol. 19 (1999), p 2833.

[38] T. Tsuzuki and P.G. McCormick, "Synthesis of $\mathrm{Cr}_{2} \mathrm{O}_{3}$ Nanoparticles by Mechanochemical Processing”, Acta Materialia, Vol 48 (2000), p 2795. 
[39] E.G. Gillan and R.B. Kaner, "Synthesis of Refractory Ceramics via Rapid Metathesis Reactions between Solid-State Precursors", Chemistry of Materials, Vol. 8 (1996), p 333.

[40] M. Muroi, R. Street, P.G. McCormick, and J. Amighian, "Magnetic Properties of Ultrafine $\mathrm{MnFe}_{2} \mathrm{O}_{4}$ Powders Prepared by Mechanochemical Processing", Physical Review B, Vol. 63, p 184414.

[41] T. Tsuzuki, W.T.A. Harrison, and P.G. McCormick, "Synthesis of Ultrafine Gadolinium Oxide Powder by Mechanochemical Processing", Journal of Alloys and Compounds, Vol. 281 (1998), p 146.

[42] T. Tsuzuki, E. Pirault, and P.G. McCormick, "Mechanochemical Synthesis of Gadolinium Oxide Nanoparticles", Nanostructured Materials, Vol. 11 (1999), p 125.

[43] J. Ding, T. Tsuzuki, and P.G. McCormick, "Ultrafine $\mathrm{BaFe}_{12} \mathrm{O}_{19}$ Powder Synthesised by Mechanochemical Processing", Journal of Magnetism and Magnetic Materials, Vol. 177 (1998), p 931.

[44] T. Tsuzuki and P.G. McCormick, "ZnO Nanoparticles Synthesised by Mechanochemical Processing", Scripta Materialia, Vol. 44 (2001), p 1731.

[45] T. Tsuzuki and P.G. McCormick, "Synthesis of Metal-Oxide Nanoparticles by Mechanochemical Processing", Materials Science Forum, Vol. 343-346 (2000), p 383.

[46] L.M. Cukrov, T. Tsuzuki, and P.G. McCormick, " $\mathrm{SnO}_{2}$ Nanoparticles Prepared by Mechanochemical Processing", Scripta Materialia, Vol. 44 (2001), p 1787

[47] T. Tsuzuki and P.G. McCormick, "Synthesis of Ultrafine Ceria Powders by Mechanochemical Processing", Journal of the American Ceramic Society, Vol. 84 (2001), p 1453. 
[48] S. Goplan and S. Singhal, "Mechanochemical Synthesis of Nano-Size $\mathrm{CeO}_{2}$ ", Scripta Materialia, Vol. 42 (2000), p 993.

[49] T. Tsuzuki, J. Ding, and P.G. McCormick, "Mechanochemical Synthesis of Ultrafine Zinc Sulfide Particles", Physica B, Vol. 239 (1997), p 378.

[50] T. Tsuzuki and P.G. McCormick, "Synthesis CdS Quantum Dots by Mechanochemical Reaction”, Applied Physics A, Vol. 65 (1997), p 607.

[51] T. Tsuzuki and P.G. McCormick, "Mechanochemical Synthesis of Metal Sulphide Nanoparticles", Nanostructured Materials, Vol. 12 (1999), p 75.

[52] S. Cai, T. Tsuzuki, T. Fisher, and P.G. McCormick, "Synthesis of Nanocrystalline Gallium Nitride by Mechanochemical Reaction", Conference on Optoelectronic and Microelectronic Materials and Devices, New Jersey, USA, Institute of Electrical and Electronic Engineers (1999), p 400.

[53] X. Liu, J. Ding, and J. Wang, "An $\alpha-\mathrm{Fe}_{2} \mathrm{O}_{3}$ Powder of Nanosized Particles via Precursor Dispersion", Journal of Materials Research, Vol. 14, p 3355.

[54] Y. Shi, J. Ding, and H. Yin, " $\mathrm{CoFe}_{2} \mathrm{O}_{4}$ Nanoparticles Prepared by the Mechanochemical Method", Journal of Alloys and Compounds, Vol. 308 (2000), p 290.

[55] Y. Shi, J. Ding, X. Liu, and J. Wang, " $\mathrm{NiFe}_{2} \mathrm{O}_{4}$ Ultrafine Particles Prepared by Co-Precipitation/Mechanical Alloying", Journal of Magnetism and Magnetic Materials, Vol. 205 (1999), p 249.

[56] C.P. Fah, J. Xue, and J. Wang, "Nanosized Zinc-Oxide Particles Derived from Mechanical Activation of $\mathrm{Zn}_{5}\left(\mathrm{NO}_{3}\right)_{2}(\mathrm{OH})_{8} \cdot 2 \mathrm{H}_{2} \mathrm{O}$ in Sodium Chloride", Journal of the American Ceramic Society, Vol. 85 (2002), p 273.

[57] N.J. Welham, "Formation and Characterisation of Germanium Nanoparticles", Journal of Materials Research, Vol. 15 (2000), p 2400. 
[58] D. Stauffer and A. Aharony, "Introduction to Percolation Theory", Taylor and Francis Ltd. (1994).

[59] S. Hashimoto and A. Yamaguchi, "Synthesis of Needlelike Mullite Particles using Potassium Sulfate Flux", Journal of the European Ceramic Society, Vol. 20 (2000), p 397.

[60] T. Kimura, T. Kanazawa, and T. Yamaguchi, "Preparation of $\mathrm{Bi}_{4} \mathrm{Ti}_{3} \mathrm{O}_{12}$ Powders in the Presence of Molten Salt Containing LiCl", Journal of the American Ceramic Society, Vol. 66 (1983), p 597.

[61] A.C. Tas, "Molten Salt Synthesis of Calcium Hydroxyapatite Whiskers", Journal of the American Ceramic Society, Vol. 84 (2001), p 295.

[62] S. Hayashi, M. Sugai, Z-e. Nakagawa, T. Takei, K. Kawasaki, T. Katsuyama, A. Yasumori, and K. Okada, "Preparation of $\mathrm{CaSiO}_{3}$ Whiskers from Alkali Halide Fluxes", Journal of the European Ceramic Society, Vol. 20 (2000), p 1099.

[63] S. Oishi and M. Hirao, "Growth of $\mathrm{CaWO}_{4}$ Whiskers from $\mathrm{KCl}$ Flux", Journal of Materials Science Letters, Vol. 8 (1989), p 1397.

[64] R.F. Hill, R. Danzer, and R.T. Paine, "Synthesis of Aluminum Platelets", Journal of the American Ceramic Society, Vol. 84 (2001), p 514.

[65] R.H. Arendt, "The Molten Salt Synthesis of Single Magnetic Domain $\mathrm{BaFe}_{12} \mathrm{O}_{19}$ and $\mathrm{SrFe}_{12} \mathrm{O}_{19}$ Crystals", Journal of Solid State Chemistry, Vol. 8 (1973), p 339.

[66] T. Takeuchi, T. Tani, and T. Satoh, "Microcomposite Particles $\mathrm{Sr}_{3} \mathrm{Ti}_{2} \mathrm{O}_{7}-\mathrm{SrTiO}_{3}$ with an Epitaxial Core-Shell Structure", Solid State Ionics, Vol. 108 (1998), p 67.

[67] J-H Choy and Y-S Han, "A Combinative Flux Evaporation-Slow Cooling Route to Potassium Titanate Fibres", Materials Letters, Vol. 34 (1998), p 111. 
[68] B.R. Pamplin, “Crystal Growth”, Pergamon Press (1975).

[69] F. Kawamura, I.Yasui, M. Kamei, and I. Sunagawa, "Habit Modifications of $\mathrm{SnO}_{2}$ Crystals in $\mathrm{SnO}_{2}-\mathrm{Cu}_{2} \mathrm{O}$ Flux System in the Presence of Trivalent Impurity Cations", Journal of the American Ceramic Society, Vol. 84 (2001), p 1341.

[70] "Encyclopedia of Chemical Technology", Vol. 25 (1998), John Wiley \& Sons.

[71] W.E. Lee and W.M. Rainforth, "Ceramic Microstructures: Property Control by Processing", Chapman Hall (1994).

[72] A.H. Heuer, "Transoformation Toughening in $\mathrm{ZrO}_{2}$-Containing Ceramics", Journal of the American Ceramic Society", Vol. 70 (1987), p 689.

[73] I. Birkby and R. Stevens, "Applications of Zirconia Ceramics", Key Engineering Materials, Vol. 122 (1996), p 527.

[74] T. Yamaguchi and J. Hightower, "Hydrogenation of 1,3-Butadiene with 1,3Cyclohexadiene and Molecular Deuterium Over Zirconium Dioxide Catalysts", Journal of the American Chemical Society, Vol. 99 (1977), p 4201.

[75] Y. Nakano, H. Iizuka, H. Hattori, and K. Tanabe, "Surface Properties of Zirconium Oxide and its Catalytic Activity for Isomerization of 1-Butene", Journal of Catalysis, Vol. 57 (1979), p 1.

[76] T. Yamaguchi, H. Sasaki, and K. Tanabe, "High Selectivity of a Zirconium Dioixde Catalyst for the Isomerization of 1-Butene and Dehydration of SecButanol", Chemistry Letters (1973), p 1017.

[77] L. Bruce and J.F. Mathews, "The Fischer-Tropsch Activity of Nickel-Zirconia", Applied Catalysis, Vol. 4 (1982), p 353. 
[78] Y. Amenomiya, "Methanol Synthesis from Carbon Dioxide + Hydrogen. II. Copper Based Binaryy and Ternary Catalysts", Applied Catalysis, Vol. 30 (1987), p 57.

[79] D. Hamon, M. Vrinat, M. Breysse, B. Durand, M. Jebrouni, M. Roubin, P. Magnoux, T. des Courieres, "Molten Salt Preparation of Stabilized Zirconia Catalysts: Characterization and Catalytic Properties", Catalysis Today, Vol. 10 (1991), p 613.

[80] E.H. Kisi and C.J. Howard, "Crystal Structures of Zirconia and their InterRelation", Key Engineering Materials, Vol. 153-154 (1998), p 1.

[81] R.C. Garvie, "The Occurrence of Metastable Tetragonal Zirconia as a Crystallite Size Effect”, Journal of Physical Chemistry, Vol. 69 (1965), p 1238.

[82] R.C. Garvie, "Stabilization of the Tetragonal Structure in Zirconia Microcrystals", Physical Chemistry, Vol. 82 (1978), p 218.

[83] R.C. Garvie and M.F. Goss, "Intrinsic Size Dependence of the Phase Transformation Temperature in Zirconia Microcrystals", Journal of Materials Science, Vol. X (1986), p 1253.

[84] N-L. Wu, T-F Wu, and I.R. Rusakova, "Thermodynamic Stability of Tetragonal Zirconia Nanocrystallites", Journal of Materials Research, Vol. 16 (2001), p 666.

[85] D.E. Collins and K.J. Bowman, "Influence of Atmosphere on Crystallization of Zirconia From a Zirconium Alkoxide", Journal of Materials Research, Vol. 13 (1998), p 1230.

[86] J. Livage, K. Doi, and C. Mazieres, "Nature and Thermal Evolution of Amorphous Hydrated Zirconium Oxide", Journal of the American Ceramic Society, Vol. 51 (1968), p 349. 
[87] S. Xie, E. Iglesia, and A.T. Bell, "Water-Assisted Tetragonal-to-Monoclinic Phase Transformation of $\mathrm{ZrO}_{2}$ at Low Temperatures", Chemistry of Materials, Vol. 12 (2000), p 2442.

[88] B. Bachiller-Baeza, I. Rodriguez-Ramos, and A. Guerrero-Ruiz, "Interaction of Carbon Dioxide with the Surface of Zirconia Polymorphs", Langmuir, Vol. 14 (1998), p 3556.

[89] T. Chraska, A.H. King, and C.C. Berndt, "On the Size-Dependent Phase Transformation in Nanoparticulate Zirconia", Materials Science and Engineering, Vol. A286 (2000), p 169.

[90] http://www.geol.uni-erlangen.de/html/software/winsoft.html

[91] H. Toraya, M. Yoshimura, and S. Somiya, "Quantitative Analysis of Monoclinic-Stabilized Cubic $\mathrm{ZrO}_{2}$ Systems by X-Ray Diffraction”, Vol. 67 (1984), p C-183.

[92] H. Toraya, M. Yoshimura, and S. Somiya, "Calibration Curve for Quantitative Analysis of the Monoclinic-Tetragonal $\mathrm{ZrO}_{2}$ System by X-Ray Diffraction", Journal of the American Ceramic Society, Vol. 67 (1984), p C-119.

[93] B. Cullity, "Elements of X-Ray Diffraction", $2^{\text {nd }}$ Edition. Addison-Wesley, Reading (1978).

[94] J.J. Moore and H.J. Feng, "Combustion Synthesis of Advanced Materials: Part I. Reaction Parameters", Progress in Materials Science, Vol. 39 (1995), p 243.

[95] A.L. Hector, G. Henshaw, A.V. Komarov, and I.P. Parkin, "Nitrides from Solid State Metathesis Reactions: Synthesis and mechanistics", Journal of Materials Processing Technology, Vol. 77 (1998), p 103. 
[96] A.L. Hector and I.P. Parkin, "Low Temperature Solid-State Routes to Transition Metal Oxide via Metathesis Reactions Involving Lithium Oxide", Polyhedron, Vol. 12 (1993), p 1855.

[97] E.G. Gillian and R.B. Kaner, "Rapid, Energetic Metathesis Routes to Crystalline Metastable Phases of Zirconium and Hafnium Dioxide," Journal of Materials Chemistry, Vol. 11 (2001), p 1951.

[98] Y. Song, M. Gui, T. Zheng, Y. Zhang, and Z. Liu, "Phase Diagram of the Zirconium Chloride-Lithium Chloride System", Xiyou Jinshu, Vol. 2 (1983), p 75.

[99] M. J. Mayo, J. R. Seidensticker, D. C. Hague and A. H. Carim, "Surface Chemistry Effects on the Processing and Superplastic Properties of Nanocrystalline Oxide Ceramics", Nanostructured Materials, Vol. 11 (1999), p 271.

[100] N.J. Welham, and D.J. Llewellyn, "Mechanical Enhancement of the Dissolution of Ilmenite", Minerals Engineering, Vol. 11 (1998), p 827.

[101] J.B. Wiley and R.B. Kaner, "Rapid Solid-State Precursor Synthesis of Minerals", Science, Vol. 255 (1992), p 1093.

[102] Y. Murase and E. Kato, "Role of Water Vapor in Crystallite Growth and Tetragonal-Monoclinic Phase Transformation of Zirconia", Journal of the American Ceramic Society, Vol. 66 (1983), p 196.

[103] T. Isobe and M. Senna, "Effects of Mechanical Activation on Inorganic Gels on Their Thermal Behavior. 2. Comparison of Structure and Thermal Behavior between Zirconia and Titania Gels", Journal of Solid State Chemistry, Vol. 93 (1991), p 368.

[104] P.N. Kuznetsov, L.I. Kuznetsova, A.M. Zhyzhaev, G.L. Pashkov, and V.V. Boldyrev, "Ultra Fast Synthesis of Metastable Tetragonal Zirconia by Means of 
Mechanochemical Activation", Applied Catalysis A: General, Vol 227 (2002), p 299.

[105] P. Afanasiev, "Ce-Zr Mixed Oxides Prepared in Molten Nitrates", Journal of Alloys and Compounds, Vol. 340 (2002), p 74.

[106] C. Li, I. Wamai, Y. Murase, and E. Kato, "Formation of Acicular Monoclinic Zirconia Particles Under Hydrothermal Conditions", Journal of the American Ceramic Society, Vol. 72 (1989), p 1479.

[107] S. Ardizzone, P. Lazzari, and E. Sivieri, "Temperature Effects on Rod_like Zirconia Particles. Morphology, Degree of Crystallinity and Texture", Materials Letters, Vol. 16 (1993), p 45.

[108] B. Mottet, M. Pichavant, J-M. Beny, and J-A. Alary, "Morphology of Zirconia Synthesized from Zirconium Oxychloride", Journal of the American Ceramic Society, Vol. 75 (1992), p 2515.

[109] J.E. Bailey, D. Lewis, Z.M. Librant, and L.J. Porter, "Phase Transformations in Milled Zirconia", Transactions of the Journal of the British Ceramic Society, Vol. 71 (1972), p 25.

[110] M.S. Kaliszewski and A.H. Heur, "Alcohol Interaction with Zirconia Powders", Journal of the American Ceramic, Vol. 73 (1990), p 1504. 


\section{Publications During PhD Candidature}

A.C. Dodd and P.G. McCormick, "Synthesis and Processing of an Ultrafine Mg-PSZ Powder", Materials Science Forum, Vol. 132-134 (1999), p 221.

A.C. Dodd and P.G. McCormick, "Mechanochemical Synthesis of Nano Zirconia Powders", Journal of the Australasian Ceramic Society, Vol. 36 (2000), p 15.

A.C. Dodd and P.G. McCormick, "Solid-State Chemical Synthesis of Nanoparticulate Zirconia", Acta Materialia, Vol. 49 (2001), p 4215.

A.C. Dodd, K. Raviprasad, and P.G. McCormick, "Synthesis of Ultrafine Zirconia Powders by Mechanochemical Processing", Scripta Materialia, Vol. 44 (2001), p 689.

A.C. Dodd and P.G. McCormick, "Synthesis of Nanoparticulate Zirconia by Mechanochemical Processing", Scripta Materialia, Vol. 44 (2001), p 1725.

A.C. Dodd and P.G. McCormick, "Synthesis of Nanocrystalline $\mathrm{ZrO}_{2}$ Powders by Mechanochemical Reaction of $\mathrm{ZrCl}_{4}$ with $\mathrm{LiOH}$ ", Journal of the European Ceramic Society, Vol. 22 (2002), p 1823.

A.C. Dodd and P.G. McCormick, "Factors Affecting the Particle Size of Powders Synthesised by Mechanochemical Processing, Journal of Metastable and Nanocrystalline Materials. Submitted for Publication (2002). 